COMMUNICATIONS IN

ANALYSIS AND GEOMETRY

Volume 9, Number 1, 169-237, 2001

\title{
Constant mean curvature surfaces with Delaunay ends
}

\author{
Rafe Mazzeo ${ }^{1}$ and Frank Pacard ${ }^{2}$
}

\section{Introduction and statement of the results.}

In this paper we shall present a construction of complete surfaces $M$ in $\mathbb{R}^{3}$ with finitely many ends and finite topology, and with nonzero constant mean curvature (CMC). This construction is parallel to the well-known original construction by Kapouleas [5], but we feel that ours is somewhat simpler analytically, and controls the resulting geometry more closely. On the other hand, the surfaces we construct have a rather different, and usually simpler, geometry than those of Kapouleas; in particular, all of the surfaces constructed here are noncompact, so we do not obtain any of his immersed compact examples. The method we use here closely parallels the one we developed recently [10] to study the very closely related problem of constructing Yamabe metrics on the sphere with $k$ isolated singular points, just as Kapouleas' construction parallels the earlier construction of singular Yamabe metrics by Schoen [18].

The original examples of noncompact CMC surfaces were those in the one-parameter family of rotationally invariant surfaces discovered by Delaunay in 1841 [2]. One extreme element of this family is the cylinder; the 'Delaunay surfaces' are periodic, and the embedded members of this family (which are called unduloids) interpolate between the cylinder and an infinite string of spheres arranged along a common axis. The family continues beyond this, but the elements now are immersed (and are called nodoids).

The rôle of Delaunay surfaces in the theory of complete CMC surfaces is analogous to the rôle of catenoids (and planes) in the study of complete minimal surfaces of finite total curvature. For example, just as any complete minimal surface with two ends must be a catenoid [19], it was proved by

\footnotetext{
${ }^{1}$ Supported by the NSF under Grant DMS-9626382 and a Young Investigator Fellowship, and by a Sloan Foundation Fellowship

${ }^{2}$ Supported by the American Institute of Mathematics
} 
Meeks [14] and Korevaar, Kusner and Solomon [8] that any Alexandrov embedded constant mean curvature surface with at most two ends is necessarily a Delaunay surface. A rather more remarkable theorem, paralleling the fact that any end of a complete minimal surface of finite total curvature must be asymptotic to a catenoid or a plane, is the fact that any embedded end of a CMC surface must be asymptotic to one of these rotationally symmetric Delaunay surfaces (and in particular, must be cylindrically bounded).

The fact that such CMC surfaces exist in abundance was proved, as noted above, by Kapouleas [5] in 1987. Further developments were given in [7]. More recently, using different methods based on the Schwarz reflection principle, Grosse-Brauckmann has constructed families of CMC surfaces with $k$ ends and with $k$-fold dihedral symmetry [3].

We shall denote by $\mathcal{M}_{g, k}$ the moduli space of CMC surfaces with genus $g$ and $k$ ends of Delaunay type. This space decomposes as

$$
\mathcal{M}_{g, k}=\mathcal{M}_{g, k}^{u} \cup \mathcal{M}_{g, k}^{n} \cup \mathcal{M}_{g, k}^{m},
$$

where $\mathcal{M}_{g, k}^{u}$ is the set of those surfaces all of whose ends are asympotic to embedded Delaunay surfaces (unduloids), $\mathcal{M}_{k, g}^{n}$ is the set of those surfaces all of whose ends are asympotic to immersed Delaunay surfaces (nodoids) and $\mathcal{M}_{k, g}^{m}$ is the set of those surfaces having ends of both types.

The general analysis of the moduli space of Alexandrov embedded CMC surfaces was considered by the first author, Kusner and Pollack [9] (essentially merely translating the analogous results in [13] for the singular Yamabe problem). The results there easily translate to the slightly more general moduli space $\mathcal{M}_{g, k}$ (the elements of which are not required to be Alexandrov embedded, but simply have Delaunay ends) without any difficulty. The basic result is that $\mathcal{M}_{g, k}$ is a locally real analytic variety of virtual dimension $3 k$ (before dividing out by the action of the group of Euclidean motions). This virtual dimension is attained at any point $\Sigma \in \mathcal{M}_{g, k}$ where a certain analytic nondegeneracy criterion is satisfied. This condition will be explained in some detail below. It seems very difficult to decide whether any of the surfaces constructed by Kapouleas satisfy this nondegeneracy criterion. This was one motivation for the present work, because the solutions we construct do satisfy it.

Recently, Kusner, Grosse-Brauckmann and Sullivan have shown that modulo Euclidean motions, $\mathcal{M}_{0,3}$ is a 3 -ball [4]; it is plausible from their work that this particular moduli space contains no degenerate elements.

It is a consequence of the present work and our more recent paper [11] concerning connected sums of nondegenerate CMC surfaces that many more 
of these CMC moduli spaces contain nondegenerate elements and thus attain their correct dimension.

We now state our main result in more detail. This result is simply that CMC surfaces may be constructed out of certain building blocks in a specified and controlled manner. There are only two types of building blocks: Delaunay surfaces (or more precisely, halves of Delaunay surfaces) and minimal $k$-noids. The former we have already encountered; on the other hand, a minimal $k$-noid is by definition a complete minimal surface $\Sigma$ of finite total curvature with $k$ ends. Denote the moduli space of minimal $k$-noids of genus $g$ by $\mathcal{H}_{g, k}$. This space (or rather, a very closely associated one) has been studied by Perez and Ros [17], and they prove the result corresponding to that of [9] that this space is real analytic of virtual dimension $3 k$. Elements of these spaces have been shown to exist by the classical Weierstrass method, and more recently elements have been constructed for $g$ very large by Kapouleas by a desingularization scheme [6] and a connected sum result, analogous to that in [11], has been obtained by S. D. Yang [21]. Again there is a notion of nondegeneracy of such surfaces, and a surface $\Sigma$ is a smooth point in its corresponding moduli space precisely when it satisfies this nondegeneracy condition. Using the Weierstrass representation it is possible to establish the existence of nondegenerate minimal $k$-noids. For example, recently Cosin and Ros [1] have proved the existence of nondegenerate minimal $k$-noids of genus 0 with specified weight parameters (in the sense described later here) for every $k$. Later in this paper, in $\S 8.3$, we survey what is currently known about the existence of nondegenerate $k$-noids. Finally, as noted earlier, any end of an element $\Sigma \in \mathcal{H}_{g, k}$ is asymptotic to the end of a plane or a catenoid. Again, we decompose

$$
\mathcal{H}_{g, k}=\mathcal{H}_{g, k}^{s} \cup \mathcal{H}_{g, k}^{m} \cup \mathcal{H}_{g, k}^{0}
$$

where $\mathcal{H}_{g, k}^{s}$ is the set of surfaces all of whose ends are asymptotic to catenoids all with the same orientation, $\mathcal{H}_{g, k}^{m}$ is the set of surfaces whose ends are asymptotic to catenoids with different orientations and $\mathcal{H}_{g, k}^{0}$ is the set of surfaces having at least one planar end.

We may now state the main result of this paper.

Theorem 1. Fix any $\Sigma_{0} \in \mathcal{H}_{g, k}^{s} \cup \mathcal{H}_{g, k}^{m}$ and assume that $\Sigma_{0}$ is nondegenerate. Then there exist two distinct families of CMC surfaces $\Sigma_{\varepsilon}^{+}, \Sigma_{\varepsilon}^{-} \in \mathcal{M}_{g, k}$, $\varepsilon \in\left(0, \varepsilon_{0}\right)$, constructed by gluing half-Delaunay surfaces onto each end of the dilated surface $\varepsilon \Sigma_{0}$.

The surfaces $\Sigma_{\varepsilon}^{ \pm}$have the property that for any $R>0$, the dilated surfaces $\varepsilon^{-1} \Sigma_{\varepsilon}^{ \pm}$restricted to the ball $B_{R}(0)$ converge in the $\mathcal{C}^{\infty}$ topology to the 
restriction of $\Sigma_{0}$ to $B_{R}(0)$, as $\varepsilon$ tends to 0 . Furthermore, if $\Sigma_{0} \in \mathcal{H}_{g, k}^{s}$ then $\Sigma_{\varepsilon}^{+} \in \mathcal{M}_{g, k}^{u}$ and $\Sigma_{\varepsilon}^{-} \in \mathcal{M}_{g, k}^{n}$, while if $\Sigma_{0} \in \mathcal{H}_{g, k}^{m}$ then $\Sigma_{\varepsilon}^{ \pm} \in \mathcal{M}_{g, k}^{m}$. Finally, $\Sigma_{\varepsilon}^{ \pm}$are regular points of $\mathcal{M}_{g, k}$.

The two distinct families of CMC surfaces in $\mathcal{M}_{g, k}$ associated to each $\Sigma_{0}$ correspond to gluing half-Delaunay surfaces (which may be either unduloids or nodoids) onto each end of the dilated surface $\varepsilon \Sigma_{0}$ according to the chosen orientation for $\Sigma_{0}$. If all ends of $\Sigma_{0}$ are asymptotic to similarly oriented catenoids, then we attach either all unduloids or all nodoids, whereas if the ends of $\Sigma_{0}$ are asymptotic to differently oriented catenoids, and if we attach unduloids to the subset of ends which correspond to one orientation, then we must attach nodoids to the other ends, which correspond to the other orientation.

Our proof has some novel features. Rather than finding solutions as perturbations off of degenerating families of approximate solutions, as has been common in such constructions, we instead find (infinite dimensional) families of CMC surfaces as normal graphs over each of the component pieces, the half-Delaunay surfaces and a truncated $k$-noid. By studying the Cauchy data of the functions producing these normal graphs, and prove that we may match the Cauchy data from the inner piece (the graph over the $k$-noid) with that from the ends, thus one advantage of this procedure is that more of the technical complications caused by the nonlinearities are avoided than would be otherwise. Another important issue is that in previous constructions ([5], [7], [21]) one must face the delicate issue of balancing forces, or creating small nonvanishing forces in the approximate configurations. We do not need to deal with this issue here because we implicitly use the balancing of forces already existing in the minimal $k$-noids.

This paper achieves two main geometric goals. The first is that we establish a specific method for passing from minimal $k$-noids to complete CMC surfaces with $k$ ends. In fact, we get an embedding of (the nondegenerate elements in) $\left(\mathcal{H}_{g, k}^{s} \cup \mathcal{H}_{g, k}^{m}\right) \times(0, \epsilon)$ into $\mathcal{M}_{g, k}$; this parametrizes an end of this latter moduli space. Furthermore, combining the result here with the connected sum construction of [11], we also establish the existence of nondegenerate elements in $\mathcal{M}_{g, k}$ for many values of $g$ and $k$. We shall return to this latter problem, and a study of various other aspects of these moduli spaces, in a forthcoming paper.

The plan of this paper is as follows. We first discuss the Delaunay surfaces in some detail, collecting and proving various technical properties concerning them that we require later, specifically those concerned with their behavior in the singular limit, as they approach the bead of spheres. This 
is followed by the analysis of the Jacobi (i.e. linearized CMC) operator, especially in this singular limit, for the half-Delaunay surfaces. We then use this to discuss the full family of CMC surfaces in a neighborhood of these rotationally invariant surfaces, as usual, keeping careful track of the behavior in the limit. Next, we turn to a discussion of minimal surfaces with $k$ catenoidal ends, i.e. the $k$-noids, briefly reviewing their geometry and then treating the relevant aspects of the linear analysis of their Jacobi operator. After that, we can approach the family of CMC surfaces obtained as normal graphs over suitable truncations of these $k$-noids. At last, we can put all of this together and prove that it is possible to match the Cauchy data, and so obtain the proof of the main theorem.

Acknowledgment. This paper was written when the second author was visiting Stanford University. He would like to take this opportunity to thank the American Institute of Mathematics (AIM) and Stanford's Mathematics Department for their support and hospitality.

The authors would also like to thank K. Grosse-Brauckmann and R. Kusner for pointing out that our construction should be extended to cover immersed as well as embedded ends.

\section{Notation, conventions and definitions.}

We recall some basic facts about the geometry of immersed surfaces, and review various ways the equations for constant mean curvature may be specified. Some good references for this material are the book by Osserman [16] and the survey article by Wente [20].

Suppose that $\Sigma$ is given as the image of a regular immersion $\mathbf{x}: \mathcal{U} \longrightarrow \mathbb{R}^{3}$. Here $\mathcal{U}$ is an open set in $\mathbb{R}^{2}$ with coordinates $u=\left(u_{1}, u_{2}\right)$. The unit normal to $\Sigma$ is defined to be

$$
\nu\left(u_{1}, u_{2}\right)=\frac{\partial_{u_{1}} \mathbf{x} \times \partial_{u_{2}} \mathbf{x}}{\left\|\partial_{u_{1}} \mathbf{x} \times \partial_{u_{2}} \mathbf{x}\right\|}
$$

and the components of the first and second fundamental forms $g$ and $B$ are then

$$
\begin{aligned}
E & =\left\langle\partial_{u_{1}} \mathbf{x}, \partial_{u_{1}} \mathbf{x}\right\rangle, F=\left\langle\partial_{u_{1}} \mathbf{x}, \partial_{u_{2}} \mathbf{x}\right\rangle, G=\left\langle\partial_{u_{2}} \mathbf{x}, \partial_{u_{2}} \mathbf{x}\right\rangle \\
L & =\left\langle\partial_{u_{1} u_{1}}^{2} \mathbf{x}, \nu\right\rangle, M=\left\langle\partial_{u_{1} u_{2}}^{2} \mathbf{x}, \nu\right\rangle, N=\left\langle\partial_{u_{2} u_{2}}^{2} \mathbf{x}, \nu\right\rangle .
\end{aligned}
$$

The principal curvatures $k_{1}$ and $k_{2}$ are the eigenvalues of $B$ relative to $g$. The mean curvature is defined to be the sum (not the average) of the 
principal curvatures, $H:=k_{1}+k_{2}$, and the Gauss curvature is their product, $K:=k_{1} k_{2}$.

We shall almost always be using orthogonal parameterizations (that is to say, parameterizations for which $F=0$ ), in which case the formulæ for $H$ and $K$ reduce to:

$$
H=\frac{L+N}{E}, \quad K=\frac{L N-M^{2}}{E^{2}} .
$$

The important equations of surface theory are the Gauss and Codazzi equations, which link the intrinsic and extrinsic geometry of $\Sigma$. Rather than write these down in general, we consider the special case where the parameterization is isothermal, so that $E=G:=e^{2 \omega}$ and $F=0$. Let $u=u_{1}+i u_{2}$ be the corresponding complex coordinate, and define the Hopf differential

$$
\phi(u) d u^{2}=\left(\frac{1}{2}(L-N)-i M\right) d u^{2} .
$$

The principal curvatures are then given by

$$
k_{1}=\frac{H}{2}-|\phi| e^{-2 w}, \quad k_{2}=\frac{H}{2}+|\phi| e^{-2 w} .
$$

If $\Sigma$ is CMC, so $H$ is constant, then the Codazzi equations are equivalent to the holomorphy of this differential. The Gauss equation is simply

$$
\Delta \omega+\frac{H^{2}}{4} e^{2 \omega}-|\phi|^{2} e^{-2 \omega}=0 .
$$

\section{Delaunay surfaces.}

We now make a detailed study of the first of the basic building blocks we use later, the Delaunay surfaces of revolution. As already mentionned in the introduction, Delaunay surfaces can be classified into two different types : the embedded Delaunay surfaces which are called unduloids and the immersed Delaunay surfaces which are called nodoids.

\subsection{Definition and basic equations.}

The Delaunay surfaces mentioned in the introduction are surfaces of revolution, and so we use cylindrical coordinates. In particular, if the axis of rotation is the vertical one, and if $t$ is a linear coordinate along this axis and 
$\theta$ is the angular variable around it, then we consider surfaces $\Sigma$ given (at least locally) by the parametrization

$$
\mathbf{x}(t, \theta):=(\rho(t) \cos \theta, \rho(t) \sin \theta, t) .
$$

The condition that such a surface has constant mean curvature 1 (or -1 depending on the chosen orientation) gives an ordinary differential equation for the function $\rho$, and solutions of this equation correspond to the Delaunay surfaces.

To obtain this ODE, first note that the unit normal of $\Sigma$ at $\mathbf{x}(t, \theta)$ is

$$
\nu(t, \theta):=\frac{1}{\sqrt{1+\rho_{t}^{2}}}\left(-\cos \theta,-\sin \theta, \rho_{t}\right)
$$

where subscripts denote derivatives, and then that the metric tensor and second fundamental form are given by

$$
g=\left(1+\rho_{t}^{2}\right) d t^{2}+\rho^{2} d \theta^{2}, \quad B=-\frac{\rho_{t t}}{\sqrt{1+\rho_{t}^{2}}} d t^{2}+\frac{\rho}{\sqrt{1+\rho_{t}^{2}}} d \theta^{2}
$$

It follows that the mean curvature is given by the expression

$$
H=-\rho_{t t}\left(1+\rho_{t}^{2}\right)^{-3 / 2}+\rho^{-1}\left(1+\rho_{t}^{2}\right)^{-1 / 2},
$$

and so the condition $H \equiv 1$ leads to the equation

$$
\rho_{t t}-\frac{1}{\rho}\left(1+\rho_{t}^{2}\right)+\left(1+\rho_{t}^{2}\right)^{3 / 2}=0
$$

while the condition $H \equiv-1$ yields

$$
\rho_{t t}-\frac{1}{\rho}\left(1+\rho_{t}^{2}\right)-\left(1+\rho_{t}^{2}\right)^{3 / 2}=0 .
$$

There are two special solutions of (3.4) that can be determined immediately. The first is the constant solution $\rho_{1} \equiv 1$, the cylindrical graph of which is the cylinder of radius 1 . The other, $\rho_{0}=\sqrt{4-(t-2)^{2}}$, for $|t-2| \leq 2$, corresponds to the sphere of radius 2 centered at $(0,0,2)$. The singular limit of the Delaunay surfaces mentioned in the introduction corresponds to the periodic extension of $\rho_{0}$ to all of $\mathbb{R}$. 
3.1.1. Embedded Delaunay surfaces : the unduloids. For all $\varepsilon \in$ $(0,1)$, we define $\rho_{\varepsilon}$ to be the solution of (3.4) which attains its minimum value $\rho_{\varepsilon}(0)=\varepsilon$ at $t=0$. By differentiating, we see that if $\rho$ is a solution of (3.4) then

$$
\mathcal{H}\left(\rho, \rho_{t}\right):=\rho^{2}-\frac{2 \rho}{\sqrt{1+\rho_{t}^{2}}},
$$

is constant. In particular, $\mathcal{H}\left(\rho_{\varepsilon},\left(\rho_{\varepsilon}\right)_{t}\right)=\varepsilon(\varepsilon-2)<0$. Introduce the new parameter $\tau>0$ by $\tau^{2}:=\varepsilon(2-\varepsilon)$, so that $\varepsilon=1-\sqrt{1-\tau^{2}}$ and $\tau \in(0,1)$ as well. We then deduce immediately the

Proposition 1. For all $\varepsilon \in(0,1)$, the solution $\rho_{\varepsilon}$ of (3.4) with $\rho_{\varepsilon}(0)=\varepsilon$, $\left(\rho_{\varepsilon}\right)_{t}(0)=0$ is periodic and varies between the limits

$$
\varepsilon=1-\sqrt{1-\tau^{2}} \leq \rho_{\varepsilon} \leq 1+\sqrt{1-\tau^{2}}=2-\varepsilon .
$$

In particular, $\rho_{\varepsilon} \leq 2$ for all $\varepsilon$.

These solutions constitute the (embedded) Delaunay family; the surfaces determined by them, as well as their images under Euclidean motions, are Delaunay surfaces called unduloids.

To simplify notation, we often drop the subscript $\varepsilon$ (which should not be confused with the standard partial derivative notation). We also introduce a new parameterization, changing both the independent and dependent variables, which simplifies the study of the $\rho_{\varepsilon}$. A change of independent variable corresponds to the introduction of a function $t=k(s)$, which should be a diffeomorphism of $\mathbb{R}$ onto itself. The function $k$ is chosen so that the corresponding parameterization

$$
(s, \theta) \longrightarrow(\rho \circ k \cos \theta, \rho \circ k \sin \theta, k),
$$

is isothermal. This corresponds to the condition

$$
\left(1+\rho_{t}^{2}\right) k_{s}^{2}=\rho^{2} .
$$

With the initial condition $k(0)=0$ and noting that $k_{s}>0$, then $\rho$ uniquely determines $k$. Also, $k_{s} \neq 0$ (so long as $\rho \neq 0$, which is always the case here), and so using the periodicity of $\rho$ we see that $k$ must be a diffeomorphism. Now, use the parameter $\tau \in(0,1)$ from above and define the function $\sigma$ by

$$
\tau e^{\sigma}=\rho \circ k .
$$


A brief calculation shows that

$$
1+\rho_{t}^{2}=\frac{1}{1-\sigma_{s}^{2}}, \quad \text { and } \quad \rho_{t t}=\frac{\sigma_{s s}}{\tau e^{\sigma}\left(1-\sigma_{s}^{2}\right)^{2}} .
$$

In terms of the new variable $s$ and function $\sigma$, the first and second fundamental forms are now

$$
g=\tau^{2} e^{2 \sigma}\left(d s^{2}+d \theta^{2}\right), \quad B=-\frac{\tau e^{\sigma} \sigma_{s s}}{\sqrt{1-\sigma_{s}^{2}}} d s^{2}+\tau e^{\sigma} \sqrt{1-\sigma_{s}^{2}} d \theta^{2}
$$

and (3.4) becomes

$$
\sigma_{s s}+\tau e^{\sigma} \sqrt{1-\sigma_{s}^{2}}-\left(1-\sigma_{s}^{2}\right)=0 .
$$

We can now see that this parameterization is indeed simpler.

Proposition 2. The function $\sigma$ defined by (3.7) satisfies the equation

$$
\sigma_{s s}+\frac{\tau^{2}}{2} \sinh (2 \sigma)=0
$$

and in fact

$$
\sigma_{s}^{2}+\tau^{2} \cosh ^{2} \sigma=1
$$

Conversely, for any $\tau \in(0,1)$, suppose $\sigma$ satisfies (3.12) (and hence also (3.11)) with $\tau^{2} \cosh ^{2} \sigma(0)=1$, and that $t=k(s)$, where $k(0)=0$ and

$$
k_{s}=\frac{\tau^{2}}{2}\left(1+e^{2 \sigma}\right)
$$

then, the function $\rho$ defined by $\rho \circ k=\tau e^{\sigma}$ satisfies (3.4) and $\rho(0)=\varepsilon$ where $\varepsilon=1-\sqrt{1-\tau^{2}}$.

Proof. By definition of $\tau$, we have

$$
\mathcal{H}\left(\rho, \rho_{t}\right)=\rho^{2}-\frac{2 \rho}{\sqrt{1+\rho_{t}^{2}}}=-\tau^{2} .
$$

Since $\rho \circ k=\tau e^{\sigma}$ and, by (3.8), $1+\rho_{t}^{2}=\left(1-\sigma_{s}^{2}\right)^{-1}$, this equality becomes

$$
2 \tau e^{\sigma} \sqrt{1-\sigma_{s}^{2}}-\tau^{2} e^{2 \sigma}=\tau^{2},
$$

which yields directly (3.12), and hence (3.11), by differentiation. 
The converse, that starting from $\sigma$ and $\tau$, then defining $t=k(s)$ as in the statement of the Proposition, the corresponding function $\rho$ satisfies (3.4) is a straightforward calculation which we leave to the reader. Notice that the Gauss and Codazzi equations are automatically fulfilled.

Remark 1. Translating back to the notation of $\S 2$, the log of the conformal factor $\omega$ and the norm of the coefficient function of the Hopf differential are given by

$$
\omega=\sigma+\log \tau \quad \text { and } \quad|\phi|=\frac{\tau^{2}}{2}
$$

c.f. $(2.2)$ and (2.3).

Henceforth, the functions $\rho, \sigma$ and $k$ will always be related in the manner dictated by this Proposition; furthermore, the dependence on the parameters $\varepsilon$ and $\tau$ will not always be written explicitly, but we shall use them interchangeably. We shall call either of these parameters the necksize of the corresponding Delaunay solution.

3.1.2. Immersed Delaunay surfaces : the nodoids. For all $\varepsilon>0$, we now define $\bar{\rho}_{\varepsilon}$ to be the solution of (3.5) which attains its minimum value $\bar{\rho}_{\varepsilon}(0)=\varepsilon$ at $t=0$. By differentiating, we see that if $\bar{\rho}$ is a solution of (3.5) then

$$
\overline{\mathcal{H}}\left(\bar{\rho}, \bar{\rho}_{t}\right):=\bar{\rho}^{2}+\frac{2 \bar{\rho}}{\sqrt{1+\bar{\rho}_{t}^{2}}}
$$

is constant. In particular, $\overline{\mathcal{H}}\left(\bar{\rho}_{\varepsilon},\left(\bar{\rho}_{\varepsilon}\right)_{t}\right)=\varepsilon(\varepsilon+2)>0$. Introduce the new parameter $\tau>0$ by $\tau^{2}:=\varepsilon(2+\varepsilon)$, so that $\varepsilon=\sqrt{1+\tau^{2}}-1$ and $\tau>0$ as well. We then deduce immediately the

Proposition 3. The solution $\bar{\rho}_{\varepsilon}$ of (3.5) with $\bar{\rho}_{\varepsilon}(0)=\varepsilon,\left(\bar{\rho}_{\varepsilon}\right)_{t}(0)=0$ is defined in some interval $\left(-\bar{T}_{\varepsilon}, \bar{T}_{\varepsilon}\right)$, with $0<\bar{T}_{\varepsilon}<+\infty$, and varies between the limits

$$
\varepsilon=\sqrt{1+\tau^{2}}-1 \leq \bar{\rho}_{\varepsilon} \leq \tau=\sqrt{\varepsilon^{2}+2 \varepsilon} .
$$

Moreover

$$
\lim _{t \rightarrow \pm \bar{T}_{\varepsilon}} \bar{\rho}_{\varepsilon}=\tau, \quad \text { and } \quad \lim _{t \rightarrow \pm \bar{T}_{\varepsilon}}\left(\bar{\rho}_{\varepsilon}\right)_{t}= \pm \infty
$$


As for unduloids, we introduce an isothermal parameterization

$$
(s, \theta) \longrightarrow\left(\tau e^{\bar{\sigma}} \cos \theta, \tau e^{\bar{\sigma}} \sin \theta, \bar{k}\right),
$$

of these surfaces. Proposition 2 becomes

Proposition 4. The function $\bar{\sigma}$ is negative and satisfies the equation

$$
\bar{\sigma}_{s s}+\frac{\tau^{2}}{2} \sinh (2 \bar{\sigma})=0
$$

and in fact

$$
\bar{\sigma}_{s}^{2}+\tau^{2} \sinh ^{2} \bar{\sigma}=1 .
$$

Conversely, for any $\tau>0$, if $\bar{\sigma}$ is negative and satisfies (3.15) (and hence also (3.14)) with $\tau^{2} \sinh ^{2} \bar{\sigma}(0)=1$, and if $t=\bar{k}(s)$, where $\bar{k}(0)=0$ and

$$
\bar{k}_{s}=\frac{\tau^{2}}{2}\left(1-e^{2 \bar{\sigma}}\right),
$$

then the function $\bar{\rho}$ which is defined by $\bar{\rho} \circ \bar{k}=\tau e^{\bar{\sigma}}$ satisfies (3.5) and $\bar{\rho}(0)=\varepsilon$ where $\varepsilon=\sqrt{1+\tau^{2}}-1$.

Proof. We write $\bar{\rho} \circ \bar{k}=\tau e^{\bar{\sigma}}$. Notice that (3.8) is still available. The fact that $\overline{\mathcal{H}}\left(\bar{\rho}, \bar{\rho}_{t}\right)$ is constant equal to $\tau^{2}$ yields

$$
2 \tau e^{\bar{\sigma}} \sqrt{1-\bar{\sigma}_{s}^{2}}+\tau^{2} e^{2 \bar{\sigma}}=\tau^{2},
$$

which gives (3.15), and hence (3.14) by differentiation.

Finally, for all $\varepsilon>0$, we define $\tilde{\rho}_{\varepsilon}$ to be the solution of (3.4) which attains its maximum value $\tilde{\rho}_{\varepsilon}(0)=2+\varepsilon$ at $t=0$. Again, if $\tilde{\rho}$ is a solution of (3.4), we know that

$$
\mathcal{H}\left(\tilde{\rho}, \tilde{\rho}_{t}\right):=\tilde{\rho}^{2}-\frac{2 \tilde{\rho}}{\sqrt{1+\tilde{\rho}_{t}^{2}}}
$$

is constant. In particular, $\mathcal{H}\left(\tilde{\rho}_{\varepsilon},\left(\tilde{\rho}_{\varepsilon}\right)_{t}\right)=\varepsilon(\varepsilon+2)=\tau^{2}>0$. We then deduce immediately the 
Proposition 5. For all $\varepsilon>0$, the solution $\tilde{\rho}_{\varepsilon}$ of (3.4) with $\tilde{\rho}_{\varepsilon}(0)=2+\varepsilon$, $\left(\tilde{\rho}_{\varepsilon}\right)_{t}(0)=0$ is defined in some interval $\left(-\tilde{T}_{\varepsilon}, \tilde{T}_{\varepsilon}\right)$, with $0<\tilde{T}_{\varepsilon}<+\infty$, and varies between the limits

$$
\sqrt{\varepsilon(\varepsilon+2)}=\tau \leq \tilde{\rho}_{\varepsilon} \leq 1+\sqrt{1+\tau^{2}}=2+\varepsilon .
$$

Moreover

$$
\lim _{t \rightarrow \pm \tilde{T}_{\varepsilon}} \tilde{\rho}_{\varepsilon}=\tau, \quad \text { and } \quad \lim _{t \rightarrow \pm \tilde{T}_{\varepsilon}}\left(\tilde{\rho}_{\varepsilon}\right)_{t}=\mp \infty .
$$

Again, we introduce an isothermal parameterization

$$
(s, \theta) \longrightarrow\left(\tau e^{\tilde{\sigma}} \cos \theta, \tau e^{\tilde{\sigma}} \sin \theta, \tilde{k}\right),
$$

We have

Proposition 6. The function $\tilde{\sigma}$ is positive and satisfies the equation

$$
\tilde{\sigma}_{s s}+\frac{\tau^{2}}{2} \sinh (2 \tilde{\sigma})=0
$$

and in fact

$$
\tilde{\sigma}_{s}^{2}+\tau^{2} \sinh ^{2} \tilde{\sigma}=1
$$

Conversely, for any $\tau>0$, if $\tilde{\sigma}$ is positive and satisfies (3.18) (and hence also (3.17)) with $\tau^{2} \sinh ^{2} \tilde{\sigma}(0)=1$, and if $t=\tilde{k}(s)$, where $\tilde{k}(0)=0$ and

$$
\tilde{k}_{s}=\frac{\tau^{2}}{2}\left(1-e^{2 \tilde{\sigma}}\right)
$$

then, the function $\tilde{\rho}$ defined by $\tilde{\rho} \circ \tilde{k}=\tau e^{\tilde{\sigma}}$ satisfies (3.4) and $\tilde{\rho}(0)=2+\varepsilon$ where $\varepsilon=\sqrt{1+\tau^{2}}-1$.

Proof. We write $\tilde{\rho} \circ \tilde{k}=\tau e^{\tilde{\sigma}}$. The fact that $\mathcal{H}\left(\tilde{\rho}, \tilde{\rho}_{t}\right)$ is constant equal to $\tau^{2}$ yields

$$
2 \tau e^{\tilde{\sigma}} \sqrt{1-\tilde{\sigma}_{s}^{2}}-\tau^{2} e^{2 \tilde{\sigma}}=-\tau^{2},
$$

and again this gives (3.15), and hence (3.14).

The two solutions $\bar{\rho}_{\varepsilon}$ and $\tilde{\rho}_{\varepsilon}$ can be glued together by translating the graph of $\tilde{\rho}_{\varepsilon}$ along the $z$ axis by the amount $\bar{T}_{\varepsilon}-\tilde{T}_{\varepsilon}$ and then this surface can 
be extended by periodicity. Notice that the fact that one surface has mean curvature 1 and the other -1 is consistent with the different orientations chosen for the gluing. These solutions constitute the (immersed) Delaunay family; the surfaces determined by them, as well as their images under Euclidean motions, are Delaunay surfaces which are called nodoids.

Notice that for the corresponding isothermal parameterizations of the two pieces of the nodoid, the two functions $\bar{\sigma}$ and $\tilde{\sigma}$ are solutions of the same equation. Therefore, we shall refer to both of them as $\bar{\sigma}$ and $\bar{k}$, so that the parameterization is now global, keeping in mind that positive $\bar{\sigma}$ corresponds to $\tilde{\rho}$.

\subsection{Uniform estimates for Delaunay solutions in the singular limit.}

In this section we present a series of technical lemmata regarding the behavior of various quantities associated to the Delaunay solutions as $\varepsilon$ (or $\tau$ ) tends to zero. Some of the estimates below are easier to obtain for $\rho$ or $\bar{\rho}$ and some for $\sigma$ or $\bar{\sigma}$, and we shall use these functions interchangeably. We first estimate the period of $\sigma$; the corresponding estimate for $\rho$ is not required later so we merely state it and refer to [5] for its proof. Then we obtain some simple 'global' estimates for $\rho$, which are rather weak, but frequently useful, as well as a corresponding simple estimate for $\sigma$. Finer estimates for $\rho$ when $t$ is not too large then lead to a good comparison between the variables $s$ and $t$.

3.2.1. Unduloids in the singular limit. In this section, we shall only consider unduloids and we shall do so without further comment. We start with the simple

Proposition 7. Let $S_{\varepsilon}$ and $T_{\varepsilon}$ denote the periods of $\sigma$ and $\rho$, respectively. Then as functions of $\tau$ and $\varepsilon$,

$$
S_{\varepsilon}=-4 \log \tau+O(1)=-2 \log \varepsilon+O(1)
$$

and

$$
T_{\varepsilon}=4+\tau^{2} \log (1 / \tau)+O\left(\tau^{2}\right)=4+2 \varepsilon \log (1 / \varepsilon)+O(\varepsilon)
$$


Proof. As stated above, we only check the statement about $S_{\varepsilon}$. First, using (3.12), we see that

$$
\frac{1}{4} S_{\varepsilon}=\int_{\sigma(0)}^{0} \frac{1}{\sqrt{1-\tau^{2} \cosh ^{2} x}} d x .
$$

Expand the denominator into exponentials and change variables, setting $u=e^{x}$. Then, letting

$$
A(\tau):=e^{\sigma(0)}=\frac{1}{\tau}-\sqrt{\frac{1}{\tau^{2}}-1}=\frac{\tau}{2}+O\left(\tau^{3}\right),
$$

this becomes

$$
\frac{1}{4} S_{\varepsilon}=\int_{A(\tau)}^{1} \frac{1}{\sqrt{u^{2}-\frac{1}{4} \tau^{2}\left(u^{2}+1\right)^{2}}} d u .
$$

Changing variables once again, reduces this to an integral of the form

$$
\int_{\tau / 2}^{1-O\left(\tau^{2}\right)} \frac{1}{\sqrt{v^{2}-\tau^{2} / 4}} d v
$$

Finally, this last integral may be computed explicitly, and equals $-\log \tau+$ $O(1)$. The estimate for $S_{\varepsilon}$ in terms of $\varepsilon$ follows from the relationship between $\varepsilon$ and $\tau$.

To place the next result into context, note that one limiting solution of the basic equation (3.4) is $\rho_{0}:=\sqrt{4-(t-2)^{2}}$, the equation for the sphere. Also, the catenoid of necksize $\varepsilon$ (which is a solution of the equation corresponding to (3.3) when $H=0$ ) is given as a cylindrical graph by the function $\rho_{c}:=\varepsilon \cosh (t / \varepsilon)$. The function $\rho$ may be compared to each of these solutions.

Proposition 8. For any $\varepsilon \in(0,1)$, the Delaunay solution $\rho:=\rho_{\varepsilon}$ satisfies the following bounds

$$
\varepsilon \leq \rho \leq \varepsilon \cosh (t / \varepsilon)
$$

(3.21) $1+\rho_{t}^{2} \leq \frac{\rho^{2}}{\varepsilon^{2}} \quad$ (comparison with the equation of a catenoid),

$$
\rho^{2}\left(1+\rho_{t}^{2}\right) \leq 4 \quad \text { (comparison with the equation of a sphere), }
$$

for any $t \in \mathbb{R}$. 
Proof. It is clear from (3.12) that $\sigma$ is monotone increasing on $\left[0, S_{\varepsilon} / 2\right]$ and monotone decreasing on $\left[S_{\varepsilon} / 2, S_{\varepsilon}\right]$. Correspondingly, $\rho$ is monotone increasing on $\left[0, T_{\varepsilon} / 2\right]$ and monotone decreasing on $\left[T_{\varepsilon} / 2, T_{\varepsilon}\right]$. In particular, its value at 0 is its absolute minimum, so the lower bound in (3.20) is valid.

Now multiply (3.4) by $2 \rho_{t}\left(1+\rho_{t}^{2}\right)^{-1}$ and integrate to get

$$
\log \left(1+\rho_{t}^{2}\right)=2 \log (\rho / \varepsilon)-2 \int_{0}^{t} \rho_{t} \sqrt{1+\rho_{t}^{2}} d u .
$$

Since $\rho_{t} \geq 0$ for $t \in\left[0, T_{\varepsilon} / 2\right]$, we get (3.21). Next, because $\rho \geq \varepsilon$, we may write $\rho=\varepsilon \cosh (w / \varepsilon)$. Inserting this into (3.21) leads to the inequality $w_{t}^{2} \leq 1$. Since $w(0)=0$, we conclude that $w \leq t$ for all $t \in\left[0, T_{\varepsilon} / 2\right]$ and the second part of (3.20) follows by periodicity.

Since the final estimate (3.22) is not required later, we shall not prove it here.

Now we come to the more refined estimates for $\rho$.

Proposition 9. There exists $c>0$ such that, for any $\varepsilon \in(0,1)$ and $|t| \leq$ $\frac{\varepsilon}{2} \log \left(\frac{c}{\varepsilon}\right)$, the Delaunay solution $\rho=\rho_{\varepsilon}$ satisfies

$$
|\rho-\varepsilon \cosh (t / \varepsilon)| \leq c \varepsilon^{2} e^{3|t| / \varepsilon} \quad \text { and } \quad\left|\rho_{t}-\sinh (t / \varepsilon)\right| \leq c \varepsilon e^{3|t| / \varepsilon} .
$$

Proof. Using the fact that $\tau^{2}=2 \varepsilon-\varepsilon^{2}$, we can rewrite the identity

$$
\rho^{2}-\frac{2 \rho}{\sqrt{1+\rho_{t}^{2}}}=-\tau^{2}
$$

as

$$
1+\rho_{t}^{2}=\left(\frac{\tau^{2}+\varepsilon^{2}}{\tau^{2}+\rho^{2}}\right)^{2} \frac{\rho^{2}}{\varepsilon^{2}} .
$$

Since $\rho \geq \varepsilon$ we already obtain

$$
0 \geq 1+\rho_{t}^{2}-\frac{\rho^{2}}{\varepsilon^{2}}=\frac{2 \tau^{2}+\varepsilon^{2}+\rho^{2}}{\left(\tau^{2}+\rho^{2}\right)^{2}} \frac{\rho^{2}}{\varepsilon^{2}}\left(\varepsilon^{2}-\rho^{2}\right) .
$$

Using (3.20), we can estimate

$$
\frac{2 \tau^{2}+\varepsilon^{2}+\rho^{2}}{\left(\tau^{2}+\rho^{2}\right)^{2}} \frac{\rho^{2}}{\varepsilon^{2}} \leq \frac{2}{\tau^{2}} \cosh ^{2}(t / \varepsilon) \leq \frac{2}{\varepsilon} \cosh ^{2}(t / \varepsilon) .
$$


Hence

$$
0 \geq 1+\rho_{t}^{2}-\frac{\rho^{2}}{\varepsilon^{2}} \geq \frac{2}{\varepsilon}\left(\varepsilon^{2}-\rho^{2}\right) \cosh ^{2}(t / \varepsilon)
$$

Set $\rho=\varepsilon \cosh (w / \varepsilon)$. The previous inequalities yield

$$
0 \geq w_{t}^{2}-1 \geq-2 \varepsilon \cosh ^{2}(t / \varepsilon) .
$$

Hence, we have finally proved that, for all $t \in[0,-\varepsilon \log (2 \varepsilon) / 2]$

$$
\left(1-2 \varepsilon \cosh ^{2}(t / \varepsilon)\right)^{1 / 2} \leq w_{t} \leq 1 .
$$

Now integrate this inequality, using that $(1-x)^{1 / 2} \geq 1-x$ when $0<x<1$ and $\cosh ^{2} x \leq e^{2|x|}$ for all $x \in \mathbb{R}$, to get

$$
-\varepsilon^{2} e^{2 t / \varepsilon} \leq-2 \varepsilon^{2} \int_{0}^{t / \varepsilon} \cosh ^{2} s d s \leq w-t \leq 0 .
$$

We conclude that for $t \in[0,-\varepsilon / 2 \log (2 \varepsilon)]$, we have

$$
\varepsilon \cosh \left(t / \varepsilon-\varepsilon e^{2 t / \varepsilon}\right) \leq \rho \leq \varepsilon \cosh (t / \varepsilon) .
$$

The estimate for $\rho$ follows at once. To get the estimate for $\rho_{t}$, use (3.24) and the relationship $\rho_{t}=w_{t} \sinh (w / \varepsilon)$.

We can finally give a quantitative estimate for the relationship between the variables $t$ and $s$, or equivalently, for the function $k$.

Proposition 10. For $|s|<S_{\varepsilon} / 8$, the function $t=k(s)$ admits the expansion

$$
k=\varepsilon s+\frac{\varepsilon^{2}}{8} e^{2 s}+O\left(\varepsilon^{2} \log \varepsilon\right)
$$

uniformly as $\varepsilon \rightarrow 0$.

Proof. It suffices to consider the case $t=k(s) \geq 0$. The estimate for $\rho$ from the last Proposition implies

$$
(\rho \circ k)^{2}=\frac{1}{4} \varepsilon^{2} e^{2 k / \varepsilon}+O\left(\varepsilon^{2}\right)+O\left(\varepsilon^{3} e^{4 k / \varepsilon}\right)+O\left(\varepsilon^{4} e^{6 k / \varepsilon}\right) .
$$


The errors here are all of size no greater than $O\left(\varepsilon^{2}\right)$ precisely when $|k| \leq$ $-\varepsilon / 4 \log \varepsilon$ (up to an additive constant). Assuming that $s_{0}>0$ is chosen so that this bound is satisfied, then by the definition of $\tau$ and $\sigma$,

$$
\tau^{2} e^{2 \sigma}=\frac{\varepsilon^{2}}{4} e^{2 k / \varepsilon}+O\left(\varepsilon^{2}\right)
$$

Now recall the definition of $k$ via its derivative from Proposition 2,

$$
k_{s}=\frac{\tau^{2}}{2}+\frac{\tau^{2}}{2} e^{2 \sigma}=\varepsilon+\frac{\varepsilon^{2}}{8} e^{2 k / \varepsilon}+O\left(\varepsilon^{2}\right) .
$$

Since $k \leq(\varepsilon / 4) \log (1 / \varepsilon)$, we obtain $e^{2 k / \varepsilon} \leq \varepsilon^{-1 / 2}$, and so $k_{s}=\varepsilon+O\left(\varepsilon^{3 / 2}\right)$ in this range. Integrate to get $k=\varepsilon s+O\left(\varepsilon^{3 / 2} s\right)$ for $0 \leq s \leq s_{0}$. From this equation, we see that $k \leq(\varepsilon / 4) \log (1 / \varepsilon)$ provided $s \leq S_{\varepsilon} / 8$, so that we may take $s_{0}$ to be this last value. Now use this formula for $k$ in terms of $s$ in the estimate for $k_{s}$ above to get that

$$
k_{s}=\varepsilon+\frac{\varepsilon^{2}}{8} e^{2 s}+O\left(\varepsilon^{2}\right)
$$

for $0 \leq s \leq S_{\varepsilon} / 8$. Integrating this, at last, gives the estimate of the Proposition.

Collecting the results of Proposition 9 and the result of Proposition 10, we obtain :

Proposition 11. There exists a constant $c>0$ independent of $\varepsilon$ such that the following inequalities hold

$$
\tau e^{\sigma} \geq c \varepsilon^{3 / 4}, \quad \tau^{2} \cosh (2 \sigma) \leq c \varepsilon^{1 / 2}, \quad \text { if } \quad s \in\left[S_{\varepsilon} / 8,3 S_{\varepsilon} / 8\right],
$$

and

$$
\tau^{2} \cosh (2 \sigma) \leq c \varepsilon^{2} e^{2 s}, \quad \text { if } \quad s \in\left[3 S_{\varepsilon} / 8, S_{\varepsilon} / 2\right] .
$$

Proof. Recall that $S_{\varepsilon}=-2 \log \varepsilon+O(1)$. It follows from Proposition 10 that

$$
k=\varepsilon s+O\left(\varepsilon^{3 / 2}\right) \quad \text { if } \quad s \in\left[0, S_{\varepsilon} / 8\right] .
$$

Therefore, using Proposition 9, we obtain the expansion

$$
\tau e^{\sigma}=\varepsilon \cosh s+O\left(\varepsilon^{3 / 2} e^{s}\right) \quad \text { and } \quad \tau e^{-\sigma}=\frac{2}{\cosh s}+O\left(\varepsilon^{1 / 2} e^{-s}\right),
$$


if $s \in\left[0, S_{\varepsilon} / 8\right]$. Since $\sigma$ is increasing in $\left[0, S_{\varepsilon} / 2\right]$, we conclude that

$$
\tau e^{\sigma} \geq \tau e^{\sigma\left(S_{\varepsilon} / 8\right)} \geq c \varepsilon^{3 / 4}
$$

for all $s \in\left[S_{\varepsilon} / 8, S_{\varepsilon} / 2\right]$. Similarly, since $|\sigma|$ is decreasing in $\left[0, S_{\varepsilon} / 4\right]$ and increasing in $\left[S_{\varepsilon} / 4, S_{\varepsilon} / 2\right]$, we get

$$
\tau^{2} \cosh (2 \sigma) \leq \tau^{2} \cosh \left(2 \sigma\left(S_{\varepsilon} / 8\right)\right) \leq c \varepsilon^{1 / 2},
$$

for all $s \in\left[S_{\varepsilon} / 8,3 S_{\varepsilon} / 8\right]$. Finally, since we always have

$$
\sigma(s)=-\sigma\left(S_{\varepsilon} / 2-s\right),
$$

it follows at once that, for all $s \in\left[S_{\varepsilon} / 2-S_{\varepsilon} / 8, S_{\varepsilon} / 2\right]$, we have

$$
\tau^{2} \cosh (2 \sigma(s))=\tau^{2} \cosh \left(2 \sigma\left(S_{\varepsilon} / 2-s\right)\right) \leq c e^{2 s-S_{\varepsilon}} \leq c \varepsilon^{2} e^{2 s} .
$$

This ends the proof of the Proposition.

We finally come to some simple estimates for $\sigma$. The first is that

$$
\tau^{2} \cosh (2 \sigma) \leq 2-\tau^{2}
$$

This follows trivially from multiplying $\cosh (2 \sigma)=2 \cosh ^{2} \sigma-1$ by $\tau^{2}$ and applying (3.12).

Next, define $\xi:=\tau \cosh \sigma$. This function is periodic of period $S_{\varepsilon} / 2$, attains its maximum value $\sup \xi=1$ at $s=0$, and its minimum inf $\xi=\tau$ at $s=S_{\varepsilon} / 4$. In addition, it is a solution of the equation

$$
\xi_{s s}=\left(1+\tau^{2}\right) \xi-2 \xi^{3}
$$

which satisfies

$$
\xi_{s}^{2}=\left(\xi^{2}-\tau^{2}\right)\left(1-\xi^{2}\right)
$$

Proposition 12. Suppose that $s_{\ell}$ is any sequence of real numbers, and that $\tau_{\ell} \rightarrow 0$. Let $\sigma_{\ell}$ denote the function $\sigma$ when $\tau=\tau_{\ell}$, we define $\xi_{\ell}(s)=$ $\tau_{\ell} \cosh \sigma_{\ell}\left(s+s_{\ell}\right)$ and $\tilde{\xi}_{\ell}(s)=\tau_{\ell}^{2} \cosh \left(2 \sigma_{\ell}\left(s+s_{\ell}\right)\right)$. Then there exists an $s_{0} \in \mathbb{R}$ and subsequences of the $\xi_{\ell}$ and $\tilde{\xi}_{\ell}$ which either converge uniformly to 0 or else converge respectively to $1 / \cosh \left(s+s_{0}\right)$ and $2 / \cosh ^{2}\left(s+s_{0}\right)$, uniformly on compact sets in $\mathbb{R}$. 
Proof. Since $\xi_{s}^{2}=\left(\xi^{2}-\tau^{2}\right)\left(1-\xi^{2}\right)$, and $|\xi| \leq 1$, we see that $\xi$ is bounded in $\mathcal{C}^{1}(\mathbb{R})$. Using $(3.27)$ we see that $\xi_{\ell}$ is bounded in $\mathcal{C}^{2}(\mathbb{R})$. This allows us to extract a subsequence which converges uniformly on compact subsets of $\mathbb{R}$ to a solution of

$$
\xi_{s s}=\xi-2 \xi^{3}
$$

which satisfies

$$
\xi_{s}^{2}=\xi^{2}\left(1-\xi^{2}\right) .
$$

For $\xi_{\ell}$, the claim follows since the only solutions of these equations are $\xi \equiv 0$ or $\xi=1 / \cosh \left(s+s_{0}\right)$ for some $s_{0} \in \mathbb{R}$. Finally, for $\tilde{\xi}_{\ell}$, it is sufficient to notice that

$$
\tilde{\xi}_{\ell}=2 \xi_{\ell}^{2}-\tau_{\ell}^{2}
$$

and the claim follows.

Because $\xi$ attains its supremum at $s=0$ we next conclude that

Corollary 1. As $\varepsilon \rightarrow 0$ the families of functions $\tau \cosh \sigma$ and $\tau^{2} \cosh (2 \sigma)$ converge to $1 / \cosh s$ and $2 / \cosh ^{2} s$, respectively, uniformly on compact sets.

In fact, we may improve the range on which the convergence in this last Corollary takes place.

Corollary 2. As $\varepsilon \rightarrow 0$,

$$
\tau \cosh \sigma=1 / \cosh s+O\left(\varepsilon^{1 / 2}\right), \quad \tau^{2} \cosh (2 \sigma)=2 / \cosh ^{2} s+O\left(\varepsilon^{1 / 2}\right),
$$

uniformly for $|s| \leq S_{\varepsilon} / 8$.

Proof. Note that $\xi=k_{s} / \rho \circ k$. The estimates here follow from inserting the estimates for $k$ and $\rho$ from Propositions 9 and 10 above.

Finally, since $\xi$ is decreasing on $\left[0, S_{\varepsilon} / 4\right]$ and increasing on $\left[S_{\varepsilon} / 4, S_{\varepsilon} / 2\right]$, we obtain using the previous Corollary, the

Proposition 13. For all $\eta>0$, there exists an $\varepsilon_{0} \in(0,1)$ and an $s_{0}>0$ such that whenever $\varepsilon \in\left(0, \varepsilon_{0}\right)$ and $N S_{\varepsilon} / 2+s_{0} \leq s \leq(N+1) S_{\varepsilon} / 2-s_{0}$ for some $N \in \mathbb{Z}$, then

$$
\xi=\tau \cosh \sigma \leq \eta \quad \text { and } \quad \tilde{\xi}=\tau^{2} \cosh (2 \sigma) \leq \eta .
$$


3.2.2. Nodoids in the singular limit. In this section, we derive for nodoids all the results which have been obtained for unduloids. Therefore, we shall only consider nodoids and we shall do so without further comment, focusing our attention on the results whose proofs need modifications. We start with the simple

Proposition 14. Let $\bar{S}_{\varepsilon}$ denote the periods of $\bar{\sigma}=\bar{\sigma}_{\varepsilon}$. Then, as functions of $\tau$ and $\varepsilon$,

$$
\bar{S}_{\varepsilon}=-4 \log \tau+O(1)=-2 \log \varepsilon+O(1) .
$$

Proof. The proof is identical to the proof of Proposition 7 once we have noticed that

$$
\frac{1}{4} \bar{S}_{\varepsilon}=\int_{\bar{\sigma}(0)}^{0} \frac{1}{\sqrt{1-\tau^{2} \sinh ^{2} x}} d x .
$$

We omit the details.

Now we come to the more refined estimates for $\bar{\rho}$.

Proposition 15. There exists $c>0$ such that, for any $\varepsilon>0$ and $|t| \leq$ $\frac{\varepsilon}{2} \log \left(\frac{c}{\varepsilon}\right)$, the Delaunay solution $\bar{\rho}=\bar{\rho}_{\varepsilon}$ satisfies

$$
|\bar{\rho}-\varepsilon \cosh (t / \varepsilon)| \leq c \varepsilon^{2} e^{3|t| / \varepsilon} \quad \text { and } \quad\left|\bar{\rho}_{t}-\sinh (t / \varepsilon)\right| \leq c \varepsilon e^{3|t| / \varepsilon} .
$$

Proof. Using the fact that $\tau^{2}=2 \varepsilon+\varepsilon^{2}$, we can rewrite the identity

$$
\bar{\rho}^{2}+\frac{2 \bar{\rho}}{\sqrt{1+\bar{\rho}_{t}^{2}}}=\tau^{2}
$$

as

$$
1+\bar{\rho}_{t}^{2}=\left(\frac{\tau^{2}-\varepsilon^{2}}{\tau^{2}-\bar{\rho}^{2}}\right)^{2} \frac{\bar{\rho}^{2}}{\varepsilon^{2}} .
$$

Since $\bar{\rho} \geq \varepsilon$ we already obtain

$$
0 \leq 1+\bar{\rho}_{t}^{2}-\frac{\bar{\rho}^{2}}{\varepsilon^{2}}=\frac{2 \tau^{2}-\varepsilon^{2}-\bar{\rho}^{2}}{\left(\tau^{2}-\bar{\rho}^{2}\right)^{2}} \frac{\bar{\rho}^{2}}{\varepsilon^{2}}\left(\bar{\rho}^{2}-\varepsilon^{2}\right) \leq \frac{2 \tau^{2}}{\left(\tau^{2}-\bar{\rho}^{2}\right)^{2}} \frac{\bar{\rho}^{2}}{\varepsilon^{2}}\left(\bar{\rho}^{2}-\varepsilon^{2}\right) .
$$


Let us choose $T_{m} \in\left(0, \bar{T}_{\varepsilon}\right)$ to be the supremum of all $T>0$ such that, for all $t \in[0, T]$

$$
\bar{\rho} \leq 2 \varepsilon \cosh (t / \varepsilon) \quad \text { and } \quad \bar{\rho} \leq \tau / \sqrt{2} .
$$

For all $t \in\left[0, T_{m}\right]$ we can thus estimate

$$
0 \leq 1+\bar{\rho}_{t}^{2}-\frac{\bar{\rho}^{2}}{\varepsilon^{2}} \leq \frac{8}{\tau^{2}}\left(\bar{\rho}^{2}-\varepsilon^{2}\right) \cosh ^{2}(t / \varepsilon) .
$$

Set $\bar{\rho}=\varepsilon \cosh (w / \varepsilon)$ in the previous inequalities, we obtain

$$
0 \leq w_{t}^{2}-1 \leq 8 \frac{\varepsilon^{2}}{2 \varepsilon+\varepsilon^{2}} \cosh ^{2}(t / \varepsilon) \leq 4 \varepsilon \cosh ^{2}(t / \varepsilon) .
$$

We have finally proved that, for all $t \in\left[0, T_{m}\right]$

$$
1 \leq w_{t} \leq\left(1+4 \varepsilon \cosh ^{2}(t / \varepsilon)\right)^{1 / 2},
$$

Now integrate this inequality, using that $(1+x)^{1 / 2} \leq 1+x / 2$ when $0<x$ and $\cosh ^{2} x \leq e^{2|x|}$ for all $x \in \mathbb{R}$, to get

$$
0 \leq w_{\varepsilon}-t \leq 2 \varepsilon^{2} \int_{0}^{t / \varepsilon} \cosh ^{2} s d s \leq \varepsilon^{2} e^{2 t / \varepsilon} .
$$

We conclude that for $0<t<T_{m}$, we have

$$
\varepsilon \cosh (t / \varepsilon) \leq \bar{\rho} \leq \varepsilon \cosh \left(t / \varepsilon+\varepsilon e^{2 t / \varepsilon}\right) .
$$

In particular we obtain that $T_{m} \geq \frac{\varepsilon}{2} \log (c / \varepsilon)$ for some constant $c>0$ independent of $\varepsilon>0$ and the estimate for $\bar{\rho}$ follows at once.

Here also we can give a quantitative estimate for the relationship between the variables $t$ and $s$, or equivalently, for the function $\bar{k}$.

Proposition 16. For $|s|<\bar{S}_{\varepsilon} / 8$, the function $t=\bar{k}(s)$ admits the expansion

$$
\bar{k}=\varepsilon s-\frac{\varepsilon^{2}}{8} e^{2 s}+O\left(\varepsilon^{2} \log \varepsilon\right)
$$

uniformly as $\varepsilon \rightarrow 0$. 
Proof. The proof is identical to the proof of Proposition 10, the only difference being that we must replace

$$
k_{s}=\frac{\tau^{2}}{2}+\frac{\tau^{2}}{2} e^{2 \sigma} \quad \text { by } \quad \bar{k}_{s}=\frac{\tau^{2}}{2}-\frac{\tau^{2}}{2} e^{2 \bar{\sigma}} .
$$

We leave the details to the reader.

Proposition 11 is left unchanged and reads

Proposition 17. There exists a constant $c>0$ independent of $\varepsilon$ such that the following inequalities hold

$$
\tau e^{\bar{\sigma}} \geq c \varepsilon^{3 / 4}, \quad \tau^{2} \cosh (2 \bar{\sigma}) \leq c \varepsilon^{1 / 2}, \quad \text { if } \quad s \in\left[\bar{S}_{\varepsilon} / 8,3 \bar{S}_{\varepsilon} / 8\right],
$$

and

$$
\tau^{2} \cosh (2 \bar{\sigma}) \leq c \varepsilon^{2} e^{2 s}, \quad \text { if } \quad s \in\left[3 \bar{S}_{\varepsilon} / 8, \bar{S}_{\varepsilon} / 2\right] .
$$

We finally come to some simple estimates for $\bar{\sigma}$. The first is that

$$
\tau^{2} \cosh (2 \bar{\sigma}) \leq 2+\tau^{2},
$$

This follows trivially from multiplying $\cosh (2 \bar{\sigma})=1+\cosh ^{2} \bar{\sigma}$ by $\tau^{2}$ and applying (3.15).

Next, define $\xi:=\tau \cosh \bar{\sigma}$. This function is periodic of period $\bar{S}_{\varepsilon} / 2$, attains its maximum value $\sup \xi=\sqrt{1+\tau^{2}}$ at $s=0$, and its minimum $\inf \xi=\tau$ at $s=\bar{S}_{\varepsilon} / 4$. In addition, it is a solution of the equation

$$
\xi_{s s}=\left(1+2 \tau^{2}\right) \xi-2 \xi^{3},
$$

which satisfies

$$
\xi_{s}^{2}=\left(\xi^{2}-\tau^{2}\right)\left(1+\tau^{2}-\xi^{2}\right) .
$$

The results of Proposition 12 and 13 as well as the results of Corollary 1 and 2 still hold for nodoids.

\section{The Jacobi operator on degenerating unduloids.}

In this section we first give an explicit expression for the linearization of the mean curvature operator about any one of the unduloids, and then proceed to develop its Fredholm theory on weighted Hölder spaces. This theory was already developed for weighted Sobolev spaces in [9], and the results are essentially identical. In particular, we need to find spaces on which this Jacobi operator is surjective. As usual, we also need this surjectivity with as good control as possible as the necksize shrinks. The results and proofs here are very close to those in [10]. 


\subsection{The Jacobi operator.}

Recall from the last section the cylindrical parameterization $\mathbf{x}_{\varepsilon}$ for the Delaunay surface $\Sigma_{\varepsilon}$ of necksize $\varepsilon$, and the corresponding expression for its unit normal $\nu_{\varepsilon}$. Given any function $w$ on $\Sigma_{\varepsilon}$, its normal graph

$$
\begin{aligned}
\mathbf{x}_{w} & =\mathbf{x}_{\varepsilon}+w \nu \\
& =\left(\left(\rho-\frac{w}{\sqrt{1+\rho_{t}^{2}}}\right) \cos \theta,\left(\rho-\frac{w}{\sqrt{1+\rho_{t}^{2}}}\right) \sin \theta, t+\frac{w}{\sqrt{1+\rho_{t}^{2}}}\right),
\end{aligned}
$$

gives a regular parametrization of a surface $\Sigma_{w}$, provided $w$ is sufficiently small. In terms of the coefficients of the first and second fundamental forms of this surface, the nonlinear operator we are interested in takes the form

$$
\mathcal{N}(w)=\frac{1}{2}-\frac{L_{w} G_{w}-2 M_{w} F_{w}+N_{w} E_{w}}{2\left(E_{w} G_{w}-F_{w}^{2}\right)} .
$$

It is well known that the linearization $\mathcal{L}_{\varepsilon}$ of $\mathcal{N}$ at $w=0$, which is usually called the Jacobi operator for $\Sigma_{\varepsilon}$, is given by

$$
\mathcal{L}_{\varepsilon}=\Delta_{\Sigma_{\varepsilon}}+\left|A_{\Sigma_{\varepsilon}}\right|^{2} .
$$

In terms of the parameterization above, this may be written as

$$
\mathcal{L}_{\varepsilon}=\frac{1}{\rho \sqrt{1+\rho_{t}^{2}}} \partial_{t}\left(\frac{\rho}{\sqrt{1+\rho_{t}^{2}}} \partial_{t}\right)+\frac{1}{\rho^{2}} \partial_{\theta \theta}^{2}+\frac{\rho^{2} \rho_{t t}^{2}+\left(1+\rho_{t}^{2}\right)^{2}}{\rho^{2}\left(1+\rho_{t}^{2}\right)^{3}} .
$$

This looks complicated, but fortunately, becomes simpler in the $(s, \theta)$ coordinate system introduced above. Now

$$
\mathcal{L}_{\varepsilon}=\frac{1}{\tau^{2} e^{2 \sigma}}\left(\partial_{s s}^{2}+\partial_{\theta \theta}^{2}+\tau^{2} \cosh (2 \sigma)\right) .
$$

Removing the factor $\left(\tau^{2} e^{2 \sigma}\right)^{-1}$, it will be sufficient to study the operator

$$
L_{\varepsilon} w=\partial_{s s}^{2}+\partial_{\theta \theta}^{2}+\tau^{2} \cosh (2 \sigma) .
$$

Our main goal now is to study the boundary problem

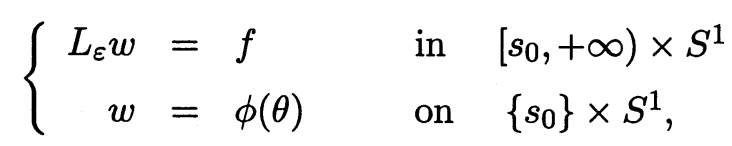


uniformly down to $\varepsilon=0$. Because of the rotational invariance of the operator $L_{\varepsilon}$, we may introduce the eigenfunction decomposition with respect to the cross-sectional Laplacian $\partial_{\theta \theta}^{2}$. In this way, we obtain operators

$$
L_{\varepsilon, j}=\partial_{s s}^{2}+\left(\tau^{2} \cosh 2 \sigma-j^{2}\right), \quad j \in \mathbb{Z} .
$$

Since we wish to deal only with real-valued functions, we shall use the eigenfunctions $\chi_{j}(\theta)=(1 / \sqrt{\pi}) \cos (j \theta)$ for $j>0, \chi_{j}(\theta)=(1 / \sqrt{\pi}) \sin (j \theta)$ for $j<0$, and $\chi_{0}(\theta)=1 / \sqrt{2 \pi}$. It will frequently be useful to separate out the operators corresponding to the indices $j=-1,0,1$ from the rest, and we shall often use the notation $L_{\varepsilon}^{\prime}$ to refer to the projection of the operator acting on these three components, and $L_{\varepsilon}^{\prime \prime}$ to refer to the operator acting on all the others together. This division is natural because, by (3.26), the term of order zero in $L_{\varepsilon, j}$ is strictly negative when $|j|>1$, and so the estimates for $L_{\varepsilon}^{\prime \prime}$ follow easily from the maximum principle, but this is false when $|j| \leq 1$ and $\tau$ is small.

\subsection{Jacobi fields.}

A deeper reason for the separation into low and high eigencomponents in the Jacobi operator becomes apparent when one examines the Jacobi fields, i.e. the solutions of $L_{\varepsilon} \phi=0$. Any such function may be expanded into its eigenseries, $\phi=\sum \phi_{j}(s) \chi_{j}(\theta)$, and then each $\phi_{j}$ solves $L_{\varepsilon, j} \phi_{j}=0$. It turns out that the solutions for this problem when $j=0, \pm 1$ may be determined explicitly in terms of the functions $\rho$ or $\sigma$; in fact, these Jacobi fields correspond to quite explicit one-parameter families of CMC surfaces of which $\Sigma_{\varepsilon}$ is an element. To exhibit these, first note that any smooth one-parameter family $\Sigma(\eta)$ of CMC surfaces, with $\Sigma(0)=\Sigma_{\varepsilon}$, will have differential at $\eta=0$ which is a Jacobi field on $\Sigma_{\varepsilon}$. (This is meant in the sense that $\Sigma(\eta)$ should be written, for small $\eta$, as a normal graph over $\Sigma(0)$. This is possible over any fixed compact set of $\Sigma_{\varepsilon}$ for some nontrivial range of values of $\eta$ which might diminish to zero as the compact set grows. However, this is sufficient to make sense of the derivative at $\eta=0$.) The one-parameter families of CMC surfaces here are simple to describe: the first two families, corresponding to the two different solutions of $L_{\varepsilon, 0} \phi=0$, arise from varying the necksize parameter $\varepsilon$, and translating the $t$-variable, i.e. translating along the axis of $\Sigma_{\varepsilon}$. We denote the associated Jacobi fields by $\Psi_{\varepsilon}^{0,-}$ and $\Psi_{\varepsilon}^{0,+}$, respectively. The other families arise from either translating or rotating the axis of $\Sigma_{\varepsilon}$, so that one such translation and one such rotation will correspond to solutions $\Psi_{\varepsilon}^{j,+}$ and $\Psi_{\varepsilon}^{j,-}$ of $L_{\varepsilon, j}$ for $j=1$, 
while the translation and rotation in the orthogonal direction corresponds to solutions for $j=-1$. In fact, we may determine these solutions explicitly in terms of the function $\rho$. Let us write

$$
\Psi_{\varepsilon}^{j, \pm}(t, \theta):=\Phi_{\varepsilon}^{j, \pm}(t) \chi_{j}(\theta)
$$

We obtain :

Proposition 18. The coefficient functions $\Phi_{\varepsilon}^{j, \pm}$ of the Jacobi fields $\Psi_{\varepsilon}^{j, \pm}$ for $\Sigma_{\varepsilon}$ for $j=-1,0,1$, are given by the formula

$$
\begin{aligned}
\Phi_{\varepsilon}^{0,+} & =\rho_{t} / \sqrt{1+\rho_{t}^{2}}, & \Phi_{\varepsilon}^{0,-} & =-\partial_{\varepsilon} \rho / \sqrt{1+\rho_{t}^{2}} \\
\Phi_{\varepsilon}^{1,+} & =\Phi_{\varepsilon}^{-1,+}, & \Phi_{\varepsilon}^{1,-} & =\Phi_{\varepsilon}^{-1,-}, \\
& =-1 / \sqrt{1+\rho_{t}^{2}}, & & =-\left(t+\rho \rho_{t}\right) / \sqrt{1+\rho_{t}^{2}} .
\end{aligned}
$$

Proof. First consider the families given by translations. Suppose that the function $w$ is chosen locally so that its normal graph is a (small) translation of magnitude $d$ of $\Sigma_{\varepsilon}$ along the $x$ axis. Thus, for some value $t^{\prime}$ near to $t$ and some value of $\theta^{\prime}$ near to $\theta$,

$$
\left\{\begin{aligned}
\rho(t) \cos \theta+d & =\rho\left(t^{\prime}\right) \cos \theta^{\prime}-w\left(t^{\prime}, \theta^{\prime}\right) \cos \theta^{\prime} \\
\rho(t) \sin \theta & =\rho\left(t^{\prime}\right) \sin \theta^{\prime}-w\left(t^{\prime}, \theta^{\prime}\right) \sin \theta^{\prime} \\
t & =t^{\prime}+w\left(t^{\prime}, \theta^{\prime}\right) \rho_{t}\left(t^{\prime}\right)
\end{aligned}\right.
$$

This system is equivalent to

$$
\begin{aligned}
\rho^{2}(t)= & \rho^{2}\left(t^{\prime}\right)-2 w\left(t^{\prime}, \theta^{\prime}\right) \rho\left(t^{\prime}\right)+2 d w\left(t^{\prime}, \theta^{\prime}\right) \cos \theta^{\prime} \\
& +w^{2}\left(t^{\prime}, \theta^{\prime}\right)-2 d \rho\left(t^{\prime}\right) \cos \theta^{\prime}+d^{2}
\end{aligned}
$$

and

$$
t=t^{\prime}+w\left(t^{\prime}, \theta^{\prime}\right) \rho_{t}\left(t^{\prime}\right)
$$

After inserting the value of $t$ from this second equation into the first and collecting the lowest order terms we get

$$
w\left(t^{\prime}, \theta^{\prime}\right)=-d \cos \theta^{\prime} /\left(1+\rho_{t}^{2}\left(t^{\prime}\right)\right)+\text { higher order terms. }
$$

Recalling that the normal of $\Sigma_{\varepsilon}$ at $\mathbf{x}_{\varepsilon}\left(t^{\prime}, \theta^{\prime}\right)$ is

$$
\nu\left(t^{\prime}, \theta^{\prime}\right)=\frac{1}{\sqrt{1+\rho_{t}^{2}}}\left(-\cos \theta^{\prime},-\sin \theta^{\prime}, \rho_{t}\left(t^{\prime}\right)\right)
$$


we get the stated expression for $\Phi_{\varepsilon}^{1,+}$. The expression for $\Phi_{\varepsilon}^{-1,+}$, corresponding to translations along the $y$ axis, is derived in an identical manner.

In fact, nearly identical arguments work in all other cases as well. The relevant systems of equations are

$$
\left\{\begin{aligned}
\rho(t) \cos \theta & =\rho\left(t^{\prime}\right) \cos \theta^{\prime}-w\left(t^{\prime}, \theta^{\prime}\right) \cos \theta^{\prime} \\
\rho(t) \sin \theta & =\rho\left(t^{\prime}\right) \sin \theta^{\prime}-w\left(t^{\prime}, \theta^{\prime}\right) \sin \theta^{\prime} \\
t+d & =t^{\prime}+w\left(t^{\prime}, \theta^{\prime}\right) \rho_{t}\left(t^{\prime}\right)
\end{aligned}\right.
$$

for the translation of size $d$ along the $z$ axis,

$$
\left\{\begin{aligned}
\rho_{\varepsilon+d}(t) \cos \theta & =\rho_{\varepsilon}\left(t^{\prime}\right) \cos \theta^{\prime}-w\left(t^{\prime}, \theta^{\prime}\right) \cos \theta^{\prime} \\
\rho_{\varepsilon+d}(t) \sin \theta & =\rho_{\varepsilon}\left(t^{\prime}\right) \sin \theta^{\prime}-w\left(t^{\prime}, \theta^{\prime}\right) \sin \theta^{\prime} \\
t & =t^{\prime}+w\left(t^{\prime}, \theta^{\prime}\right)\left(\rho_{\varepsilon}\right)_{t}\left(t^{\prime}\right)
\end{aligned}\right.
$$

for the variation of necksize, and

$$
\left\{\begin{aligned}
(\cos d) \rho(t) \cos \theta+(\sin d) t & =\rho\left(t^{\prime}\right) \cos \theta^{\prime}-w\left(t^{\prime}, \theta^{\prime}\right) \cos \theta^{\prime} \\
\rho(t) \sin \theta & =\rho\left(t^{\prime}\right) \sin \theta^{\prime}-w\left(t^{\prime}, \theta^{\prime}\right) \sin \theta^{\prime} \\
(\cos d) t-(\sin d) \rho(t) \cos \theta & =t^{\prime}+w\left(t^{\prime}, \theta^{\prime}\right) \rho_{t}\left(t^{\prime}\right),
\end{aligned}\right.
$$

for a rotation of size $d$ of the $z$-axis toward the $x$-axis, and similarly for the rotation toward the $y$-axis.

The calculations proceed as in the first case, and we leave the details to the reader.

Corollary 3. The expressions for these Jacobi fields in terms of the functions $\sigma$ and $k$ and the parameter $\tau$ are

$$
\begin{aligned}
& \Phi_{\varepsilon}^{0,+}=\sigma_{s}, \\
& \Phi_{\varepsilon}^{0,-}=\frac{\sqrt{1-\tau^{2}}}{\tau} \sigma_{s} \partial_{\tau} k-\sqrt{1-\tau^{2}} e^{\sigma} \cosh \sigma\left(1+\tau \partial_{\tau} \sigma\right), \\
& \Phi_{\varepsilon}^{1,+}=\Phi_{\varepsilon}^{-1,+}=-\tau \cosh \sigma \\
& \Phi_{\varepsilon}^{1,-}=\Phi_{\varepsilon}^{-1,-}=-\tau k\left(\cosh \sigma+\sigma_{s} e^{\sigma}\right) .
\end{aligned}
$$

The proof involves simply inserting the expressions for $\rho, t$ and $\varepsilon$ in terms of $\sigma, k$ and $\tau$ into the previous formulæ.

We shall require later the limits of these Jacobi fields as $\varepsilon$ tends to zero. 
Proposition 19. Let $I \subset \mathbb{R}$ be any compact interval. Then the following limits exist uniformly for $s \in I$ :

$$
\begin{gathered}
\lim _{\varepsilon \rightarrow 0} \Phi_{\varepsilon}^{0,+}(\varepsilon s)=\tanh s, \quad \lim _{\varepsilon \rightarrow 0} \Phi_{\varepsilon}^{0,-}(\varepsilon s)=-(1-s \tanh s), \\
\lim _{\varepsilon \rightarrow 0} \Phi_{\varepsilon}^{1,+}(\varepsilon s)=\lim _{\varepsilon \rightarrow 0} \Phi_{\varepsilon}^{-1,+}(\varepsilon s)=-\frac{1}{\cosh s} \\
\lim _{\varepsilon \rightarrow 0} \frac{1}{\varepsilon} \Phi_{\varepsilon}^{1,-}(\varepsilon s)=\lim _{\varepsilon \rightarrow 0} \frac{1}{\varepsilon} \Phi_{\varepsilon}^{-1,-}(\varepsilon s)=-\left(\frac{s}{\cosh s}+\sinh s\right) .
\end{gathered}
$$

Proof. We have used the variable $s$ in the statement of the theorem because by Proposition 10, $\varepsilon s / t \rightarrow 1$ uniformly for $s \in I$. The limits may be calculated using either the estimates for $\rho$ from Proposition 9, or else the expressions for these Jacobi fields from Corollary 3 in terms of $\sigma$, and then using the limiting behaviour of $\sigma$ as determined in Corollary 1 .

The Jacobi fields we have considered so far, $\Psi_{\varepsilon}^{j, \pm}, j=0, \pm 1$, are all either bounded (in fact periodic) or linearly growing (because both $k$ and $\partial_{\tau} k$ are linearly growing). There are of course, two linearly independent solutions of the equation $L_{\varepsilon, j} \phi=0$ for all $j$ with $|j|>1$ as well. It is proved in [9], following [13], that there exists a discrete sequence of positive numbers $\gamma_{j} \rightarrow \infty,|j|>1$, with $\gamma_{-j}=\gamma_{j}$, and for each $j$ a solution $\Phi_{\varepsilon}^{j, \pm}$ of $L_{\varepsilon, j} \phi=0$ such that

$$
e^{ \pm \gamma_{j} s} \Phi_{\varepsilon}^{j, \pm}
$$

are periodic functions of $s$. In particular,

$$
\left|\Phi_{\varepsilon}^{j,+}\right| \leq c e^{-\gamma_{j} s}, \quad\left|\Phi_{\varepsilon}^{j,-}\right| \leq c e^{\gamma_{j} s}, \quad \text { for all } s \in \mathbb{R} .
$$

In fact, $\Phi_{\varepsilon}^{j,-}(s)=\Phi_{\varepsilon}^{j,+}(-s)$. Because of Corollary 3 , it is natural to define $\gamma_{0}=\gamma_{ \pm 1}=0$.

While there are analogous conclusions were we to be using the independent variable $t$ instead of $s$, the values of these 'indicial exponents' $\gamma_{j}=\gamma_{j}(\varepsilon)$ would behave in a less desirable way as $\varepsilon$ tends to zero.

Proposition 20. For any $\eta>0$, there exists an $\varepsilon_{0}>0$ such that when $\varepsilon \in\left(0, \varepsilon_{0}\right)$, the numbers $\gamma_{j}$ satisfy $\gamma_{j} \geq \sqrt{4-\eta}$ for $|j|>1$.

Proof. The $\Phi_{\varepsilon}^{j, \pm}$ are homogeneous solutions for the ordinary differential operator $-\partial_{s s}^{2}+Q_{j}$, where $Q_{j}=-\tau^{2} \cosh (2 \sigma)+j^{2}$. We are trying to estimate 
the exponential growth rate of solutions for this operator. It follows from (3.26) that

$$
\tau^{2} \leq \tau^{2} \cosh (2 \sigma) \leq 2-\tau^{2} .
$$

Thus, we see that $Q_{j} \geq j^{2}-2+\tau^{2}$. In particular, for $|j| \geq 3, Q_{j}>4$. In this case, the result is clear: $e^{ \pm 2 s}$ are supersolutions for the operator, and homogeneous solutions bounded by these supersolutions may be constructed by the shooting method, as described next for the slightly more complicated case when $j= \pm 2$.

Let $w$ be the unique decreasing solution of $\left(-\partial_{s s}^{2}+Q_{2}\right) w=0$ with $w(0)=1$. This solution may be constructed as a limit, as $s_{1} \rightarrow \infty$, of solutions $w_{s_{1}}$ of this equation on $\left[0, s_{1}\right]$ with $w_{s_{1}}(0)=1, w_{s_{1}}\left(s_{1}\right)=0$. Since $e^{-\sqrt{2+\tau^{2}} s}$ is a supersolution for this operator and dominates $w_{s_{1}}$ at the endpoints, $s=0$ and $s=s_{1}$, it gives an upper bound for $w_{s_{1}}$ on the whole interval $0 \leq s \leq s_{1}$. Thus the limit as $s_{1} \rightarrow \infty$, which we call $w$, exists and is also bounded by this same function.

Now, using Proposition 13, we can choose $s_{0}$ sufficiently large so that $2 \tau^{2} \cosh ^{2} \sigma \leq \eta / 2$ on $\left[s_{0}, S_{\varepsilon} / 2-s_{0}\right]$ for all $\tau$ small enough, say $\tau \leq \tau_{0}$.

For simplicity in the notations, we set $S_{0}=S_{\varepsilon} / 2-s_{0}$ and $\beta:=\sqrt{4-\eta / 2}$. We choose $a, b \in \mathbb{R}$ such that $\bar{w}:=a e^{\beta s}+b e^{-\beta s}$ satisfies $\bar{w}\left(s_{0}\right)=w\left(s_{0}\right)$ and $\bar{w}\left(S_{0}\right)=w\left(S_{0}\right)$. Explicitly, we have

$$
a=\frac{w\left(S_{0}\right) e^{\beta S_{0}}-w\left(s_{0}\right) e^{\beta s_{0}}}{e^{2 \beta S_{0}}-e^{2 \beta s_{0}}} \quad \text { and } \quad b=\frac{w\left(s_{0}\right) e^{-\beta s_{0}}-w\left(S_{0}\right) e^{-\beta S_{0}}}{e^{-2 \beta s_{0}}-e^{-2 \beta S_{0}}} .
$$

Thanks to the fact that $Q_{2}>\beta^{2}$ over $\left[s_{0}, S_{0}\right]$, we may use the maximum principle to prove that $w \leq \bar{w}$ on $\left[s_{0}, S_{0}\right]$. We are going to show that

$$
w\left(S_{0}\right) \leq 2 w\left(s_{0}\right) e^{-\beta\left(S_{0}-s_{0}\right)} .
$$

The function $w$ being strictly decreasing, we have $w\left(S_{0}\right)<w\left(s_{0}\right)$ and thus we find that $b>0$. We may as well assume that $w\left(S_{0}\right)>$ $w\left(s_{0}\right) e^{-\beta\left(S_{0}-s_{0}\right)}$, otherwise there is nothing to prove. Under such an assumption $a>0$ and thus $b \in\left(0, w\left(s_{0}\right) e^{\beta s_{0}}\right)$ (since $\bar{w}\left(s_{0}\right)=w\left(s_{0}\right)$ ).

Still using the fact that $w$ is strictly decreasing we find that

$$
w\left(S_{0}\right) \leq \min _{s \in\left[s_{0}, S_{0}\right]} \bar{w} .
$$

But, the infimum of $\bar{w}$ over $\mathbb{R}$ is achieved at the point $s_{m} \geq 0$ which satisfies $e^{2 \beta s_{m}}=b / a$. First we rule out the case $s_{m}<S_{0}$. Indeed, in this case 
$w\left(S_{0}\right)<\bar{w}\left(s_{m}\right)=2 \sqrt{a b}$. Introducing in this inequality the expressions for both $a$ and $b$, we find that

$$
\cosh ^{2}\left(\beta\left(S_{0}-s_{0}\right)\right) w^{2}\left(S_{0}\right)-2 \cosh \left(\beta\left(S_{0}-s_{0}\right)\right) w\left(S_{0}\right) w\left(s_{0}\right)+w^{2}\left(s_{0}\right)<0,
$$

which is not possible. Therefore, we always have $s_{m} \geq S_{0}$ and this implies that

$$
a=b e^{-2 \beta s_{m}} \leq b e^{-2 \beta S_{0}} .
$$

In particular we obtain (since $b<w\left(s_{0}\right) e^{\beta s_{0}}$ )

$$
w\left(S_{0}\right) \leq \bar{w}\left(S_{0}\right)=a e^{\beta S_{0}}+b e^{-\beta S_{0}} \leq 2 b e^{-\beta S_{0}} \leq 2 w\left(s_{0}\right) e^{-\beta\left(S_{0}-s_{0}\right)} .
$$

In every case, we conclude that $w\left(S_{0}\right) \leq 2 w\left(s_{0}\right) e^{-\beta\left(S_{0}-s_{0}\right)}$, where we recall that $S_{0}=S_{\varepsilon} / 2-s_{0}$. Furthermore, $w$ is monotone decreasing, so its maximum on any interval is attained at the left endpoint and its minimum at the right endpoint. Thus $w\left(S_{\varepsilon} / 2+s_{0}\right) \leq 2 w\left(s_{0}\right) e^{2 \beta s_{0}} e^{-\beta S_{\varepsilon} / 2}$. Reducing $\varepsilon$ is necessary, we may assume that

$$
2 e^{2 \beta s_{0}} e^{-\beta S_{\varepsilon} / 2} \leq e^{-\tilde{\beta} S_{\varepsilon} / 2},
$$

were we have set $\tilde{\beta}=\sqrt{4-\eta}$.

In order to finish the proof, note that

$$
w\left(s+S_{\varepsilon} / 2\right)=\frac{w\left(s_{0}+S_{\varepsilon} / 2\right)}{w\left(s_{0}\right)} w(s) \quad \text { for all } s .
$$

To see this, simply observe that $W(s):=w\left(s_{0}+S_{\varepsilon} / 2\right) w(s)-w\left(s+S_{\varepsilon} / 2\right) w\left(s_{0}\right)$ solves $\left(-\partial_{s s}^{2}+Q_{2}\right) W=0$, decays exponentially at $\infty$ and takes the value 0 at $s_{0}$, hence by the maximum principle must vanish identically.

Hence for all $N \geq 1$, we have $w\left(N S_{\varepsilon} / 2+s_{0}\right) \leq w\left(s_{0}\right) e^{-N \tilde{\beta} S_{\varepsilon} / 2}$. The conclusion of the Lemma now follows at once.

\subsection{Mapping properties of the Jacobi operator.}

To fully analyze the problem (4.7), we must study the mapping properties of the Jacobi operator $L_{\varepsilon}$, both for fixed $\varepsilon>0$ and uniformly down to $\varepsilon=0$. To state this result it is first necessary to define appropriate function spaces on which the Jacobi operator will act; these are exponentially weighted Hölder spaces. This is one of the main places where the difference between the independent variables $s$ and $t$ is seen: it is possible to obtain good 
mapping properties on spaces of this type, defined either in terms of the $s$ or $t$ variables, for fixed $\varepsilon$, but it is much less pleasant to obtain the uniform. behaviour down to $\varepsilon=0$ when using $t$. Fortunately, this uniformity does occur when using $s$, and so from now on, unless saying explicitly otherwise, this choice of independent variable will be used in the sequel.

The definition of the weighted Hölder spaces is the natural one :

Definition 1. Parametrize $\mathbb{R} \times S^{1}$ by the variables $(s, \theta)$. For each $r \in \mathbb{N}$ and $0<\alpha<1$ and $s \in \mathbb{R}$, let

$$
|w|_{r, \alpha,[s, s+1]}
$$

denote the usual $\mathcal{C}^{r, \alpha}$ Hölder norm on the set $[s, s+1] \times S^{1}$. Then for any $\mu \in \mathbb{R}$ and $s_{0} \in \mathbb{R}$,

$$
\begin{aligned}
\mathcal{C}_{\mu}^{r, \alpha}\left(\left[s_{0},+\infty\right) \times S^{1}\right)=\left\{w \in \mathcal{C}_{\text {loc }}^{r, \alpha}\left(\left[s_{0},+\infty\right) \times S^{1}\right)\right. \text { and } \\
\left.\|w\|_{r, \alpha, \mu}=\sup _{s \geq s_{0}} e^{-\mu s}|w|_{r, \alpha,[s, s+1]}<\infty\right\} .
\end{aligned}
$$

In particular, the function $e^{\mu s}$ is in $\mathcal{C}_{\mu}^{r, \alpha}\left(\left[s_{0},+\infty\right) \times S^{1}\right)$.

Recall now the splitting of $L_{\varepsilon}$ into $L_{\varepsilon}^{\prime}$ and $L_{\varepsilon}^{\prime \prime}$, corresponding to the operator induced on the eigenspaces with $|j| \leq 1$ and $|j|>1$, respectively. $\Pi^{\prime}$ and $\Pi^{\prime \prime}$ are the projectors onto the corresponding subspaces. This will often be abbreviated by letting $\Pi^{\prime} w=w^{\prime}$, and so on. The main result of this section is the

Proposition 21. Fix $\mu$ with $\mu \in(1,2)$. Then there exists an $\varepsilon_{0}>0$, depending only on $\mu$, such that whenever $\varepsilon \in\left(0, \varepsilon_{0}\right)$, there exists a unique solution $w \in \mathcal{C}_{-\mu}^{2, \alpha}\left(\left[S_{\varepsilon} / 8, \infty\right) \times S^{1}\right)$ of the problem

$$
\begin{cases}L_{\varepsilon} w=f & \text { in } \quad\left(S_{\varepsilon} / 8, \infty\right) \times S^{1} \\ \Pi^{\prime \prime} w=\phi^{\prime \prime} & \text { on } \quad\left\{S_{\varepsilon} / 8\right\} \times S^{1},\end{cases}
$$

for $f \in \mathcal{C}_{-\mu}^{0, \alpha}\left(\left[S_{\varepsilon} / 8, \infty\right) \times S^{1}\right)$ and $\phi^{\prime \prime} \in \Pi^{\prime \prime}\left(\mathcal{C}^{2, \alpha}\left(S^{1}\right)\right)$. The solution of the homogeneous Dirichlet problem, when $\phi^{\prime \prime}=0$, will be denoted $w=G_{\varepsilon}(f)$, while the Poisson operator, which gives the solution when $f=0$, will be denoted by $w=P_{\varepsilon}\left(\phi^{\prime \prime}\right)$. The linear maps

$$
\begin{aligned}
G_{\varepsilon} & : \mathcal{C}_{-\mu}^{0, \alpha}\left(\left[S_{\varepsilon} / 8, \infty\right) \times S^{1}\right) \\
\varepsilon^{\mu / 4} P_{\varepsilon}: \Pi^{\prime \prime}\left(\mathcal{C}^{2, \alpha}\left(S^{1}\right)\right) & \longrightarrow \mathcal{C}_{-\mu}^{2, \alpha}\left(\left[S_{\varepsilon} / 8, \infty\right) \times S^{1}\right)
\end{aligned}
$$

are bounded uniformly for all $\varepsilon \in\left(0, \varepsilon_{0}\right)$. 
Proof. The proof of the existence of $G_{\varepsilon}$ and $P_{\varepsilon}$ and of their uniformity is accomplished in a number of steps. Solutions are constructed on each eigenspace of the Laplacian on $S^{1}$, and the cases where $|j| \leq 1$ must be treated somewhat differently than the others.

We shall give the proof of this result in a slightly more general context where the boundary point $S_{\varepsilon} / 8$ is replaced by $s_{0}$ arbitrarily chosen in $\mathbb{R}$.

Fix $f$ and $\phi$ in the appropriate function spaces. We decompose $w=$ $w^{\prime}+w^{\prime \prime}, f=f^{\prime}+f^{\prime \prime}$, then we must solve

$$
\left\{\begin{aligned}
L_{\varepsilon}^{\prime} w^{\prime}=f^{\prime} & \text { for } s>s_{0} \\
L_{\varepsilon}^{\prime \prime} w^{\prime \prime}=f^{\prime \prime} & \text { for } s>s_{0} \\
w^{\prime \prime}=\phi^{\prime \prime} & \text { for } s=s_{0} .
\end{aligned}\right.
$$

Notice that no boundary conditions are imposed on $w^{\prime}$ at $s=s_{0}$. We will also need to decompose

$$
w^{\prime}(s, \theta)=\frac{1}{\sqrt{\pi}} w_{-1}(s) \sin \theta+\frac{1}{\sqrt{2 \pi}} w_{0}(s)+\frac{1}{\sqrt{\pi}} w_{1}(s) \cos \theta,
$$

and

$$
f^{\prime}(s, \theta)=\frac{1}{\sqrt{\pi}} f_{-1}(s) \sin \theta+\frac{1}{\sqrt{2 \pi}} f_{0}(s)+\frac{1}{\sqrt{\pi}} f_{1}(s) \cos \theta .
$$

Step 1: We first consider the problem where $\phi^{\prime \prime}=0$. Thus $f \in \mathcal{C}_{-\mu}^{0, \alpha}$, and multiplying by a suitable factor, we may assume that

$$
\left\|f^{\prime}\right\|_{0, \alpha,-\mu}+\left\|f^{\prime \prime}\right\|_{0, \alpha,-\mu}=1 .
$$

In this step, we only consider the restriction of the problem to the high eigencomponents. We first show that for every $s_{1}>s_{0}$ there is a unique solution of

$$
\left\{\begin{array}{rlll}
L_{\varepsilon}^{\prime \prime} w_{*}^{\prime \prime}=f^{\prime \prime} & \text { in } & \left(s_{0}, s_{1}\right) \times S^{1} \\
w_{*}^{\prime \prime}=0 & \text { on } & \left\{s_{0}\right\} \times S^{1} \\
w_{*}^{\prime \prime}=0 & \text { on } & \left\{s_{1}\right\} \times S^{1}
\end{array}\right.
$$

The existence of $w_{*}^{\prime \prime}$ follows from a standard variational argument using the energy functional

$$
\mathcal{E}(w)=\int_{s_{0}}^{s_{1}} \int_{S^{1}}\left(\left|\partial_{s} w\right|^{2}+\left|\partial_{\theta} w\right|^{2}-\tau^{2} \cosh (2 \sigma)|w|^{2}+f^{\prime \prime} w\right) d s d \theta
$$


Using the fact that $\forall|j|>1$, we have $j^{2}-\tau^{2} \cosh (2 \sigma)>2$, we see that when we restrict the domain of $\mathcal{E}$ to the span of the eigenfunctions $\chi_{j}(\theta)$ with $|j|>1$, this functional is convex and proper, and the existence of a unique minimizer for it, which we denote by $w_{*}^{\prime \prime}$, is then immediate.

We claim that there exists a constant $\varepsilon_{0}>0$ and a constant $C=C(\mu)>$ 0 , independent of $s_{0}<s_{1}$ and $\varepsilon \in\left(0, \varepsilon_{0}\right)$, such that

$$
\sup _{\theta \in S^{1}} \sup _{s \in\left[s_{0}, s_{1}\right]} e^{\mu s}\left|w_{*}^{\prime \prime}(s, \theta)\right| \leq C(\mu) .
$$

Assuming that the claim is already proven, we can choose a sequence $s_{1, i}$ tending to $+\infty$ and build $w_{*, i}^{\prime \prime}$ the corresponding solutions of (4.11). The uniform bound above allows us to extract from the sequence $w_{*, i}^{\prime \prime}$ a subsequence which converges to a solution $w^{\prime \prime}$ of

$$
\left\{\begin{array}{rlll}
L_{\varepsilon}^{\prime \prime} w^{\prime \prime}=f^{\prime \prime} & \text { in } \quad\left(s_{0},+\infty\right) \times S^{1} \\
w^{\prime \prime}=0 & \text { on } \quad\left\{s_{0}\right\} \times S^{1},
\end{array}\right.
$$

which satisfies

$$
\sup _{\theta \in S^{1}} \sup _{s \in\left[s_{0}, s_{1}\right]} e^{\mu s}\left|w^{\prime \prime}\right| \leq C(\mu) .
$$

and then, by classical elliptic estimates, that

$$
\left\|w^{\prime \prime}\right\|_{2, \alpha,-\mu} \leq c(\mu)
$$

for some constant $c(\mu)>0$ independent of $s_{0} \in \mathbb{R}$ and $\varepsilon \in\left(0, \varepsilon_{0}\right)$. The claim is proved by contradiction. By assumption, we have $e^{\mu s}\left|f^{\prime \prime}(s, \theta)\right| \leq 1$ for $s_{0} \leq s \leq s_{1}, \theta \in S^{1}$. If the assertion were not true, then there would exist sequences of numbers $s_{0, i}, s_{1, i}$, functions $f_{i}^{\prime \prime}$, Delaunay parameters $\varepsilon_{i}$ and corresponding solutions $w_{*, i}^{\prime \prime}$ such that

$$
A_{i}:=\sup _{\theta \in S^{1}} \sup _{s_{0, i} \leq s \leq s_{1, i}} e^{\mu s}\left|w_{*, i}^{\prime \prime}\right| \longrightarrow \infty,
$$

and

$$
\sup _{\theta \in S^{1}} \sup _{s_{0, i} \leq s \leq s_{1, i}} e^{\mu s}\left|f_{i}^{\prime \prime}\right| \leq 1
$$

Suppose that this maximum, for each $i$, is attained at some point $\left(s_{i}, \theta_{i}\right)$, and define

$$
\tilde{w}_{i}^{\prime \prime}(s, \theta)=A_{i}^{-1} e^{\mu s_{i}} w_{s_{1, i}}^{\prime \prime}\left(s+s_{i}, \theta\right), \quad \tilde{f}_{i}^{\prime \prime}(s, \theta)=A_{i}^{-1} e^{\mu s_{i}} f^{\prime \prime}\left(s+s_{i}, \theta\right) .
$$


Then

$$
\sup _{\theta \in S^{1}} \sup _{s_{0, i}-s_{i} \leq s \leq s_{1, i}-s_{i}} e^{\mu s}\left|\tilde{w}_{i}^{\prime \prime}\right|=1,
$$

and this supremum is attained on $\{0\} \times S^{1}$, while $\tilde{f}_{i}^{\prime \prime} \rightarrow 0$ in norm. Furthermore,

$$
L_{\varepsilon}^{\prime \prime} \tilde{w}_{i}^{\prime \prime}=\partial_{s s}^{2} \tilde{w}_{i}^{\prime \prime}+\partial_{\theta \theta}^{2} \tilde{w}_{i}^{\prime \prime}+\tau_{i}^{2} \cosh \left(2 \sigma_{i}\left(\cdot+s_{i}\right)\right) \tilde{w}_{i}^{\prime \prime}=\tilde{f}_{i}^{\prime \prime}
$$

on $\left[s_{0, i}-s_{i}, s_{1, i}-s_{i}\right] \times S^{1}$.

Passing to a subsequence if necessary, we assume that $s_{0, i}-s_{i}$ converges to $v_{1} \in \mathbb{R} \cup\{-\infty\}$ and $s_{1, i}-s_{i}$ converges to $v_{2} \in \mathbb{R} \cup\{+\infty\}$. By using the result of Proposition 12, the bounds above, as well as those provided by elliptic estimates, we can also assume that $\tilde{w}_{i}^{\prime \prime}$ converges, along with all its derivatives, over any compact subset of $\left(v_{1}, v_{2}\right) \times S^{1}$ (including endpoints if either is finite) to a function $\tilde{w}^{\prime \prime}$, which satisfies $e^{\mu s}\left|\tilde{w}^{\prime \prime}\right| \leq 1$ over this set, is nonvanishing (because of the normalization of $\tilde{w}_{i}^{\prime \prime}$ at $s=0$ ), and which solves one of the following equations:

$$
\partial_{s s}^{2} \tilde{w}^{\prime \prime}+\partial_{\theta \theta}^{2} \tilde{w}^{\prime \prime}+\tau^{2} \cosh (2 \sigma(s+\bar{s})) \tilde{w}^{\prime \prime}=0,
$$

for some $\varepsilon \in\left(0, \varepsilon_{0}\right)$ and $\bar{s} \in \mathbb{R}$,

$$
\partial_{s s}^{2} \tilde{w}^{\prime \prime}+\partial_{\theta \theta}^{2} \tilde{w}^{\prime \prime}=0
$$

or

$$
\partial_{s s}^{2} \tilde{w}^{\prime \prime}+\partial_{\theta \theta}^{2} \tilde{w}^{\prime \prime}+\frac{2}{\cosh ^{2}(s+\bar{s})} \tilde{w}^{\prime \prime}=0, \quad \text { for some } \quad \bar{s} \in \mathbb{R},
$$

on $\left[v_{1}, v_{2}\right] \times S^{1}$. In addition, if either $v_{1}$ or $v_{2}$ is finite, then $\tilde{w}^{\prime \prime}$ vanishes at that endpoint.

We must analyze a few cases, depending on the values of $v_{1}$ and $v_{2}$ and which of the equations above is satisfied by $\tilde{w}^{\prime \prime}$. The goal in each case is to show that $\tilde{w}^{\prime \prime}$ must, in fact, vanish identically, which would be a contradiction.

The point, in all cases, is that we wish to multiply the appropriate equation for $\tilde{w}^{\prime \prime}$ by $\tilde{w}^{\prime \prime}$ and integrate by parts, to obtain

$$
\begin{gathered}
\int_{v_{1}}^{v_{2}} \int_{S^{1}}\left|\partial_{s} \tilde{w}^{\prime \prime}\right|^{2}+\left|\partial_{\theta} \tilde{w}^{\prime \prime}\right|^{2}-\tau^{2} \cosh (2 \sigma(s+\bar{s}))\left|\tilde{w}^{\prime \prime}\right|^{2} d s d \theta=0 \\
\int_{v_{1}}^{v_{2}} \int_{S^{1}}\left|\partial_{s} \tilde{w}^{\prime \prime}\right|^{2}+\left|\partial_{\theta} \tilde{w}^{\prime \prime}\right|^{2} d s d \theta=0
\end{gathered}
$$


or

$$
\int_{v_{1}}^{v_{2}} \int_{S^{1}}\left|\partial_{s} \tilde{w}^{\prime \prime}\right|^{2}+\left|\partial_{\theta} \tilde{w}^{\prime \prime}\right|^{2}-\frac{2}{\cosh ^{2}(s+\bar{s})}\left|\tilde{w}^{\prime \prime}\right|^{2} d s d \theta=0 .
$$

In each of these three cases we see that the integrand is positive, because we always have the inequality

$$
\int_{v_{1}}^{v_{2}}\left|\partial_{\theta} \tilde{w}^{\prime \prime}\right|^{2} d s \geq 4 \int_{v_{1}}^{v_{2}}\left|\tilde{w}^{\prime \prime}\right|^{2} d s
$$

and so we would conclude that $\tilde{w}^{\prime \prime} \equiv 0$, which is a contradiction.

To make this argument work, it suffices to show that the boundary terms in the integration by parts vanish. When either $v_{1}$ or $v_{2}$ is finite, this is immediate from the Dirichlet conditions at that boundary, so it remains to show that if either $v_{1}$ or $v_{2}$ is infinite, then $\tilde{w}^{\prime \prime}$ decays exponentially in that direction. Any unbounded solution of (4.13) on a half-line must grow at least at the rate $e^{2|s|}$, which would violate the condition $e^{\mu s}\left|\tilde{w}^{\prime \prime}\right| \leq 1$, so we see that $\tilde{w}^{\prime \prime}$ must decrease exponentially in this case. The same argument works when $\tilde{w}^{\prime \prime}$ satisfies (4.14) because solutions of that equation have the same asymptotic rates of growth or decay as solutions of (4.13). Finally, if $\tilde{w}^{\prime \prime}$ satisfies (4.12), we first choose $\eta>0$ such that $\sqrt{4-\eta}>\mu$, then, we apply Proposition 20, which states that any unbounded solution must grow at least at the rate $e^{\sqrt{4-\eta}|s|}$ provided $\varepsilon$ is less than, say, $\varepsilon_{0}$. Therefore, we can eliminate the possibility of exponential growth. This ends the proof of the claim.

Step 2: We now consider the cases when $|j| \leq 1$. The argument when $j= \pm 1$ is almost identical to the one for $j=0$, so we shall just consider the latter case, commenting on the end on the very minor changes that need to be made. Thus, recalling that we are no longer requiring any boundary conditions, we wish to find a solution to the problem

$$
L_{\varepsilon, 0} w_{0}:=\partial_{s s}^{2} w_{0}+\tau^{2} \cosh (2 \sigma) w_{0}=f_{0} \quad \text { in } \quad\left[s_{0}, \infty\right),
$$

with the desired decay property at infinity. We find this solution again as a limit of functions $w_{*}$ solutions of $L_{\varepsilon, 0} w_{*}=f_{0}$ on $\left[s_{0}, s_{1}\right)$, where now $w_{*}\left(s_{1}\right)=\partial_{s} w_{*}\left(s_{1}\right)=0$. For convenience, we choose a $\mathcal{C}^{0, \alpha}$ extension of $f_{0}$, vanishing when $s<s_{0}-1$, say, and consider the solution $w_{*}$ for this extended right hand side, now defined on $\left(-\infty, s_{1}\right]$.

As in Step 1, we claim that there exists a constant $C=C(\mu)$, independent of $s_{0}, s_{1}$ and $\varepsilon$, such that

$$
\sup _{s \in\left(-\infty, s_{1}\right]} e^{\mu s}\left|w_{*}\right| \leq C .
$$


Once this claim is proved, the arguments of the proof are identical to those in Step 1, so we shall omit them.

Again this is proved by contradiction. First, note that when $s<s_{0}-1$, $w_{s_{1}}$ is a linear combination of the Jacobi fields $\Phi_{\varepsilon}^{0, \pm}$, hence is at most linearly growing. If the assertion were false, there would exist sequences $f_{0, i}, s_{0, i}$, $s_{1, i}, \varepsilon_{i}$, and $w_{*, i}$ such that

$$
A_{i}:=\sup _{s \in\left(-\infty, s_{1, i}\right]} e^{\mu s}\left|w_{*, i}\right| \longrightarrow \infty
$$

and

$$
\sup _{s \in\left(-\infty, s_{1, i}\right]} e^{\mu s}\left|f_{0, i}\right| \leq 1 .
$$

If this maximum is attained at $\left(s_{i}, \theta_{i}\right), s_{i} \in\left(-\infty, s_{1, i}\right)$, then we rescale the functions and translate the independent variable by $s_{i}$ to obtain a solution of

$$
\frac{d^{2} \tilde{w}_{i}}{d s^{2}}+\tau_{i}^{2} \cosh \left(2 \sigma_{i}\right) \tilde{w}_{i}=\tilde{f}_{0, i}
$$

in $\left(-\infty, s_{1, i}-s_{i}\right]$ which satisfies

$$
\sup _{s \in\left(-\infty, s_{1, i}-s_{i}\right]} e^{\mu s}\left|\tilde{w}_{i}\right|=1
$$

while $\tilde{f}_{0, i}$ tends to zero in norm.

Passing to a subsequence, we obtain in the limit a nontrivial solution $\tilde{w}$ of one the following equations:

$$
\begin{gathered}
\frac{d^{2} \tilde{w}}{d s^{2}}+\tau^{2} \cosh (2 \sigma(s+\bar{s})) \tilde{w}=0, \quad \text { for some } \varepsilon \in\left(0, \varepsilon_{0}\right) \text { and } \bar{s} \in \mathbb{R}, \\
\frac{d^{2} \tilde{w}}{d s^{2}}+\frac{2}{\cosh ^{2}(s+\bar{s})} \tilde{w}=0, \quad \text { for some } \quad \bar{s} \in \mathbb{R}
\end{gathered}
$$

or

$$
\frac{d^{2} \tilde{w}}{d s^{2}}=0
$$

over some interval $(-\infty, v]$, and in each case, $|\tilde{w}| \leq e^{-\mu s}$ in $(-\infty, v]$.

Clearly $v$ cannot be finite, because if it were then $\tilde{w}$ would have to satisfy $\tilde{w}(v)=\partial_{s} \tilde{w}(v)=0$, which would imply that it would vanish identically. 
Now, for each of the three equations we know that there are no exponentially decreasing solutions; for the second and third equations this is obvious, while for the first it follows because we know the family of solutions explicitly. However, since we know that $\tilde{w}$ does decay exponentially as $s \rightarrow \infty$, we again would have to conclude that it vanishes identically, and this is a contradiction.

When $j= \pm 1$, the changes that need to be made in this argument are minor. For example, when $j=1$, for all $s_{1}>s_{0}$, the solution $w_{*}$ is defined as before to be the solution of

$$
L_{\varepsilon, 1} w_{*}:=\partial_{s s}^{2} w_{*}-w_{*}+\tau^{2} \cosh (2 \sigma) w_{*}=f_{1} \quad \text { in } \quad\left[s_{0}, s_{1}\right),
$$

which satisfies $w_{*}\left(s_{1}\right)=\partial_{s} w_{*}\left(s_{1}\right)=0$ and where $f_{1}$ has been extended by 0 in $\left(-\infty, s_{0}-1\right]$.

And to establish its uniform bound, we proceed by contradiction. In this case, however, the limiting equations are now

$$
\begin{gathered}
\frac{d^{2} \tilde{w}}{d s^{2}}-\tilde{w}+\tau^{2} \cosh (2 \sigma(s+\bar{s})) \tilde{w}=0, \quad \text { for some } \quad \varepsilon \in\left(0, \varepsilon_{0}\right) \text { and } \bar{s} \in \mathbb{R}, \\
\frac{d^{2} \tilde{w}}{d s^{2}}-\tilde{w}+\frac{2}{\cosh ^{2}(s+\bar{s})} \tilde{w}=0, \quad \text { for some } \quad \bar{s} \in \mathbb{R},
\end{gathered}
$$

or

$$
\frac{d^{2} \tilde{w}}{d s^{2}}-\tilde{w}=0
$$

on $(-\infty, v] \times S^{1}$, with boundary condition $\tilde{w}(v)=\partial_{s} \tilde{w}(v)=0$ if $v$ is finite, and where $|\tilde{w}| \leq e^{-\mu s}$ for all $s \in(-\infty, v]$.

Once again, $v$ cannot be finite, but now the equations do admit exponentially decreasing solutions at $\pm \infty$. However, all such solutions decay no faster than $e^{-s}$, whereas we have assumed that $\mu \in(1,2)$, so once again we obtain a contradiction.

Step 3: Finally consider the problem when $f=0$ and $\phi^{\prime \prime} \neq 0$. We may as well assume that $\left\|\phi^{\prime \prime}\right\|_{2, \alpha}=1$. Let $\eta(s)$ be a smooth cutoff function equal to 1 for $s \leq 0$ and vanishing for $s \geq 1$. Then

$$
L_{\varepsilon}^{\prime \prime} w=0, \quad w\left(s_{0}, \theta\right)=\phi^{\prime \prime}(\theta)
$$

is equivalent to

$$
L_{\varepsilon}^{\prime \prime} \bar{w}=-L_{\varepsilon}^{\prime \prime}\left(\eta\left(s-s_{0}\right) \phi^{\prime \prime}(\theta)\right), \quad w\left(s_{0}, \theta\right)=0,
$$


which has already been solved in Step 1. Moreover, since

$$
\left\|\eta \phi^{\prime \prime}\right\|_{0, \alpha,-\mu} \leq c e^{\mu s_{0}}
$$

it follows from Step 1 that

$$
\|w\|_{2, \alpha,-\mu} \leq c e^{\mu s_{0}}
$$

as we wished. This completes the proof in all cases.

Corollary 4. Fix $\mu \in(1,2)$. Then there exists a constant $c>0$ and an $\varepsilon_{0}>0$, depending only on $\mu$, such that for $\varepsilon \in\left(0, \varepsilon_{0}\right)$, we have

$$
\left\|\left(P_{\varepsilon}-P_{0}\right)\left(\phi^{\prime \prime}\right)\right\|_{2, \alpha,-\mu} \leq c \varepsilon^{-\mu / 4}\left(\varepsilon^{1 / 2}+\varepsilon^{(6-3 \mu) / 4}\right)\left\|\phi^{\prime \prime}\right\|_{2, \alpha} .
$$

Here, if $\phi^{\prime \prime} \in \Pi^{\prime \prime}\left(\mathcal{C}^{2, \alpha}\left(S^{1}\right)\right)$, the function $P_{0}\left(\phi^{\prime \prime}\right)$ is the unique solution in $\mathcal{C}_{-2}^{2, \alpha}\left(\left[S_{\varepsilon} / 8, \infty\right) \times S^{1}\right)$ of the problem

$$
\left\{\begin{array}{rlll}
\Delta w & =0 & \text { in } & {\left[S_{\varepsilon} / 8, \infty\right) \times S^{1}} \\
w & =\phi^{\prime \prime} & \text { on } & \left\{S_{\varepsilon} / 8\right\} \times S^{1} .
\end{array}\right.
$$

Proof. Write $w_{\varepsilon}=P_{\varepsilon} \phi^{\prime \prime}$ and $w_{0}=P_{0} \phi^{\prime \prime}$. If $w_{\varepsilon}=w_{0}+h$, then $L_{\varepsilon} h=$ $-\left(\tau^{2} \cosh (2 \sigma)\right) w_{0}$ and $\Pi^{\prime \prime} h\left(S_{\varepsilon} / 8, \theta\right)=0$, and so $h=-G_{\varepsilon}\left(\tau^{2} \cosh (2 \sigma) w_{0}\right)$. We first estimate

$$
\|h\|_{2, \alpha,-\mu} \leq c\left\|\tau^{2} \cosh (2 \sigma) w_{0}\right\|_{0, \alpha,-\mu} .
$$

Using

$$
\left\|w_{0}\right\|_{0, \alpha,[s, s+1]} \leq c e^{-2\left(s-S_{\varepsilon} / 8\right)}\left\|\phi^{\prime \prime}\right\|_{0, \alpha} \leq c \varepsilon^{-1 / 2} e^{-2 s}\left\|\phi^{\prime \prime}\right\|_{0, \alpha},
$$

we bound this by

$$
c \varepsilon^{-1 / 2}\left(\sup _{s \geq S_{\varepsilon} / 8} e^{(\mu-2) s}\left\|\tau^{2} \cosh 2 \sigma\right\|_{0, \alpha,[s, s+1]}\right)\left\|\phi^{\prime \prime}\right\|_{0, \alpha} .
$$

When $S_{\varepsilon} / 8 \leq s \leq 3 S_{\varepsilon} / 8$, we know from Proposition 11 that

$$
\tau^{2} \cosh (2 \sigma)+\left|\partial_{s} \tau^{2} \cosh (2 \sigma)\right| \leq \varepsilon^{1 / 2} .
$$


Therefore

$$
\varepsilon^{-1 / 2} e^{(\mu-2) s}\left\|\tau^{2} \cosh (2 \sigma)\right\|_{0, \alpha,[s, s+1]} \leq c \varepsilon^{(\mu-2) S_{\varepsilon} / 8}=c \varepsilon^{1} / 2 \varepsilon^{-\mu / 4} .
$$

Next, when $3 S_{\varepsilon} / 8 \leq s \leq S_{\varepsilon} / 2$, we know, still from Proposition 11, that we may estimate

$$
\tau^{2} \cosh (2 \sigma)+\left|\partial_{s}\left(\tau^{2} \cosh (2 \sigma)\right)\right| \leq c \varepsilon^{2} e^{2 s}
$$

hence

$$
\varepsilon^{-1 / 2} e^{(\mu-2) s}\left\|\tau^{2} \cosh (2 \sigma)\right\|_{0, \alpha,[s, s+1]} \leq c \varepsilon^{-1 / 2+2-\mu}=c \varepsilon^{(6-3 \mu) / 4} \varepsilon^{-\mu / 4} .
$$

Finally, for $S_{\varepsilon} / 2 \leq s$ we use the fact that $\tau^{2} \cosh (2 \sigma) \leq 2$, and proceed as before. This proves the Corollary.

\section{CMC surfaces near to a half unduloid.}

In this section we construct by perturbation methods the full space of CMC surfaces near to a fixed (half) unduloid $\mathcal{D}$ of necksize $\varepsilon$, as usual controlling the behaviour as $\varepsilon \rightarrow 0$. Assume that $\mathcal{D}$ has the parametrization

$$
\mathbf{x}(s, \theta)=\left(\tau e^{\sigma} \cos \theta, \tau e^{\sigma} \sin \theta, k\right),
$$

where $\tau \in(0,1), \sigma$ and $k$ are as in Proposition 2. The unit normal at $\mathbf{x}(s, \theta)$ is defined to be

$$
\nu(s, \theta)=\left(-\tau \cosh \sigma \cos \theta,-\tau \cosh \sigma \sin \theta, \sigma_{s}\right) .
$$

Therefore, surfaces which may be written as normal graphs over $\mathcal{D}$ admit the parametrization

$$
\mathbf{x}_{w}=\mathbf{x}+w \nu
$$

for some sufficiently small function $w$ on $\mathcal{D}$. We denote by $\mathcal{D}_{w}$ the surface obtained in this way. The components of its metric tensor are

$$
E_{w}=\tau^{2}\left(e^{\sigma}-\sinh \sigma w\right)^{2}+w_{s}^{2}, \quad F_{w}=w_{s} w_{\theta},
$$

and

$$
G_{w}=\tau^{2}\left(e^{\sigma}-\cosh \sigma w\right)^{2}+w_{\theta}^{2}
$$


The components of the second fundamental form are considerably less simple. In computing the following, we use that we always have the bounds $\tau e^{\sigma} \leq 2, \tau^{2} \sinh ^{2} \sigma \leq 1$ and $\sigma_{s}^{2} \leq 1$. After substantial work, we find that

$$
\sqrt{E_{w} G_{w}-F_{w}^{2}} L_{w}=\tau^{3} e^{3 \sigma}\left(\tau \sinh \sigma+P_{1}\left(\frac{w}{\tau e^{\sigma}}, \frac{\nabla w}{\tau e^{\sigma}}, \frac{\nabla^{2} w}{\tau e^{\sigma}}\right)\right)
$$

where $P_{1}$ is some polynomial (of degree at most 3 ) without any constant term, the coefficients of which are functions of $s$ and such that they and their derivatives are bounded uniformly in $s$ and $\varepsilon$. In a similar manner we derive that

$$
\sqrt{E_{w} G_{w}-F_{w}^{2}} M_{w}=\tau^{3} e^{3 \sigma} P_{2}\left(\frac{w}{\tau e^{\sigma}}, \frac{\nabla w}{\tau e^{\sigma}}, \frac{\nabla^{2} w}{\tau e^{\sigma}}\right)
$$

and

$$
\sqrt{E_{w} G_{w}-F_{w}^{2}} N_{w}=\tau^{3} e^{3 \sigma}\left(\tau \cosh \sigma+P_{3}\left(\frac{w}{\tau e^{\sigma}}, \frac{\nabla w}{\tau e^{\sigma}}, \frac{\nabla^{2} w}{\tau e^{\sigma}}\right)\right),
$$

where $P_{2}$ and $P_{3}$ have the same properties as $P_{1}$.

The equation that $\mathcal{D}_{w}$ has mean curvature 1 is

$$
L_{w} G_{w}-2 M_{w} F_{w}+N_{w} E_{w}-\left(E_{w} G_{w}-F_{w}^{2}\right)=0 .
$$

This is a rather complicated nonlinear elliptic equation for $w$ which we shall not write out in full. Notice that it is satisfied when $w=0$. Using the previous formula for the coefficients of the first and second fundamental forms, we find that its Taylor expansion about $w=0$ is

$$
L_{\varepsilon} w=\tau e^{\sigma} Q\left(\frac{w}{\tau e^{\sigma}}, \frac{\nabla w}{\tau e^{\sigma}}, \frac{\nabla^{2} w}{\tau e^{\sigma}}\right)
$$

where

$$
L_{\varepsilon} w=\partial_{s s}^{2} w+\partial_{\theta \theta}^{2} w+\tau^{2} \cosh (2 \sigma) w,
$$

and $Q$ is again a polynomial (now of higher order) without any constant or linear terms, the coefficients of which have partial derivatives bounded uniformly in $s$ and $\varepsilon$. We also write, for brevity,

$$
\mathcal{Q}(w):=\tau e^{\sigma} Q\left(\frac{w}{\tau e^{\sigma}}, \frac{\nabla w}{\tau e^{\sigma}}, \frac{\nabla^{2} w}{\tau e^{\sigma}}\right) .
$$


Given $\phi^{\prime \prime} \in \Pi^{\prime \prime}\left(\mathcal{C}^{2, \alpha}\left(S^{1}\right)\right)$, we would like to solve the boundary value problem

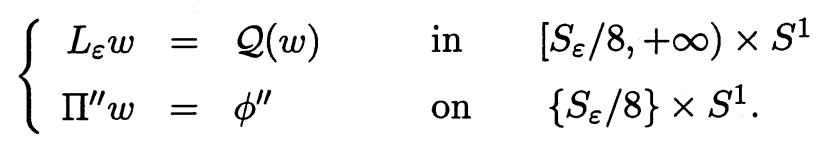

Let $w_{\varepsilon}$ be the unique solution in $\mathcal{C}_{-\mu}^{2, \alpha}\left(\left[S_{\varepsilon} / 8,+\infty\right) \times S^{1}\right), 1<\mu<2$, of

$$
\left\{\begin{array}{lll}
L_{\varepsilon} w_{\varepsilon}=0 & \text { in } & {\left[S_{\varepsilon} / 8,+\infty\right) \times S^{1}} \\
\Pi^{\prime \prime} w_{\varepsilon}=\phi^{\prime \prime} & \text { on } & \left\{S_{\varepsilon} / 8\right\} \times S^{1},
\end{array}\right.
$$

which is given by Proposition 21. Setting $w=w_{\varepsilon}+v$, then we would like to find $v \in \mathcal{C}_{-\mu}^{2, \alpha}\left(\left[S_{\varepsilon} / 8,+\infty\right) \times S^{1}\right)$ such that

$$
\left\{\begin{array}{llll}
L_{\varepsilon} v=\mathcal{Q}\left(w_{\varepsilon}+v\right) & \text { in } & & {\left[S_{\varepsilon} / 8,+\infty\right) \times S^{1}} \\
\Pi^{\prime \prime} v=0 & \text { on } & & \left\{S_{\varepsilon} / 8\right\} \times S^{1} .
\end{array}\right.
$$

Notice that, it is sufficient to find a fixed point of the mapping

$$
\mathcal{K}(v)=G_{\varepsilon} Q\left(w_{\varepsilon}+v\right)
$$

at least when $\varepsilon$ is sufficiently small.

Proposition 22. There exist constants $c_{0}, c_{1}>0$ such that if $\left\|\phi^{\prime \prime}\right\|_{2, \alpha} \leq$ $c_{0} \varepsilon^{3 / 4}$, then

$$
\left\|G_{\varepsilon}\left(\mathcal{Q}\left(w_{\varepsilon}\right)\right)\right\|_{2, \alpha,-\mu} \leq \frac{c_{1}}{2} \varepsilon^{-(\mu+3) / 4}\left\|\phi^{\prime \prime}\right\|_{2, \alpha}^{2}
$$

and

$$
\left\|G_{\varepsilon}\left(\mathcal{Q}\left(w_{\varepsilon}+v_{2}\right)-\mathcal{Q}\left(w_{\varepsilon}+v_{1}\right)\right)\right\|_{2, \alpha,-\mu} \leq 1 / 2\left\|v_{2}-v_{1}\right\|_{2, \alpha,-\mu},
$$

for all $v_{1}, v_{2}$ in $B_{c_{1}}:=\left\{v:\|v\|_{2, \alpha,-\mu} \leq c_{1} \varepsilon^{-(\mu+3) / 4}\left\|\phi^{\prime \prime}\right\|_{2, \alpha}^{2}\right\}$. Thus, $\mathcal{K}$ is a contraction mapping on the ball $B_{c_{1}}$ into itself. Consequently, $\mathcal{K}$ has a unique fixed point $v$ in this ball.

Proof. We shall use that

$$
\left\|w_{\varepsilon}\right\|_{2, \alpha,[s, s+1]} \leq c \varepsilon^{-\mu / 4}\left\|\phi^{\prime \prime}\right\|_{2, \alpha} e^{-\mu s} .
$$


First consider $s$ in the range $\left[S_{\varepsilon} / 8,7 S_{\varepsilon} / 8\right]$. Here, from Proposition 11, we get

$$
\tau e^{\sigma} \geq c \varepsilon^{3 / 4}
$$

Together with the fact that all derivatives of $\sigma$ are bounded, this gives

$$
e^{\mu s}\left\|\mathcal{Q}\left(w_{\varepsilon}\right)\right\|_{0, \alpha,[s, s+1]} \leq c \varepsilon^{-(\mu+3) / 4}\left\|\phi^{\prime \prime}\right\|_{2, \alpha}^{2} .
$$

We chose $c_{1}$ to be equal to twice the constant which appears in this last estimate.

Now

$$
\|v\|_{2, \alpha,[s, s+1]} \leq c_{1} \varepsilon^{-(\mu+3) / 4}\left\|\phi^{\prime \prime}\right\|_{2, \alpha}^{2} e^{-\mu s},
$$

for $v \in B_{c_{1}}$ and we see that

$$
\left.e^{\mu s} \| \mathcal{Q}\left(w_{\varepsilon}+v_{1}\right)-\mathcal{Q}\left(w_{\varepsilon}+v_{2}\right)\right) \|_{0, \alpha,[s, s+1]}
$$

can be estimated by the sum of products of $e^{\mu s}\left\|v_{1}-v_{2}\right\|_{2, \alpha,[s, s+1]}$ with various terms of the form

$$
\left\|\left(w_{\varepsilon}+v_{1}\right)^{j}\left(w_{\varepsilon}+v_{2}\right)^{j^{\prime}}\left(\tau e^{\sigma}\right)^{-j-j^{\prime}}\right\|_{0, \alpha,[s, s+1]}, \quad \text { where } j+j^{\prime} \geq 1 .
$$

Each of these can be bounded by $c\left(c_{0} \varepsilon^{3 / 4}\right)^{j+j^{\prime}} \varepsilon^{-(3 / 4)(i+j)} e^{\left(j+j^{\prime}\right) \mu\left(S_{\varepsilon} / 8-s\right)} \leq$ $c c_{0}^{j+j^{\prime}}$, and so can be made as small as desired provided $c_{0}$ is chosen small enough.

For $s \geq 7 S_{\varepsilon} / 8$, we will simply use the fact that

$$
\tau e^{\sigma} \geq \tau e^{\sigma(0)}=\varepsilon .
$$

Arguing as before, we get first that

$$
e^{\mu s}\left\|\mathcal{Q}\left(w_{\varepsilon}\right)\right\|_{0, \alpha,[s, s+1]} \leq c \varepsilon^{(5 \mu-1) / 4} \varepsilon^{-(\mu+3) / 4}\left\|\phi^{\prime \prime}\right\|_{2, \alpha}^{2} .
$$

Furthermore

$$
\begin{aligned}
\left.e^{\mu s} \| \mathcal{Q}\left(w_{\varepsilon}+v_{1}\right)-\mathcal{Q}\left(w_{\varepsilon}+v_{2}\right)\right) \|_{0, \alpha,[s, s+1]} & \\
& \leq c c_{0} \varepsilon^{-1 / 4} e^{\mu\left(S_{\varepsilon} / 8-s\right)}\left\|v_{2}-v_{1}\right\|_{2, \alpha,[s, s+1]} \\
& \leq c c_{0} \varepsilon^{(6 \mu-1) / 4}\left\|v_{2}-v_{1}\right\|_{2, \alpha,-\mu},
\end{aligned}
$$

and again the coefficient can be made as small as desired when $\varepsilon$ is chosen small enough. 
Putting the estimates in these two domains together, and using that $G_{\varepsilon}$ is bounded, we have now checked all the conditions necessary to ensure that $\mathcal{K}$ is a contraction mapping. Therefore there is a unique element $v \in B_{c_{1}}$ such that $\mathcal{K}(v)=v$, and the proof is complete.

Examining this proof more carefully, we also obtain the

Corollary 5. There exists a constant $c_{0}>0$ and an $\varepsilon_{0}>0$ such that, for all $\varepsilon \in\left(0, \varepsilon_{0}\right)$ and for any $\phi^{\prime \prime} \in \Pi^{\prime \prime}\left(\mathcal{C}^{2, \alpha}\left(S^{1}\right)\right)$ with $\left\|\phi^{\prime \prime}\right\|_{2, \alpha} \leq c_{0} \varepsilon^{3 / 4}$, the problem (5.4) has a unique solution $w$. The mapping

$$
\Pi^{\prime \prime}\left(\mathcal{C}^{2, \alpha}\left(S^{1}\right)\right) \ni \phi^{\prime \prime} \longrightarrow w \in \mathcal{C}_{-\mu}^{2, \alpha}\left(\left[S_{\varepsilon} / 8, \infty\right) \times S^{1}\right),
$$

is continuous and the solution $w$ satisfies the estimates

$$
\|w\|_{2, \alpha,-\mu} \leq c \varepsilon^{-\mu / 4}\left(\left\|\phi^{\prime \prime}\right\|_{2, \alpha}+\varepsilon^{-3 / 4}\left\|\phi^{\prime \prime}\right\|_{2, \alpha}^{2}\right),
$$

and

$$
\left\|\left(w-\Pi^{\prime \prime} w\right)\left(S_{\varepsilon} / 8, \cdot\right)\right\|_{2, \alpha}+\left\|\partial_{s}\left(w-\Pi^{\prime \prime} w\right)\left(S_{\varepsilon} / 8, \cdot\right)\right\|_{1, \alpha} \leq c \varepsilon^{-3 / 4}\left\|\phi^{\prime \prime}\right\|_{2, \alpha}^{2} .
$$

Finally, if $w_{0}=P_{0}\left(\phi^{\prime \prime}\right) \in \mathcal{C}_{-\mu}^{2, \alpha}\left(\left[S_{\varepsilon} / 8,+\infty\right) \times S^{1}\right)$ as in Corollary 4 , then

$$
\left\|w-w_{0}\right\|_{2, \alpha,-\mu} \leq c \varepsilon^{-\mu / 4}\left(\left(\varepsilon^{1 / 2}+\varepsilon^{(6-3 \mu) / 4}\right)\left\|\phi^{\prime \prime}\right\|_{2, \alpha}+\varepsilon^{-3 / 4}\left\|\phi^{\prime \prime}\right\|_{2, \alpha}^{2}\right) .
$$

Proof. The solution $w$ is a sum $w_{\varepsilon}+v$ and we already know that $\left\|w_{\varepsilon}\right\|_{2, \alpha,-\mu} \leq$ $c\left\|\phi^{\prime \prime}\right\|_{2, \alpha}$. For fixed $\phi^{\prime \prime}$, the map $\mathcal{K}$ is a contraction on the balls of radius a constant times $\varepsilon^{-(\mu+3) / 4}\left\|\phi^{\prime \prime}\right\|_{2, \alpha}^{2}$, and so the norm of $v$ is at most this large. And this gives (5.8). The second estimate (5.9) follows by evaluting at $s=S_{\varepsilon} / 8$. Finally, for (5.10), we write

$$
\left\|w-w_{0}\right\|_{2, \alpha,-\mu} \leq\left\|w_{\varepsilon}-w_{0}\right\|_{2, \alpha,-\mu}+\|v\|_{2, \alpha,-\mu},
$$

and use Corollary 4.

\section{CMC surfaces near to a half nodoid.}

We now want to obtain the results which are valid for unduloids in the case of nodoids. More precisely, we want to construct by perturbation methods the 
full space of CMC surfaces near to a fixed nodoid $\mathcal{D}$ of necksize $\varepsilon$, as usual controlling the behaviour as $\varepsilon \rightarrow 0$. Assume that $\mathcal{D}$ has the parametrization

$$
\mathbf{x}(s, \theta)=\left(\tau e^{\bar{\sigma}} \cos \theta, \tau e^{\bar{\sigma}} \sin \theta, \bar{k}\right),
$$

where we retrict our attention to $\tau \in(0,1), \bar{\sigma}$ and $\bar{k}$ are as in Proposition 4. Here it is important to notice that the normal at $\mathbf{x}(s, \theta)$ is defined to be

$$
\nu(s, \theta)=\left(-\tau \sinh \bar{\sigma} \cos \theta,-\tau \sinh \bar{\sigma} \sin \theta, \bar{\sigma}_{s}\right) .
$$

Furthermore, the linearized mean curvature operator is

$$
\overline{\mathcal{L}}_{\varepsilon}=\frac{1}{\tau^{2} e^{2 \bar{\sigma}}}\left(\partial_{s s}^{2}+\partial_{\theta \theta}^{2}+\tau^{2} \cosh (2 \bar{\sigma})\right)
$$

Denote by $\bar{\gamma}_{j}$ the indicial roots of $\overline{\mathcal{L}}_{\varepsilon}$. Proposition 20, which is a key point in the analysis of the mappings properties of $\overline{\mathcal{L}}_{\varepsilon}$, holds with minor modifications in the proof.

The statement and proof of Proposition 21 as well as all the results of $\S 5$ remain the same up to obvious notational modifications.

\section{7. $k$-noids.}

The second type of component in our construction of CMC surfaces are a somewhat restricted class of minimal surfaces of finite total curvature with $k$ ends, or as we shall call them, $k$-noids. In this brief section we discuss some of the global and asymptotic aspects of the geometry and topology of $k$-noids, and in the next, discuss Jacobi operators on these surfaces and their compact truncations.

It is well-known that any $k$-noid $\Sigma$ has finite topology, and in fact is conformally equivalent to the complement of a finite number of points in a compact Riemann surface $\bar{\Sigma}$, i.e. $\Sigma=\bar{\Sigma} \backslash\left\{p_{1}, \ldots, p_{k}\right\}$. As in the introduction, we denote the space of $k$-noids of genus $g$ by $\mathcal{H}_{g, k}$. When $g>0$, $\mathcal{H}_{g, 1}$ and $\mathcal{H}_{g, 2}$ are empty, while $\mathcal{H}_{0,1}$ and $\mathcal{H}_{0,2}$ contain only the plane and catenoid, respectively. The standard catenoid $\mathcal{C}_{1}$ is a surface of revolution, given in cylindrical coordinates by the parametrization

$$
\mathbf{x}(s, \theta)=(\cosh s \cos \theta, \cosh s \sin \theta, s) .
$$

This is a conformal parametrization, and the unit normal, metric tensor and second fundamental forms are given by

$$
\begin{aligned}
\nu(s, \theta) & =\frac{1}{\cosh s}(-\cos \theta,-\sin \theta, \sinh s), \\
g & =\cosh ^{2} s\left(d s^{2}+d \theta^{2}\right), \quad A=-d s^{2}+d \theta^{2} .
\end{aligned}
$$


In particular, the mean curvature vanishes, and the catenoid is minimal.

We shall be discussing the space of moduli of $k$-noids. Just as with CMC surfaces, it is possible to determine the moduli space explicitly in the simplest case, when $k=2$. In fact, the only complete minimal surfaces in $\mathbb{R}^{3}$ with two ends are images of the standard catenoid $\mathcal{C}_{1}$ by rigid motions and homotheties. While we shall frequently not distinguish between $\mathcal{C}_{1}$ and its translates or rotations, it will be important to keep track of the homothety factor. The dilation of $\mathcal{C}_{1}$ by the factor $a$ will be denoted $\mathcal{C}_{a}$, and has the parametrization

$$
\mathbf{x}^{(a)}(s, \theta)=(a \cosh s \cos \theta, a \cosh s \sin \theta, a s) .
$$

Thus any element of $\mathcal{H}_{0,2}$ is given as a rigid motion of some $\mathcal{C}_{a}$. The metric tensor and second fundamental forms for this parametrization are

$$
g_{a}=a^{2} \cosh ^{2} s\left(d s^{2}+d \theta^{2}\right), \quad A_{a}=a\left(-d s^{2}+d \theta^{2}\right) .
$$

The plane and catenoid provide the asymptotic models for the ends of any $k$-noid: the basic structure theorem for $k$-noids states that an end of any $k$-noid may be written as a normal graph of a decaying function over an end of some suitably translated, rotated plane or dilated catenoid. The corresponding ends will then be referred to as planar or catenoidal. Only $k$-noids with all ends catenoidal will be used in our construction; henceforth this will always be assumed.

Using this asymptotics theorem, we may assign a dilation, or weight, parameter $a_{\ell}$ to each end $E_{\ell}$ of $\Sigma \in \mathcal{H}_{g, k}, \ell=1, \ldots, k$, signifying that that end is the normal graph over (some translated and rotated copy of) $\mathcal{C}_{a_{\ell}}$. This is analogous to the necksize parameters of the ends of CMC surfaces. This defines, at least in neighbourhoods of the moduli space where some ordering of the ends is fixed, a map $\mathcal{H}_{g, k} \rightarrow \mathbb{R}^{k}$.

Fix $\Sigma \in \mathcal{H}_{g, k}$. We describe the parametrization of the ends more carefully. Assume that $\Sigma$ has been rotated and translated so that the the end $E_{\ell}$ is asymptotic to the model $\mathcal{C}_{a_{\ell}}$. By definition, there is a function $w$, defined on $\mathcal{C}_{a_{\ell}} \cap\left\{s \geq s_{\ell}\right\}$, such that $E_{\ell}$ is parametrized by

$$
\mathbf{x}_{w}:=\mathbf{x}_{a_{j}}+w \nu, \quad s \geq s_{\ell} .
$$

This gives a canonical cylindrical coordinate system $(s, \theta)$ on $E_{\ell}$, which we will always use. The function $w$ is assumed a priori only to decay, but in fact admits an asymptotic expansion

$$
w(s, \theta) \sim \sum_{|j|>1} a_{j} \chi_{j}(\theta) e^{-j s}, \quad \text { as } \quad s \rightarrow \infty .
$$




\section{The Jacobi operator on $k$-noids.}

Continuing our treatment of analysis on $k$-noids paralleling that on Delaunay surfaces, we now consider the Jacobi operator $L=L_{\Sigma}$, which is the linearization of (half) the mean curvature operator $\mathcal{M}$ over $\Sigma$. This is

$$
L_{\Sigma}=\Delta_{\Sigma}+\left|A_{\Sigma}\right|^{2}
$$

where the term of order zero is the squared norm of the second fundamental form of $\Sigma$.

\subsection{Mapping properties of $L$ and Jacobi fields.}

Just as for Delaunay surfaces, we require detailed knowledge of the mapping properties of $L$, first over all of $\Sigma$, then in a later section for the Dirichlet problem on certain (deformations of) compact truncations $\Sigma_{\varepsilon}$ of $\Sigma$, and finally uniformly as $\varepsilon \rightarrow 0$.

The analysis for $L$ over the complete surface $\Sigma$ is based on the fact that the ends have good asymptotic models. In fact, using the canonical cylindrical coordinates on each end $E_{\ell}$, we see that the Jacobi operator for the model catenoid is

$$
L_{a_{\ell}}=a_{\ell}^{-2} \cosh ^{-2} s\left(\partial_{s s}^{2}+\partial_{\theta \theta}^{2}+\frac{2}{\cosh ^{2} s}\right),
$$

and so the true Jacobi operator is equal, as $s \rightarrow \infty$ in $E_{\ell}$, to the sum of this model operator and a correction term, which is a second order operator each coefficient of which decays at least like $e^{-5 s}$.

We let $L$ act on the weighted Hölder spaces $\mathcal{C}_{\mu}^{r, \alpha}(\Sigma)$, where $\phi$ is in this space if it is locally in $\mathcal{C}^{r, \alpha}(\Sigma)$ and on each end may be written as $e^{\mu s} \psi$ where $\psi \in \mathcal{C}^{r, \alpha}\left(\mathbb{R}_{s}^{+} \times S_{\theta}^{1}\right)$. The basic mapping properties for $L$ are summarized in the

Proposition 23. The operator

$$
L: \mathcal{C}_{\mu}^{2, \alpha}(\Sigma) \longrightarrow \mathcal{C}_{\mu-2}^{0, \alpha}(\Sigma)
$$

is Fredholm provided $\mu \notin \mathbb{Z}$. In addition, $L$ is surjective on $\mathcal{C}_{\mu}^{2, \alpha}(\Sigma)$ if and only if it is injective on $\mathcal{C}_{-\mu}^{2, \alpha}(\Sigma)$.

The drop of two in the weight parameter comes from the factor $\left(\cosh \left(a_{\ell} s\right)\right)^{-2}$ in the expression for $L$ on $E_{\ell}$. This sort of result is fairly 
standard by now; it may be proved by constructing local parametrices, or solution operators, for the model operators on each of the ends $E_{\ell}$ using explicit ODE techniques on each of the cross-sectional eigenspaces, joining these to a parametrix for the interior compact region, and finally using standard perturbation techniques and Fredholm theory.

This Proposition leads naturally to the issue of determining the values of the weight parameters $\mu$ for which $L$ is surjective or injective. Although for a given $k$-noid $\Sigma$ this may be quite difficult to determine, the following condition is essential for the moduli space theory:

Definition 2. A $k$-noid $\Sigma$ is called nondegenerate if its Jacobi operator $L$ is surjective on $\mathcal{C}_{\mu}^{2, \alpha}(\Sigma)$ whenever $\mu>1, \mu \neq 2,3, \ldots$, or equivalently, whenever there are no anomalous decaying Jacobi fields and so $L$ is injective on $\mathcal{C}_{-\mu}^{2, \alpha}(\Sigma)$ for $\mu>1$.

We cannot preclude the existence of Jacobi fields in $\mathcal{C}_{\mu}^{2, \alpha}(\Sigma)$ for $|\mu| \leq 1$, and in fact these always exist, at least locally on each end, for geometric reasons. They may be exhibited explicitly on the catenoid: just as for the Delaunay surfaces, solutions of $L w=0$ corresponding to the eigenvalues of the cross-sectional Laplacian with $|j| \leq 1$ arise from translations, rotations and dilations (which substitute for changes in Delaunay parameter):

Proposition 24. The Jacobi fields

$$
\Psi^{0,+}=\tanh s, \quad \Psi^{0,-}=s \tanh s-1,
$$

correspond to vertical translation along the axis of the catenoid, and change of the dilation parameter a, respectively. The Jacobi fields

$$
\Psi^{1,+}=\psi^{+} \sin \theta, \quad \Psi^{-1,+}=\psi^{+} \sin \theta,
$$

correspond to horizontal translations in the $x_{1}$ and $x_{2}$ directions, while

$$
\Psi^{1,-}=\psi^{-} \cos \theta, \quad \Psi^{-1,-}=\psi^{-} \sin \theta,
$$

correspond to rotations about the $x_{2}$-axis and $x_{1}$-axis, respectively. Here

$$
\psi^{+}=\frac{1}{\cosh s} \quad \psi^{-}=\frac{s}{\cosh s}+\sinh s .
$$

Proof. As with the analogous statement in the Delaunay case, these Jacobi fields may be computed by finding the parametrizations of the one-parameter 
family of minimal surfaces in each case and differentiating to get the deformation vector field, the inner product of which with the unit normal of $\mathcal{C}_{a}$ yields the appropriate expression. We leave the details to the reader.

Jacobi fields asymptotic to these exist on the ends of any $k$-noid. Let $\tilde{\Sigma}$ denote some fixed truncation of the $k$-noid $\Sigma$, and let $E_{1}, \ldots, E_{k}$ denote the components of $\Sigma \backslash \tilde{\Sigma}$. These are in one to one correspondence with the ends of $\Sigma$, and are minimal surfaces with boundary.

Proposition 25. On each end $E_{\ell}$ of $\Sigma$, there exists a six-dimensional space of functions $\Psi_{\ell}^{j, \pm}, j=0, \pm 1$, such that each $L \Psi_{\ell}^{j, \pm}=0$, and which are asymptotic to the corresponding model Jacobi fields $\Psi^{j, \pm}$ for the catenoid $\mathcal{C}_{a_{\ell}}$ modelling $E_{\ell}$ in the sense that

$$
\begin{aligned}
& \left|\Psi_{\ell}^{j,+}-\Psi^{j,+}\right| \leq C e^{-(j+3) s} \\
& \left|\Psi_{\ell}^{j,-}-\Psi^{j,-}\right| \leq C s e^{(j-3) s} .
\end{aligned}
$$

Proof. These new Jacobi fields are produced by the same geometric process, namely forming the families of minimal surfaces with boundary, $E_{\ell}(\eta)$, by translating, rotating or dilating $E_{\ell}$, and then differentiating with respect to the parameter $\eta$ at $\eta=0$, and taking the inner product of the resulting vector field along $E_{\ell}$ with the unit normal. The statement about asymptotics is obtained from the fact that $E_{\ell}$ is a normal graph over $\mathcal{C}_{a_{\ell}}$ of a function $\phi_{\ell}$ which decays like $e^{-2 s}$.

\subsection{Moduli space theory.}

Following these preliminaries, we now briefly sketch the moduli space theory for $k$-noids. This was developed by Perez and Ros [17] at around the same time that the very similar moduli space theory was set down for solutions of the singular Yamabe problem and CMC surfaces in [13] and [9], using slightly different (but equivalent) methods. The parallels between the three problems are discussed carefully in [12]. We state results here following these latter three papers, though emphasize that the results are originally due to Perez and Ros. For $\Sigma \in \mathcal{H}_{g, k}$, define the $6 k$-dimensional space

$$
W=\oplus_{\ell=1}^{k} W_{\ell}, \quad \text { where } \quad W_{\ell}=\left\{\eta_{\ell} \Psi_{\ell}^{j, \pm}, j=0, \pm 1\right\} .
$$


and where $\eta_{\ell}$ is a cutoff function vanishing on $\tilde{\Sigma}$ and equalling one outside of a slight enlargement of this truncation on the end $E_{\ell}$. At least around non-degenerate $k$-noids, the moduli space theory is based on the implicit function theorem. For this one requires surjectivity of $L$ on some geometrically natural function spaces, but unfortunately the spaces on which $L$ is surjective in Proposition 23 have positive exponential weight, hence are ill-suited for the nonlinear operator. To remedy this one uses the following more refined linear result.

Proposition 26. Suppose $\Sigma$ is nondegenerate. Fix $\mu$ with $\mu \in(1,2)$. Then the mapping

$$
L: \mathcal{C}_{-\mu}^{2, \alpha}(\Sigma) \oplus W \longrightarrow \mathcal{C}_{-\mu-2}^{0, \alpha}(\Sigma),
$$

is surjective. Its nullspace, $B:=B_{\Sigma}$ (which we call the bounded nullspace) is $3 k$-dimensional.

The proof is essentially identical to the one in [13] and [9], although the linear theory here is more elementary than the analysis on asymptotically periodic ends in those papers. The dimension count for $B$ is obtained by a relative index theorem (which is essentially equivalent to the Riemann-Roch theorem).

To make sense of the mean curvature operator $N$ on elements of the domain space in (8.2), we use that elements of $W$ correspond to geometric motions. Thus $N\left(u^{\prime}, \tilde{u}\right)$ calculates the mean curvature of the normal graph of the function $\tilde{u} \in \mathcal{C}_{-\mu}^{2, \alpha}$ over the surface $\Sigma_{u^{\prime}}$ obtained by slightly deforming the ends of $\Sigma$ in the manner prescribed by the components of $u^{\prime} \in W$. (More specifically, one considers an 'exponential map' from a neighbourhood of 0 in $W$ to a space of surfaces deforming $\Sigma$, such that the derivatives of the families of surfaces $\Sigma\left(\lambda u^{\prime}\right)$, for $u^{\prime} \in W$, at $\lambda=0$ equal $u^{\prime}$.) Proposition 26 then states that the differential of this map $N$ is surjective at $(0,0)$ when $\Sigma$ is nondegenerate, and so the first part of the following is a trivial application of the standard implicit function theorem:

Corollary 6. In the neighbourhood of any one of its nondegenerate points, the moduli space $\mathcal{H}_{g, k}$ of $k$-noids of genus $g$ is a real analytic manifold of dimension $3 k$. In the neighbourhood of an arbitrary point, it has the structure of a locally defined (possibly singular) real analytic variety.

Perez and Ros note that the second part of this result follows from the general theory of Weierstrass representations of $k$-noids. It may also be 
proved in a manner more consistent with the first part by the Kuranishi method, as in [9], cf. also [12].

\subsection{Existence of nondegenerate $k$-noids.}

We now briefly survey the results in the literature concerning the existence of nondegenerate $k$-noids; we also state some new existence results which may be deduced from these. This survey is included to show that our construction yields many new examples of CMC surfaces.

Many of the moduli spaces $\mathcal{H}_{g, k}$ are known to be nonempty. The Weierstrass representation yields some infinite families of examples of complete, Alexandrov embedded minimal surfaces of finite total curvature, while Kapouleas' desingularization construction also gives examples when $g$ is quite large relative to $k$. It is quite plausible that all of these moduli spaces, when nonempty, contain nondegenerate elements; unfortunately this has been proved only in special cases. There are two main results in this regard which we quote here. The first, by Cosin and Ros [1] and already stated in the introduction, gives the existence of nondegenerate elements in $\mathcal{H}_{0, k}$ for any $k \geq 2$. Incidentally, all of these surfaces have catenoidal ends. Secondly, the Hoffman-Meeks minimal surfaces give elements of $\mathcal{H}_{g, 3}$ for any $g \geq 1$. Nayatani [15] has shown that these are nondegenerate when $g \leq 37$. Unfortunately, the 'middle' end of these surfaces is planar, but using the nondegeneracy it is possible to find nearby surfaces which are nondegenerate and for which all ends are catenoidal.

These two theorems provide nondegenerate $k$ noids for infinitely many pairs $(g, k)$. Using them in the the connected sum construction of [11] we can construct many more examples. Although the results in that paper are written for CMC surfaces, it is quite easy to check that the constructions there carry over verbatim for nondegenerate minimal surfaces. In particular, nondegeneracy of the resulting surfaces (which turns out to be somewhat delicate) is proved. Yang [21] explicitly addresses the problem of connected sums of complete minimal surfaces, but does not consider the nondegeneracy issue. In any case, we conclude that if $\Sigma_{i}$ are non degenerate elements of $\mathcal{H}_{g_{i}, k_{i}}, i=1, \ldots, r$, then an iterated connected sum of the $\Sigma_{i}$ gives a nondegenerate element of $\mathcal{H}_{g, k}, g=\sum_{i=1}^{r} g_{i}, k=\sum_{i=1}^{r} k_{i}$. In addition, following Yang (as well as recent work of the second author and Fakhi in higher dimensions), it is possible to glue half-catenoids to any nondegenerate surface, and again, the resulting surface may be proved to be nondegenerate. Altogether we obtain 
Proposition 27. Suppose that $\mathcal{H}_{g_{i}, k_{i}}$ contains a nondegenerate element, $i=1, \ldots, r$. Then $\mathcal{H}_{g, k}$ also contains a nondegenerate element provided $g=\sum_{i=1}^{r} g_{i}$ and $k \geq \sum_{i=1}^{4} k_{i}$.

There is a similar result for the moduli spaces $\mathcal{M}_{g, k}$ of CMC surfaces. We shall return to this issue, as well as a number of other interesting new aspects of these moduli spaces, in a forthcoming paper.

\section{Truncated $k$-noids and their deformations.}

In this section we first introduce the compact truncations $\Sigma_{\varepsilon}$ which fill out the $k$-noid $\Sigma$ as $\varepsilon \rightarrow 0$. These are the building blocks occupying the central portion of the surfaces we shall construct. The reason for introducing them is that there are no surfaces of mean curvature one which may be written as normal graphs over all of $\Sigma$, but there are many which are graphs over any one of the $\Sigma_{\varepsilon}$. Next we study a natural boundary problem for the Jacobi operator on these compact surfaces and analyze its behaviour as $\varepsilon$ tends to zero. Finally, we introduce a finite dimensional family of deformations of the Jacobi operator, corresponding to the elements of $W$, and show that the preceding linear analysis carries over to the operators in this family.

\subsection{The Jacobi operator on truncated $k$-noids.}

We start by defining the truncations $\Sigma_{\varepsilon}$. Recall that each end $E_{\ell}$ of $\Sigma$ admits the parametrization

$$
\mathbf{x}_{\ell}(s, \theta)=a_{\ell}\left(\cosh s \cos \theta+O\left(e^{-3 s}\right), \cosh s \sin \theta+O\left(e^{-3 s}\right), s+O\left(e^{-2 s}\right)\right) .
$$

We simply define $\Sigma_{\varepsilon}$ to be the union of the compact piece $K$ of $\Sigma$ and the portion of each of the ends up to $s=S_{\varepsilon} / 8$ (which is of the order $-\frac{1}{4} \log \varepsilon$ ).

Preliminary to the nonlinear analysis, in the next section, of the family of surfaces of constant mean curvature one which are normal graphs over the $\Sigma_{\varepsilon}$, we shall require information about a certain inhomogeneous boundary problem for the Jacobi operator $L$ on $\Sigma_{\varepsilon}$, in particular its solvability and the uniformity of this solution with respect to $\varepsilon$.

We will identify the space $\mathcal{C}^{2, \alpha}\left(\partial \Sigma_{\varepsilon}\right)$ with $\left[\mathcal{C}^{2, \alpha}\left(S^{1}\right)\right]^{k}$ and, for later use we also define

$$
\mathcal{C}^{2, \alpha}\left(\partial \Sigma_{\varepsilon}\right)^{\prime \prime}=\left\{w \in \mathcal{C}^{2, \alpha}\left(\partial \Sigma_{\varepsilon}\right):\left.w\right|_{E_{\ell}}:=w_{\ell}(\theta) \in \operatorname{Span}\left\{\chi_{j}(\theta)\right\}_{|j| \geq 2}\right\}
$$


We now set up the boundary problem for the Jacobi operator on the surfaces $\Sigma_{\varepsilon}$. That the mapping

$$
L:\left\{u \in \mathcal{C}^{2, \alpha}\left(\Sigma_{\varepsilon}\right):\left.u\right|_{\partial \Sigma_{\varepsilon}}=0\right\} \longrightarrow \mathcal{C}^{0, \alpha}\left(\Sigma_{\varepsilon}\right),
$$

is surjective when is $\varepsilon$ is sufficiently small is fairly easy to establish. Unfortunately, the norm of the inverse is not uniformly bounded as $\varepsilon \rightarrow 0$. This happens for a good reason: the range of the inverse may be too close to the restriction of the bounded nullspace $B$ to $\Sigma_{\varepsilon}$. Therefore we impose a boundary condition, the corresponding solution operator for which does have the uniformity we need later.

Finally, for $f \in \mathcal{C}_{\mu-2}^{0, \alpha}(\Sigma)$ and $\phi^{\prime \prime} \in \mathcal{C}^{2, \alpha}\left(\partial \Sigma_{\varepsilon}\right)^{\prime \prime}$, consider the boundary problem

$$
\left\{\begin{array}{rlll}
L u & =\left.f\right|_{\Sigma_{\varepsilon}} & \text { in } & \Sigma_{\varepsilon} \\
\Pi^{\prime \prime}(u) & =\phi^{\prime \prime} & \text { on } & \partial \Sigma_{\varepsilon} .
\end{array}\right.
$$

Proposition 28. Fix $\mu$ with $\mu \in(1,2)$. There exists an $\varepsilon_{0}>0$ and for all $\varepsilon \in\left(0 ? \varepsilon_{0}\right)$ linear maps

$$
\begin{aligned}
\bar{G}_{\varepsilon}: \mathcal{C}_{\mu-2}^{0, \alpha}\left(\Sigma_{\varepsilon}\right) & \longrightarrow \mathcal{C}_{\mu}^{2, \alpha}\left(\Sigma_{\varepsilon}\right) \\
\varepsilon^{\mu / 4} \bar{P}_{\varepsilon}:\left(\mathcal{C}^{2, \alpha}\left(\partial \Sigma_{\varepsilon}\right)\right)^{\prime \prime} & \longrightarrow \mathcal{C}_{\mu}^{2, \alpha}\left(\Sigma_{\varepsilon}\right),
\end{aligned}
$$

which are bounded uniformly for $\varepsilon \in\left(0, \varepsilon_{0}\right)$ such that if $f \in \mathcal{C}_{\mu-2}^{0, \alpha}(\Sigma)$ and $\phi^{\prime \prime} \in \mathcal{C}^{2, \alpha}\left(\partial \Sigma_{\varepsilon}\right)^{\prime \prime}$, then $w=\bar{G}_{\varepsilon}(f)+\bar{P}_{\varepsilon}\left(\phi^{\prime \prime}\right)$ is a solution of (9.2).

Proof. To start, use a bounded extension operator in the $\mathcal{C}^{2, \alpha}\left(\partial \Sigma_{\varepsilon}\right) \rightarrow$ $\mathcal{C}_{\mu}^{2, \alpha}(\Sigma)$ in the usual way to reduce to the case where $\phi^{\prime \prime}=0$.

We are going to show that, for all $\varepsilon$ small enough, there exists

$$
\hat{G}_{\varepsilon}: \mathcal{C}_{\mu-2}^{0, \alpha}\left(\Sigma_{\varepsilon}\right) \longrightarrow \mathcal{C}_{\mu}^{2, \alpha}\left(\Sigma_{\varepsilon}\right),
$$

which is uniformly bounded as $\varepsilon$ tends to 0 and for which

$$
\left\|L \hat{G}_{\varepsilon}(f)-f\right\|_{0, \alpha, \mu-2} \leq c \varepsilon^{\mu / 2}\|f\|_{0, \alpha, \mu-2}
$$

The proposition follows from this claim by perturbation.

First step. Let us choose $s_{m}$ fixed larger than any of the $s_{\ell}$. For each end $E_{\ell}$ of $\Sigma$, we find a solution $\bar{u}_{\ell} \in \mathcal{C}_{\mu}^{2, \alpha}\left(E_{\ell}\right)$ of

$$
\left\{\begin{aligned}
L_{a_{\ell}} \bar{u}_{\ell} & =\left.f\right|_{E_{\ell}} & & \text { in } & & \left(s_{m}, S_{\varepsilon} / 8\right) \times S^{1} \\
\left.\bar{u}_{\ell}\right|_{\partial E_{\ell}} & =0 & & \text { on } & & \left\{s_{m}\right\} \times S^{1} \\
\left.\Pi^{\prime \prime} \bar{u}_{\ell}\right|_{\partial E_{\ell}} & =0 & & \text { on } & & \left\{S_{\varepsilon} / 8\right\} \times S^{1} .
\end{aligned}\right.
$$


This solution is not unique, but we can proceed as in the proof of Proposition 21 and first project the operator $L_{a_{\ell}}$ over the eigenspaces $\chi_{j}$ and then chose the (unique) right inverse for which $\Pi^{\prime}\left(\partial_{s} \bar{u}_{\ell}\right)=0$ on $\left\{s_{m}\right\} \times S^{1}$. Let us denote by $G_{\ell}$ this right inverse. Following the proof of Proposition 21, it is easy to see that the norm of $G_{\ell}$ is bounded independently of $\varepsilon$ and also of $s_{m} \geq \sup _{\ell} s_{\ell}$.

Now, using a perturbation argument, we can always assume, increasing $s_{m}$ if necessary, that the result is also true when $L_{a_{\ell}}$ is replaced by $L$ since we have

$$
\left\|\left(L-L_{a_{\ell}}\right) w\right\|_{0, \alpha, \mu-2} \leq c e^{-3 s_{\ell}}\|w\|_{2, \alpha, \mu} .
$$

We denote by $u_{\ell}$ the corresponding solutions. We can patch together the functions $u_{\ell}$ to all $\Sigma_{\varepsilon}$ by defining $v:=\chi \bar{u}_{\ell}$, where $\chi$ is smooth, vanishes for $s \leq s_{m}+1$ and equals one for $s \geq s_{m}+2$ on each $E_{\ell}$. Since $L v=f_{\ell}$ in each end $E_{\ell}$, we have now reduced our problem to the case where on the right hand side of (9.2) the function is supported in a compact set which is independent of $\varepsilon$. We set $g:=f-L v$ and we extend this function by 0 on each end. The norm of this extension is bounded in $\mathcal{C}_{-\mu-2}^{0, \alpha}(\Sigma)$ by a constant times the norm of $f$ in $\mathcal{C}_{\mu}^{0, \alpha}\left(\Sigma_{\varepsilon}\right)$.

Second step. With the help of Proposition 26, we can solve globally in $\Sigma$

$$
L w=g,
$$

where this time $g$ has support away from the ends and $w \in \mathcal{C}_{-\mu}^{2, \alpha} \oplus W$. Near each end $E_{\ell}$ we can decompose

$$
\left.w\right|_{E_{\ell}}=\Pi^{\prime}\left(\left.w\right|_{E_{\ell}}\right)+\Pi^{\prime \prime}\left(\left.w\right|_{E_{\ell}}\right) .
$$

Finally, we modify $w$ near each $\partial E_{\ell}$ by

$$
\tilde{w}=\Pi^{\prime}\left(\left.w\right|_{E_{\ell}}\right)+\tilde{\chi} \Pi^{\prime \prime}\left(\left.w\right|_{E_{\ell}}\right),
$$

where $\tilde{\chi}$ is smooth, vanishes for $s \geq S_{\varepsilon} / 8$ and equals one for $s \leq S_{\varepsilon} / 8-1$. Naturally $L(\tilde{w}+v)=f$ everywhere except near each $\partial E_{\ell}$. Moreover, making use of Proposition 25, it is then a simple exercise to prove that

$$
\|L(\tilde{w}+v)-f\|_{0, \alpha, \mu-2} \leq c e^{-\mu S_{\varepsilon} / 4}\|f\|_{0, \alpha, \mu-2} .
$$

We define $\hat{G}_{\varepsilon}(f):=\tilde{w}+v$. The proof of the claim is complete. 


\subsection{Deformations of $\Sigma_{\varepsilon}$.}

Now we shall take up the task of defining slightly different truncations of the scaled surface $\varepsilon \Sigma$, which we shall call $\tilde{\Sigma}_{\varepsilon, \mathcal{P}}$ (the $\mathcal{P}$ here refers to a parameter set which we shall define below), which will be more convenient later. In the next subsection we shall also consider the Jacobi operators which correspond to writing nearby surfaces as graphs using vector fields which are small deformations of the normal vector field on $\tilde{\Sigma}_{\varepsilon, \mathcal{P}}$.

Fix one end $E_{\ell}$ of $\Sigma$, and recall its parametrization (9.1). The end $\varepsilon E_{\ell}$ can therefore be parametrized as

(9.3) $\mathbf{x}_{\ell}=\varepsilon_{\ell}\left(\cosh s \cos \theta+O\left(e^{-3 s}\right), \cosh s \sin \theta+O\left(e^{-3 s}\right), s+O\left(e^{-2 s}\right)\right)$.

where here and later, we use the notation

$$
\varepsilon_{\ell}:=a_{\ell} \varepsilon
$$

There is a Delaunay surface $\mathcal{D}_{\ell}$, with Delaunay parameter $\varepsilon_{\ell}$, which 'best fits' the model catenoid for $\varepsilon E_{\ell}$ near the region where $s=S_{\varepsilon_{\ell}} / 8$ and whose orientation in this region is compatible with the chosen orientation of $\Sigma$. If $\mathcal{D}_{\ell}$ is an unduloid, it will have the parametrization

$$
\mathbf{x}_{\mathcal{D}_{\ell}}=\left(\tau_{\ell} e^{\sigma_{\ell}} \cos \theta, \tau_{\ell} e^{\sigma_{\ell}} \sin \theta, k_{\ell}\right)
$$

and unit normal

$$
\nu_{\mathcal{D}_{\ell}}=\left(-\tau_{\ell} \cosh \left(\sigma_{\ell}\right) \cos \theta,-\tau_{\ell} \cosh \left(\sigma_{\ell}\right) \sin \theta,\left(\sigma_{\ell}\right)_{s}\right) .
$$

If $\mathcal{D}_{\ell}$ is a nodoid, it will have the parametrization

$$
\mathbf{x}_{\mathcal{D}_{\ell}}=\left(\tau_{\ell} e^{\bar{\sigma}_{\ell}} \cos \theta, \tau_{\ell} e^{\bar{\sigma}_{\ell}} \sin \theta, \bar{k}_{\ell}\right)
$$

and unit normal

$$
\nu_{\mathcal{D}_{\ell}}=\left(-\tau_{\ell} \sinh \left(\bar{\sigma}_{\ell}\right) \cos \theta,-\tau_{\ell} \sinh \left(\bar{\sigma}_{\ell}\right) \sin \theta,\left(\bar{\sigma}_{\ell}\right)_{s}\right) .
$$

Remark 2. For simplicity in the notation and statement of results, we will henceforth assume that $\Sigma \in \mathcal{H}_{g, k}^{s}$. This means that all ends of $\Sigma$ are asymptotic to a catenoid whose orientation (induced by the orientation of $\Sigma$ ) is the same for all ends. We shall also assume that all $\mathcal{D}_{\ell}$ are unduloids. We could equally well choose all $\mathcal{D}_{\ell}$ to be nodoids and this would require only minor changes in the subsequent arguments. When $\Sigma \in \mathcal{H}_{g, k}^{m}$, so that its ends are asymptotic to a catenoid whose orientation (induced by the orientation of $\Sigma$ ) depends on the end, we may choose $\mathcal{D}_{\ell}$ to be a nodoid when the catenoid has positive orientation and an unduloid when it has negative orientation, or vice versa. 
The deformations of $\mathcal{D}_{\ell}$ are parametrized by translations orthogonal to, rotations of and translations along this axis and also changes in the Delaunay parameter. We label these by

$$
\mathcal{P}^{\ell}=\left(t_{1}^{\ell}, t_{2}^{\ell}, r_{1}^{\ell}, r_{2}^{\ell}, d_{\ell}, \delta^{\ell}\right),
$$

respectively. All these parameters lie in some small neighbourhood of zero. (The $r$ 's are identified with some small neighbourhood of the identity in the space of rotations fixing the $x_{3}$ axis; the exact manner is not important, but to be definite, we suppose that the diffeomorphism is given by the exponential map in $\mathrm{SO}_{3}$ orthogonal to the copy of $\mathrm{SO}_{2}$ which is the stabilizer of that axis, followed by the projection to $\mathrm{SO}_{3} / \mathrm{SO}_{2}$.) The full parameter set for all ends of $\varepsilon \Sigma_{\varepsilon}$ is

$$
\mathcal{P}=\left(\mathcal{P}^{1}, \ldots, \mathcal{P}^{k}\right)
$$

We also set

$$
\begin{gathered}
\tilde{t}=\left(t_{1}^{1}, \ldots, t_{2}^{k}\right) \in \mathbb{R}^{2 k}, \quad \tilde{r}=\left(r_{1}^{1}, \ldots, r_{2}^{k}\right) \in \mathbb{R}^{2 k}, \\
\tilde{d}=\left(d^{1}, \ldots, d^{k}\right) \in \mathbb{R}^{k}, \quad \text { and } \quad \tilde{\delta}=\left(\delta^{1}, \ldots, \delta^{k}\right) \in \mathbb{R}^{k},
\end{gathered}
$$

and the rigid motion determined by $\left(t_{1}^{\ell}, t_{2}^{\ell}, r_{1}^{\ell}, r_{2}^{\ell}, d^{\ell}\right)$ will be denoted $\mathcal{R}_{\left(t^{\ell}, r^{\ell}, d^{\ell}\right)}$. The norm on these parameter sets which arises naturally below is given by

$$
\|\mathcal{P}\|=\|(\tilde{t}, \tilde{r}, \tilde{d}, \tilde{\delta})\|:=\varepsilon^{1 / 4}\|\tilde{t}\|+\varepsilon^{3 / 4}\|\tilde{r}\|+\left(\log \frac{1}{\varepsilon}\right)^{-1}\|\tilde{d}\|+\|\tilde{\delta}\| .
$$

The Delaunay surface associated to the set of (small) deformation parameters $\mathcal{P}_{\ell}$ will be denoted $\mathcal{D}_{\mathcal{P} \ell}$, and its induced parametrization and unit normal will be called $\mathbf{x}_{\mathcal{P} \ell}$ and $\nu_{\mathcal{p} \ell}$, respectively. This surface has Delaunay parameter $\varepsilon_{\ell}+\delta^{\ell}$.

We come now to the main point, which is to write a neighbourhood of $\varepsilon\left(E_{\ell} \cap \partial \Sigma_{\varepsilon}\right)$ as a normal graph over each $\mathcal{D}_{\mathcal{P} \ell}$, and to obtain estimates on the graph function.

Proposition 29. Fix $\kappa \in\left(1, \frac{3}{2}\right)$. Then, for all parameter sets $\mathcal{P}$ with $\|\mathcal{P}\| \leq$ $\varepsilon^{\kappa}$, there is a diffeomorphism $\Psi(s, \theta)=\left(s^{\prime}, \theta^{\prime}\right)$ from $\left(-2+S_{\varepsilon_{\ell}} / 8, S_{\varepsilon_{\ell}} / 8\right) \times S^{1}$ onto its image, satisfying

$$
\|\Psi(s, \theta)-(s, \theta)\|=O\left(\varepsilon^{\kappa-1}\right),
$$


and we have

$$
\mathbf{x}_{\ell}(s, \theta)=\mathbf{x}_{\mathcal{P}^{\ell}}\left(s^{\prime}, \theta^{\prime}\right)+\hat{w}_{0}\left(s^{\prime}, \theta^{\prime}\right) \nu_{\mathcal{P}^{\ell}}\left(s^{\prime}, \theta^{\prime}\right)
$$

for all $\varepsilon<\varepsilon_{0}$, where $\varepsilon_{0}$ depends only on $\kappa$. The graph function $\hat{w}_{0}$ here is of the form

$$
\begin{aligned}
\hat{w}_{0}\left(s^{\prime}, \theta^{\prime}\right)= & -\frac{1}{\cosh s^{\prime}}\left(t_{1}^{\ell} \cos \theta^{\prime}+t_{2}^{\ell} \sin \theta^{\prime}\right)-\left(r_{1}^{\ell} \cos \theta^{\prime}+r_{2}^{\ell} \sin \theta^{\prime}\right) \varepsilon_{\ell} \cosh s^{\prime} \\
& +d^{\ell}+\delta^{\ell}\left(s^{\prime}-1\right)+O\left(\varepsilon^{3 / 2}+\varepsilon^{2 \kappa-1}\right) .
\end{aligned}
$$

In other words, we are writing a neighbourhood of $\varepsilon\left(E_{\ell} \cap \partial \Sigma_{\varepsilon}\right)$ as a normal graph over each of the family of nearby model Delaunay surfaces, up to the reparametrization given by the diffeomorphism $\Psi$.

Proof. This follows from a computation similar to the one we have already done in the proof of Proposition 18. Recall that in the range $s \in[-4+$ $\left.S_{\varepsilon} / 8,4+S_{\varepsilon} / 8\right]$, we have the expansions

$$
k=\varepsilon s+O\left(\varepsilon^{3 / 2}\right), \quad \tau e^{\sigma}=\varepsilon \cosh s+O\left(\varepsilon^{5 / 4}\right),
$$

and

$$
\tau \cosh \sigma=\frac{1}{\cosh s}+O\left(\varepsilon^{3 / 4}\right), \quad \partial_{s} \sigma=1+O\left(\varepsilon^{1 / 2}\right),
$$

which follow from (3.25). Here and below $O\left(\varepsilon^{\gamma}\right)$ will denote functions of $(s, \theta)$ all derivatives of which are bounded by constant multiples of $\varepsilon^{\gamma}$.

It will be most convenient to apply the transformation $\mathcal{R}_{\left(t^{\ell}, r^{\ell}, d^{\ell}\right)}^{-1}$ to both sides of (9.5). On the one hand, from (9.1), the parametrization for $\mathcal{R}_{\left(t^{\ell}, r^{\ell}\right)}^{-1}\left(\mathbf{x}_{\ell}(s, \theta)\right)$ is given by

$$
\begin{aligned}
(s, \theta) \longrightarrow & \left(\varepsilon_{\ell} \cosh s \cos \theta-t_{1}^{\ell}+O\left(\varepsilon^{\kappa+1 / 4} \log \varepsilon\right),\right. \\
& \varepsilon_{\ell} \cosh s \sin \theta-t_{2}^{\ell}+O\left(\varepsilon^{\kappa+1 / 4} \log \varepsilon\right), \\
& \left.\left(r_{1}^{\ell} \cos \theta+r_{2}^{\ell} \sin \theta\right) \varepsilon_{\ell} \cosh s+\varepsilon_{\ell} s-d^{\ell}+O\left(\varepsilon^{3 / 2}+\varepsilon^{2 \kappa-3 / 4}\right)\right)
\end{aligned}
$$

for $s$ in this range.

On the other hand, $\mathcal{R}_{\left(t^{\ell}, r^{\ell}, d^{\ell}\right)}^{-1}\left(\mathbf{x}_{\mathcal{P}^{\ell}}\left(s^{\prime}, \theta^{\prime}\right)+\hat{w}_{0}\left(s^{\prime}, \theta^{\prime}\right) \nu_{\mathcal{P}^{\ell}}\left(s^{\prime}, \theta^{\prime}\right)\right)$ is 
parametrized by

$$
\begin{aligned}
& \left(s^{\prime}, \theta^{\prime}\right) \longrightarrow \\
& \left(\left(\left(\varepsilon_{\ell}+\delta^{\ell}\right) \cosh s^{\prime}-\frac{1}{\cosh s^{\prime}} \hat{w}_{0}\left(s^{\prime}, \theta^{\prime}\right)\right) \cos \theta^{\prime}+O\left(\varepsilon^{5 / 4}\right)+O\left(\varepsilon^{3 / 4}\right) \hat{w}_{0}\left(s^{\prime}, \theta^{\prime}\right),\right. \\
& \left(\left(\varepsilon_{\ell}+\delta^{\ell}\right) \cosh s^{\prime}-\frac{1}{\cosh s^{\prime}} \hat{w}_{0}\left(s^{\prime}, \theta^{\prime}\right)\right) \sin \theta^{\prime}+O\left(\varepsilon^{5 / 4}\right)+O\left(\varepsilon^{3 / 4}\right) \hat{w}_{0}\left(s^{\prime}, \theta^{\prime}\right), \\
& \left.\left(\varepsilon_{\ell}+\delta^{\ell}\right) s^{\prime}+\hat{w}_{0}\left(s^{\prime}, \theta^{\prime}\right)+O\left(\varepsilon^{3 / 2}\right)+O\left(\varepsilon^{1 / 2}\right) \hat{w}_{0}\left(s^{\prime}, \theta^{\prime}\right)\right),
\end{aligned}
$$

again for $s^{\prime}$ in this range.

Equating the third coordinates, we already find that $\hat{w}_{0}\left(s^{\prime}, \theta^{\prime}\right)=\varepsilon(s-$ $\left.s^{\prime}\right)+O\left(\varepsilon^{\kappa} \log \varepsilon\right)$. Assuming that $\left|s^{\prime}-s\right|$ is at least bounded, this gives $\hat{w}_{0}\left(s^{\prime}, \theta^{\prime}\right)=O(\varepsilon)$. Similar estimates hold for its derivatives. Now, writing out the equality of the three coordinates in turn gives

$$
\begin{aligned}
& \varepsilon_{\ell} \cosh s \cos \theta=t_{1}^{\ell}+\left(\left(\varepsilon_{\ell}+\delta^{\ell}\right) \cosh s^{\prime}-\frac{1}{\cosh s^{\prime}} \hat{w}_{0}\left(s^{\prime}, \theta^{\prime}\right)\right) \cos \theta^{\prime}+O\left(\varepsilon^{5 / 4}\right) \\
& \varepsilon_{\ell} \cosh s \sin \theta=t_{2}^{\ell}+\left(\left(\varepsilon_{\ell}+\delta^{\ell}\right) \cosh s^{\prime}-\frac{1}{\cosh s^{\prime}} \hat{w}_{0}\left(s^{\prime}, \theta^{\prime}\right)\right) \sin \theta^{\prime}+O\left(\varepsilon^{5 / 4}\right),
\end{aligned}
$$

and

$$
\begin{aligned}
\left(r_{1}^{\ell} \cos \theta+r_{2}^{\ell} \sin \theta\right) \varepsilon_{\ell} \cosh s & +\varepsilon_{\ell} s-d^{\ell} \\
= & \left(\varepsilon_{\ell}+\delta^{\ell}\right) s^{\prime}+\hat{w}_{0}\left(s^{\prime}, \theta^{\prime}\right)+O\left(\varepsilon^{3 / 2}+\varepsilon^{2 \kappa-3 / 4}\right) .
\end{aligned}
$$

Using the preliminary estimate on $\hat{w}_{0}$ together with the first identities, we conclude that

$$
\left|s-s^{\prime}\right| \leq c \varepsilon^{\kappa-1}, \quad\left|\theta^{\prime}-\theta\right| \leq c \varepsilon^{\kappa-1},
$$

and then, reinserting this information back into the third equality, that

$$
\left|\hat{w}_{0}\right| \leq c \varepsilon^{\kappa}|\log \varepsilon|,
$$

along with its derivatives. The third identity gives

$$
\begin{aligned}
\hat{w}_{0}\left(s^{\prime}, \theta^{\prime}\right)= & -\varepsilon_{\ell}\left(s-s^{\prime}\right) \\
& -\left(r_{1}^{\ell} \cos \theta^{\prime}+r_{2}^{\ell} \sin \theta^{\prime}\right) \varepsilon_{\ell} \cosh s^{\prime}+d^{\ell}+\delta^{\ell} s^{\prime}+O\left(\varepsilon^{3 / 2}+\varepsilon^{2 \kappa-1}\right),
\end{aligned}
$$

while from the first two identities we get

$$
\varepsilon_{\ell}\left(\cosh s-\cosh s^{\prime}\right)=t_{1}^{\ell} \cos \theta^{\prime}+t_{2}^{\ell} \sin \theta^{\prime}+\delta^{\ell} \cosh s^{\prime}+O\left(\varepsilon^{5 / 4}+\varepsilon^{2 \kappa-5 / 4}\right) \text {. }
$$


This leads finally to

$$
\begin{aligned}
& \hat{w}_{0}\left(s^{\prime}, \theta^{\prime}\right)=-\frac{1}{\cosh s^{\prime}}\left(t_{1}^{\ell} \cos \theta^{\prime}+t_{2}^{\ell} \sin \theta^{\prime}\right)- \\
& \quad\left(r_{1}^{\ell} \cos \theta^{\prime}+r_{2}^{\ell} \sin \theta^{\prime}\right) \varepsilon_{\ell} \cosh s^{\prime}+d^{\ell}+\delta^{\ell}\left(s^{\prime}-1\right)+O\left(\varepsilon^{3 / 2}+\varepsilon^{2 \kappa-1}\right),
\end{aligned}
$$

which is the desired expansion.

We may now define the deformation $\tilde{\Sigma}_{\varepsilon, \mathcal{P}}$ when the set of deformation parameters $\mathcal{P}$ satisfies $\|\mathcal{P}\| \leq \varepsilon^{\kappa}$. Choose $\varepsilon_{0}$ sufficiently small that $S_{\varepsilon_{\ell}} / 8-$ $2>s_{\ell}$ for each $\ell$ whenever $\varepsilon<\varepsilon_{0}$. Then for any such $\varepsilon$, define $\tilde{\Sigma}_{\varepsilon, \mathcal{P}}$ as the union of the central compact portion of $\varepsilon \Sigma_{\varepsilon}$ and the portion of each end $\varepsilon E_{\ell}$ for $s_{\ell} \leq s \leq-1+S_{\varepsilon_{\ell}} / 8$ and the graph of

$$
\left(s^{\prime}, \theta^{\prime}\right) \longrightarrow \mathbf{x}_{\mathcal{P}^{\ell}}\left(s^{\prime}, \theta^{\prime}\right)+\hat{w}_{0}\left(s^{\prime}, \theta^{\prime}\right) \nu_{\mathcal{P}^{\ell}}\left(s^{\prime}, \theta^{\prime}\right)
$$

for $-2+S_{\varepsilon_{\ell}} / 8 \leq s^{\prime} \leq S_{\varepsilon_{\ell}} / 8$.

Remark 3. These definitions are compatible in the region of overlap, and all we have done is to slightly alter the boundary of $\tilde{\Sigma}_{\varepsilon, \mathcal{P}}$ so that it conforms better to the coordinates $\left(s^{\prime}, \theta^{\prime}\right)$.

\subsection{Deformed Jacobi operators.}

For any small parameter set $\mathcal{P}$, we define on the surface $\tilde{\Sigma}_{\varepsilon, \mathcal{P}}$ a vector field $\tilde{\nu}$ which is the unit normal vector field away from the boundary, and which is a perturbation of this unit normal near to the boundary. More specifically, write $\varepsilon E_{\ell}$ as the graph

$$
(s, \theta) \longrightarrow \mathbf{x}_{\mathcal{P}^{\ell}}+\hat{w}_{0} \nu_{\mathcal{P}^{\ell}},
$$

for all $s \in\left[-2+S_{\varepsilon_{\ell}} / 8, S_{\varepsilon_{\ell}} / 8\right]$. Let $\eta(s)$ be a smooth cutoff function equal to 1 for $s \leq-3 / 2$ and vanishing for $s \geq-1$. Then, for all $s \in[-2+$ $\left.S_{\varepsilon} / 8, S_{\varepsilon} / 8\right]$, the vector $\tilde{\nu}(s, \theta)$ is defined to be the unit normal to the surface parameterized by

$$
(s, \theta) \longrightarrow \mathcal{R}_{\left(t^{\ell}, r^{\ell}\right)}\left(\mathbf{x}_{\mathcal{P}_{\ell}}+\eta\left(s+S_{\varepsilon_{\ell}} / 8\right) \hat{w}_{0} \nu_{\mathcal{P}_{\ell}}\right),
$$

As desired, $\tilde{\nu}$ is still the unit normal to $\tilde{\Sigma}_{\varepsilon, \mathcal{P}}$ when $s \leq-3 / 2-S_{\varepsilon_{\ell}} / 8$, and equals $\nu_{\mathcal{P} \ell}(s, \theta)$ when $s \in\left[-1+S_{\varepsilon_{\ell}} / 8, S_{\varepsilon_{\ell}} / 8\right]$.

Any surface near to $\tilde{\Sigma}_{\varepsilon, \mathcal{P}}$ may be parameterized by

$$
\tilde{\Sigma}_{\varepsilon, \mathcal{P}} \ni p \longrightarrow p+w(p) \tilde{\nu}(p)
$$


for some scalar valued function $w$. We need to consider the equation which $w$ must satisfy in order for this surface to have constant mean curvature one, which we shall do in a slightly more general context.

Let $S$ be a regular orientable surface, with unit normal vector field $\nu$. Suppose that $\bar{\nu}$ is another unit vector field along $S$ which is nowhere tangential. By the inverse function theorem, for any $p_{0} \in S$ there are neighbourhoods $\mathcal{U}$ and $\mathcal{V}$ near $\left(p_{0}, 0\right)$ in $S \times \mathbb{R}$ and a diffeomorphism $(\phi(p, s), \psi(p, s))$ from $\mathcal{U}$ to $\mathcal{V}$ such that

$$
p+s \nu(p)=\phi(p, s)+\psi(p, s) \bar{\nu}(\phi(p, s)) .
$$

Here $\phi(p, 0)=p$ and $\psi(p, 0)=0$. To determine the first order Taylor series of these functions in $s$, differentiate (9.6) with respect to $s$ and set $s=0$. This gives

$$
\nu(p)=\partial_{s} \phi(p, 0)+\partial_{s} \psi(p, 0) \bar{\nu}(p)
$$

and so, taking the normal component of this, we get

$$
1=\partial_{s} \psi(p, 0) \nu(p) \cdot \bar{\nu}(p), \quad \text { or } \quad \partial_{s} \psi(p, 0)=1 /(\nu(p) \cdot \bar{\nu}(p)) .
$$

Hence

$$
\psi(p, s)=\frac{s}{\nu(p) \cdot \bar{\nu}(p)}+O\left(s^{2}\right)
$$

On the other hand, taking the tangential component and using this expansion of $\psi$ yields

$$
0=\partial_{s} \phi(p, 0)+\frac{s}{\nu(p) \cdot \bar{\nu}(p)} \bar{\nu}_{t}(p)
$$

where $\bar{\nu}_{t}(p)$ is the tangential component of $\bar{\nu}$. Thus

$$
\phi(p, s)=p-\frac{s}{\nu(p) \cdot \bar{\nu}(p)} \bar{\nu}_{t}(p)+O\left(s^{2}\right) .
$$

Next, any surface which is $\mathcal{C}^{2}$ close to $S$ can be parameterized either as a normal graph of some function $w$ over $S$, using the vector field $\nu$, or as a graph of a different function $\bar{w}$ using the vector field $\bar{\nu}$. These functions are related by

$$
p+w(p) \nu(p)=\bar{p}+\bar{w}(\bar{p}) \bar{\nu}(\bar{p})=\phi(p, w(p))+\psi(p, w(p)) \bar{\nu}(\phi(p, w(p)) .
$$

Using the expansions above, we see that $\bar{w}(p)=w(p) /(\nu(p) \cdot \bar{\nu}(p))+O\left(\|w\|^{2}\right)$. 
The mean curvature operators on these two functions, which we call $H_{\nu, w}$ and $H_{\bar{\nu}, \bar{w}}$, respectively, are related by

$$
H_{\bar{\nu}, \bar{w}}(\bar{p})=H_{\nu, w}(p) .
$$

Differentiating this with respect to $\bar{w}$ and setting $\bar{w}=0$, we get

$$
D_{\bar{w}} H_{\bar{\nu}, 0}(u)=D_{w} H_{\nu, 0}((\bar{\nu} \cdot \nu) u)+\left(\nabla H_{\nu, 0} \cdot \bar{\nu}_{t}\right) u
$$

for any scalar function $u$. In the special case where the surface $S$ has constant mean curvature, this reduces to

$$
\bar{L} u:=D_{\bar{w}} H_{\bar{\nu}, 0}(u)=D_{w} H_{\nu, 0}((\bar{\nu} \cdot \nu) u):=L((\bar{\nu} \cdot \nu) u) .
$$

We apply the previous computation to the present situation. Denote by $\tilde{L}_{\varepsilon, \mathcal{P}}$ the linearized mean curvature operator about $\tilde{\Sigma}_{\varepsilon, \mathcal{P}}$. Away from $\partial \tilde{\Sigma}_{\varepsilon, \mathcal{P}}$, we have

$$
\tilde{L}_{\varepsilon, \mathcal{P}}=\frac{1}{\varepsilon^{2}} L
$$

where $L=\Delta_{\Sigma}+\left|A_{\Sigma}\right|^{2}$ is the operator we have studied in detail. Near $\partial \tilde{\Sigma}_{\varepsilon, \mathcal{P}}$ the structure of $\tilde{L}_{\varepsilon, \mathcal{P}}$ is described by the next result, the proof of which follows from the expansions given in Proposition 29.

Lemma 1. In $\varepsilon E_{\ell}$, we can write

$$
\tilde{L}_{\varepsilon, \mathcal{P}}=\frac{1}{\varepsilon^{2}} L+\hat{L}_{\varepsilon, \mathcal{P}}
$$

where $\hat{L}_{\varepsilon, \mathcal{P}}$ is a second order linear differential operator whose coefficients are supported in $\left[-2+S_{\varepsilon_{\ell}} / 8, S_{\varepsilon_{\ell}} / 8\right] \times S^{1}$ and are bounded by $\frac{1}{\varepsilon^{2} e^{2 s}} \varepsilon^{\kappa-1}$.

Also, following from the same ideas as in $\S 7.1$ is the simpler

Lemma 2. In $\varepsilon E_{\ell}$, the difference

$$
\frac{1}{\varepsilon^{2}} L-\frac{1}{\varepsilon_{\ell}^{2} \cosh ^{2} s}\left(\partial_{s s}^{2}+\partial_{\theta \theta}^{2}\right)
$$

is a second order linear differential operator, the coefficients of which are bounded by a constant times $\varepsilon^{-2} e^{-4 s}$ in $\left[s_{\ell}, S_{\varepsilon_{\ell}} / 8\right]$. 
The proofs of both of these results are left to the reader.

From these lemmas, we can immediately generalize Proposition 21 to the deformed Jacobi operators on the surfaces $\tilde{\Sigma}_{\varepsilon, \mathcal{P}}$.

Proposition 30. Fix $\mu$ with $\mu \in(1,2)$. Then there exists an $\varepsilon_{0}>0$, depending only on $\mu$, and whenever $\varepsilon \in\left(0, \varepsilon_{0}\right)$, there exist linear maps

$$
\begin{aligned}
\tilde{G}_{\varepsilon, \mathcal{P}}: \mathcal{C}_{\mu-2}^{0, \alpha}\left(\tilde{\Sigma}_{\varepsilon, \mathcal{P}}\right) & \longrightarrow \mathcal{C}_{\mu}^{2, \alpha}\left(\tilde{\Sigma}_{\varepsilon, \mathcal{P}}\right), \\
\varepsilon^{\mu / 4} \tilde{P}_{\varepsilon, \mathcal{P}}: \Pi^{\prime \prime}\left(\mathcal{C}^{2, \alpha}\left(S^{1}\right)\right)^{k} & \longrightarrow \mathcal{C}_{\mu}^{2, \alpha}\left(\tilde{\Sigma}_{\varepsilon, \mathcal{P}}\right),
\end{aligned}
$$

which are bounded uniformly as $\varepsilon \rightarrow 0$ such that, for all $f \in \mathcal{C}_{\mu-2}^{0, \alpha}\left(\tilde{\Sigma}_{\varepsilon}\right)$ and $\phi^{\prime \prime}=\left(\phi_{1}^{\prime \prime}, \ldots, \phi_{k}^{\prime \prime}\right) \in \Pi^{\prime \prime}\left(\mathcal{C}^{2, \alpha}\left(S^{1}\right)\right)^{k}$, the function $w=\tilde{G}_{\varepsilon, \mathcal{P}}(f)+\tilde{P}_{\varepsilon, \mathcal{P}}\left(\phi^{\prime \prime}\right)$ is a solution of the problem

$$
\left\{\begin{aligned}
\tilde{L}_{\varepsilon, \mathcal{P}} w & =\frac{1}{\varepsilon^{2}} f & \text { in } \quad \tilde{\Sigma}_{\varepsilon, \mathcal{P}} \\
\Pi^{\prime \prime} w & =\phi^{\prime \prime} & \text { on } \quad \partial \tilde{\Sigma}_{\varepsilon, \mathcal{P}}
\end{aligned}\right.
$$

Following the results of section $\S 4.3$, we also prove

Corollary 7. Fix $\mu \in(1,2)$. Then there exists a constant $c>0$ and an $\varepsilon_{0}>0$, depending only on $\mu$, such that for $\varepsilon \in\left(0, \varepsilon_{0}\right)$, we have

$$
\left\|\left(\tilde{P}_{\varepsilon, \mathcal{P}}-\tilde{P}_{0}\right)\left(\phi^{\prime \prime}\right)\right\|_{2, \alpha, \mu} \leq c \varepsilon^{\mu / 4}\left(\varepsilon^{\kappa-1}+\varepsilon^{(2-\mu) / 4}\right)\|\phi\|_{2, \alpha} .
$$

Here, if $\phi^{\prime \prime}=\left(\phi_{1}, \ldots, \phi_{k}\right) \in \Pi^{\prime \prime}\left(\mathcal{C}^{2, \alpha}\left(S^{1}\right)\right)^{k}$, the function $\tilde{P}_{0}\left(\phi^{\prime \prime}\right)=\tilde{w}_{0}$ is defined to be equal to $\eta\left(s-s_{\ell}\right) \tilde{w}_{\ell}$ on each end $\varepsilon E_{\ell}$ and 0 elsewhere, where $\eta$ is some cutoff function equal to 0 for. $s<0$ and equal to 1 for $s>1$ and where $\tilde{w}_{\ell}$ is the unique solution, in $\left(\mathcal{C}_{2}^{2, \alpha}\left(\left(-\infty, S_{\varepsilon_{\ell}} / 8\right] \times S^{1}\right)\right)^{k}$, of the problem

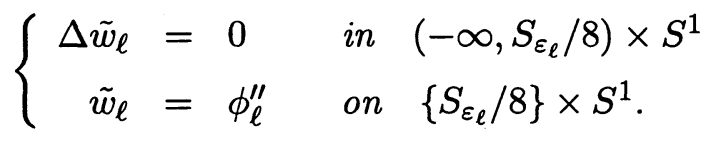

Proof. We start by solving, for each $\ell$,

$$
\left\{\begin{array}{llll}
\Delta \tilde{w}_{\ell}=0 & \text { in } & \left(-\infty, S_{\varepsilon_{\ell}} / 8\right) \times S^{1} \\
\tilde{w}_{\ell}=\phi_{\ell}^{\prime \prime} & \text { on } & \left\{S_{\varepsilon_{\ell}} / 8\right\} \times S^{1}
\end{array}\right.
$$


There is a unique solution of this equation, which is in $\mathcal{C}_{2}^{2, \alpha}\left(\left(-\infty, S_{\varepsilon_{\ell}} / 8\right] \times\right.$ $\left.S^{1}\right)$, and satisfies

$$
\left\|\tilde{w}_{\ell}\right\|_{2, \alpha, 2} \leq c \varepsilon^{1 / 2}\left\|\phi^{\prime \prime}\right\|_{2, \alpha} .
$$

Now truncate these solutions at $s=s_{\ell}$; this allows one to define $\tilde{w}_{0}$ globally on $\tilde{\Sigma}_{\varepsilon, \mathcal{P}}$ by setting it equal to 0 elsewhere. From Lemmas 1 and 2 if follows that on each end $\varepsilon E_{\ell}$, the difference

$$
\tilde{L}_{\varepsilon, \mathcal{P}}-\frac{1}{\varepsilon_{\ell}^{2} \cosh ^{2} s}\left(\partial_{s s}^{2}+\partial_{\theta \theta}^{2}\right),
$$

is a second order linear differential operator whose coefficients are sums of terms which are either bounded by a constant times $\varepsilon^{-2} e^{-4 s}$ or are supported in $\left[-2+S_{\varepsilon_{\ell}} / 8, S_{\varepsilon_{\ell}} / 8\right]$ and bounded by a constant times $\varepsilon^{-2} e^{-2 s} \varepsilon^{\kappa-1}$. Using this, we see that

$$
\left\|\tilde{L}_{\varepsilon, \mathcal{P}} \tilde{w}_{0}\right\|_{0, \alpha, \mu} \leq c\left(\varepsilon^{1 / 2}+\varepsilon^{\kappa-1+\mu / 4}\right)\left\|\phi^{\prime \prime}\right\|_{2, \alpha} .
$$

The result then follows from Proposition 30.

\section{CMC surfaces near to the truncated $k$-noids.}

Just as we already did for Delaunay surfaces, we would like to analyze the family of surfaces which are close to each $\tilde{\Sigma}_{\varepsilon, \mathcal{P}}$ and which have constant mean curvature 1 . To this end, as in (5.3), we expand the mean curvature operator to see that, for any $\phi \in \mathcal{C}^{2, \alpha}\left(\partial \tilde{\Sigma}_{\varepsilon, \mathcal{P}}\right)$, our problem reduces to solve the following boundary value problem

$$
\left\{\begin{array}{lll}
\tilde{L}_{\varepsilon, \mathcal{P}} w=1+\tilde{\mathcal{Q}}(w) & \text { in } & \tilde{\Sigma}_{\varepsilon, \mathcal{P}} \\
\Pi^{\prime \prime}(w)=\phi^{\prime \prime} & \text { on } & \partial \tilde{\Sigma}_{\varepsilon, \mathcal{P}}
\end{array}\right.
$$

Here

$$
\tilde{\mathcal{Q}}(w)=\frac{1}{\varepsilon e^{s}} \tilde{Q}\left(\frac{w}{\varepsilon e^{s}}, \frac{\nabla w}{\varepsilon e^{s}}, \frac{\nabla^{2} w}{\varepsilon e^{s}}\right),
$$

in each end $\tilde{E}_{\varepsilon}$, collects all the terms of order higher than one in $w$. The function $\tilde{\mathcal{Q}}$ has partial derivatives which are uniformly bounded. Denote by $\tilde{w}_{\varepsilon}$ the solution of

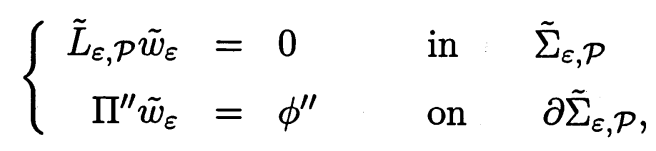


which is given by Proposition 30. By the same Proposition, we can also solve

$$
\left\{\begin{array}{rlll}
\tilde{L}_{\varepsilon, \mathcal{P}} \tilde{w}_{1}=1 & \text { in } & \tilde{\Sigma}_{\varepsilon, \mathcal{P}} \\
\Pi^{\prime \prime} \tilde{w}_{1}=0 & \text { on } & \partial \tilde{\Sigma}_{\varepsilon, \mathcal{P}}
\end{array}\right.
$$

We find that

$$
\left\|\tilde{w}_{1}\right\|_{2, \alpha, \mu} \leq c \varepsilon^{3 / 2+\mu / 4} .
$$

Setting $w=\tilde{w}_{\varepsilon}+\tilde{w}_{1}+v$, then it remains to solve

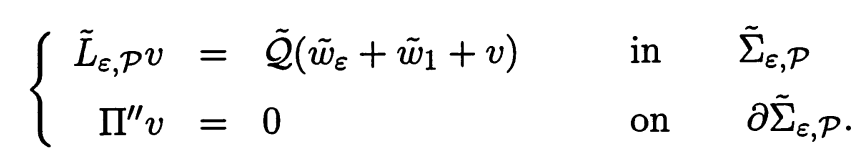

It is sufficient to find a fixed point of the mapping

$$
\tilde{\mathcal{K}}(v):=\tilde{G}_{\varepsilon, \mathcal{P}} \tilde{\mathcal{Q}}\left(\tilde{w}_{\varepsilon}+\tilde{w}_{1}+v\right),
$$

when $\varepsilon$ is sufficiently small.

Proposition 31. There exist constants $c_{0}, c_{1}>0$ such that if $\left\|\phi^{\prime \prime}\right\|_{2, \alpha} \leq$ $c_{0} \varepsilon^{3 / 4}$, then

$$
\left\|\tilde{G}_{\varepsilon}\left(\tilde{\mathcal{Q}}\left(\tilde{w}_{\varepsilon}+\tilde{w}_{1}\right)\right)\right\|_{2, \alpha, \mu} \leq \frac{c_{1}}{2} \varepsilon^{(\mu-3) / 4}\left(\left\|\phi^{\prime \prime}\right\|_{2, \alpha}^{2}+\varepsilon^{3}\right),
$$

and

$$
\left\|\tilde{G}_{\varepsilon}\left(\tilde{\mathcal{Q}}\left(\tilde{w}_{\varepsilon}+\tilde{w}_{1}+v_{2}\right)-\tilde{\mathcal{Q}}\left(\tilde{w}_{\varepsilon}+\tilde{w}_{1}+v_{1}\right)\right)\right\|_{2, \alpha, \mu} \leq \frac{1}{2}\left\|v_{2}-v_{1}\right\|_{2, \alpha, \mu},
$$

for all $v_{1}, v_{2}$ in $\tilde{B}_{c_{1}}:=\left\{v:\|v\|_{2, \alpha, \mu} \leq c_{1} \varepsilon^{(\mu-3) / 4}\left(\left\|\phi^{\prime \prime}\right\|_{2, \alpha}^{2}+\varepsilon^{3}\right)\right\}$. Thus, $\tilde{\mathcal{K}}$ is a contraction mapping on the ball $\tilde{B}_{c_{1}}$ into itself, and therefore has a unique fixed point $v$ in this ball.

Proof. We use that in each end $\varepsilon E_{\ell}$

$$
\left\|\tilde{w}_{\varepsilon}\right\|_{2, \alpha,[s, s+1]} \leq c e^{\mu\left(s-S_{\varepsilon_{\ell}} / 8\right)}\left\|\phi^{\prime \prime}\right\|_{2, \alpha},
$$

and also that

$$
\left\|\tilde{w}_{1}\right\|_{2, \alpha,[s, s+1]} \leq c \varepsilon^{3 / 2} e^{\mu\left(s-S_{\varepsilon_{\ell}} / 8\right)}
$$


These estimates imply that on the end $\varepsilon E_{\ell}$, for $s \in\left[s_{\ell}, S_{\varepsilon_{\ell}} / 8\right]$, we have

$$
e^{-\mu s}\left\|\tilde{\mathcal{Q}}\left(\tilde{w}_{\varepsilon}+\tilde{w}_{1}\right)\right\|_{0, \alpha,[s, s+1]} \leq c\left(\left\|\phi^{\prime \prime}\right\|_{2, \alpha}^{2}+\varepsilon^{3}\right) \varepsilon^{(\mu-3) / 4} .
$$

On the other hand, on the compact piece, we simply have

$$
\left\|\tilde{\mathcal{Q}}\left(\tilde{w}_{\varepsilon}+\tilde{w}_{1}\right)\right\|_{0, \alpha} \leq c\left(\left\|\phi^{\prime \prime}\right\|_{2, \alpha}^{2}+\varepsilon^{3}\right) \varepsilon^{(\mu-2) / 2} .
$$

The constant $c_{1}$ is then taken to be equal to twice the sum of the constant which appear in the last two estimates. The other estimate follows in the same way, and the proof is complete.

As in section $\S 4.3$ we finally obtain

Corollary 8. There exists a constant $c_{0}>0$ and an $\varepsilon_{0}>0$ such that, for all $\varepsilon \in\left(0, \varepsilon_{0}\right)$ and for any $\phi^{\prime \prime} \in \Pi^{\prime \prime}\left(\mathcal{C}^{2, \alpha}\left(S^{1}\right)\right)^{k}$ with $\left\|\phi^{\prime \prime}\right\|_{2, \alpha} \leq c_{0} \varepsilon^{3 / 4}$, the problem (10.1) has a unique solution $w$. The mapping

$$
\Pi^{\prime \prime}\left(\mathcal{C}^{2, \alpha}\left(S^{1}\right)\right)^{k} \ni \phi^{\prime \prime} \longrightarrow w \in \mathcal{C}_{\mu}^{2, \alpha}\left(\tilde{\Sigma}_{\varepsilon, \mathcal{P}}\right),
$$

is continuous and the solution $w$ satisfies the estimates

$$
\|w\|_{2, \alpha, \mu} \leq c \varepsilon^{\mu / 4}\left(\varepsilon^{3 / 2}+\left\|\phi^{\prime \prime}\right\|_{2, \alpha}+\varepsilon^{-3 / 4}\left\|\phi^{\prime \prime}\right\|_{2, \alpha}^{2}\right)
$$

and

$$
\begin{aligned}
& \left\|\left(w-\Pi^{\prime \prime} w\right)\left(S_{\varepsilon_{\ell}} / 8, \cdot\right)\right\|_{2, \alpha}+\left\|\partial_{s}\left(w-\Pi^{\prime \prime} w\right)\left(S_{\varepsilon_{\ell}} / 8, \cdot\right)\right\|_{1, \alpha} \\
& \quad \leq c\left(\varepsilon^{3 / 2}+\left(\varepsilon^{\kappa-1}+\varepsilon^{(2-\mu) / 4}\right)\left\|\phi^{\prime \prime}\right\|_{2, \alpha}+\varepsilon^{-3 / 4}\left\|\phi^{\prime \prime}\right\|_{2, \alpha}^{2}\right) .
\end{aligned}
$$

Finally, if $\tilde{w}_{0}=\tilde{P}_{0}\left(\phi^{\prime \prime}\right) \in \mathcal{C}_{\mu}^{2, \alpha}\left(\tilde{\Sigma}_{\varepsilon, \mathcal{P}}\right)$ as in Corollary 7 , then

$$
\left\|w-\tilde{w}_{0}\right\|_{2, \alpha, \mu} \leq c \varepsilon^{\mu / 4}\left(\varepsilon^{3 / 2}+\left(\varepsilon^{\kappa-1}+\varepsilon^{(2-\mu) / 4}\right)\left\|\phi^{\prime \prime}\right\|_{2, \alpha}+\varepsilon^{-3 / 4}\left\|\phi^{\prime \prime}\right\|_{2, \alpha}^{2}\right) .
$$

The proof is identical to that of Corollary 5 , and so we omit it.

\section{Matching the Cauchy data.}

Denote by $\mathcal{U}:=\mathbb{R}^{2 k} \times \mathbb{R}^{2 k} \times \mathbb{R}^{k} \times \mathbb{R}^{k}$ the set of parameters which is endowed with the norm defined in (9.4). We have now established that, given any 
set of parameters $\mathcal{P}=(\tilde{t}, \tilde{r}, \tilde{d}, \tilde{\delta}) \in \mathcal{U}$ satisfying $\|\mathcal{P}\| \leq \varepsilon^{5 / 4}$, and for any $\phi^{\prime \prime} \in \Pi^{\prime \prime}\left(\mathcal{C}^{2, \alpha}\left(S^{1}\right)\right)^{k}$ such that $\left\|\phi^{\prime \prime}\right\|_{2, \alpha} \leq \varepsilon^{5 / 4}$, we can solve the equations

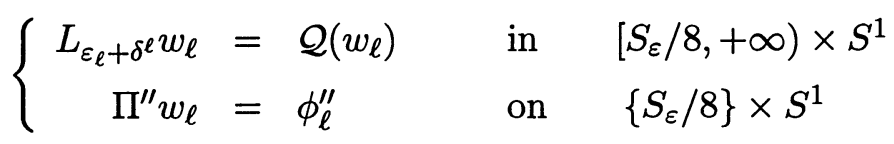

and

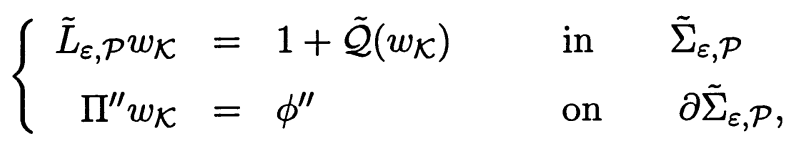

when $\varepsilon$ is sufficiently small. Thus we may define the mappings

$$
\begin{gathered}
\mathcal{S}_{\varepsilon}: \Pi^{\prime \prime}\left(\mathcal{C}^{2, \alpha}\left(S^{1}\right)\right)^{k} \ni \phi^{\prime \prime} \longrightarrow\left[\left(w_{1}, \ldots, w_{k}\right)_{\mid s=S_{\varepsilon_{\ell}} / 8},\left(\partial_{s} w_{1}, \ldots, \partial_{s} w_{k}\right)_{\mid s=S_{\varepsilon_{\ell}} / 8}\right] \\
\in\left(\mathcal{C}^{2, \alpha}\left(S^{1}\right)\right)^{k} \times\left(\mathcal{C}^{1, \alpha}\left(S^{1}\right)\right)^{k},
\end{gathered}
$$

and

$$
\begin{aligned}
& \mathcal{T}_{\varepsilon}: \mathcal{U} \times \Pi^{\prime \prime}\left(\mathcal{C}^{2, \alpha}\left(S^{1}\right)\right)^{k} \ni \phi^{\prime \prime} \\
& {\left[\left(\left.\left(\hat{w}_{0}+w_{\mathcal{K}}\right)\right|_{\varepsilon E_{1}}, \ldots,\left.\left(\hat{w}_{0}+w_{\mathcal{K}}\right)\right|_{\varepsilon E_{k}}\right)_{\mid s=S_{\varepsilon_{\ell}} / 8},\right.} \\
&\left.\left(\left.\partial_{s}\left(\hat{w}_{0}+w_{\mathcal{K}}\right)\right|_{\varepsilon E_{1}}, \ldots,\left.\partial_{s}\left(\hat{w}_{0}+w_{\mathcal{K}}\right)\right|_{\varepsilon E_{k}}\right)_{\mid s=S_{\varepsilon_{\ell}} / 8}\right] \\
& \quad \in\left(\mathcal{C}^{2, \alpha}\left(S^{1}\right)\right)^{k} \times\left(\mathcal{C}^{1, \alpha}\left(S^{1}\right)\right)^{k}
\end{aligned}
$$

where $\hat{w}_{0}$ is defined in Proposition 29. These would be the Cauchy data mappings for the two nonlinear problems (11.1) and (11.2).

Finally, we extend $\mathcal{S}_{\varepsilon}$ trivialy to $\mathcal{U} \times \Pi^{\prime \prime}\left(\mathcal{C}^{2, \alpha}\left(S^{1}\right)\right)^{k}$ and we set

$$
C_{\varepsilon}:=\mathcal{S}_{\varepsilon}-\mathcal{T}_{\varepsilon}
$$

By construction $C_{\varepsilon}$ has range in a subset of $\left(\mathcal{C}^{2, \alpha}\left(S^{1}\right)\right)^{k} \times\left(\mathcal{C}^{1, \alpha}\left(S^{1}\right)\right)^{k}$, namely

$$
\text { range } C_{\varepsilon} \subset\left(\operatorname{Span}\left\{1, e^{i \theta}, e^{-i \theta}\right\}\right)^{k} \times\left(\mathcal{C}^{1, \alpha}\left(S^{1}\right)\right)^{k} .
$$

It follows from Corollary 5 and Corollary 8 that, for all $\varepsilon>0$ small enough, these mappings are well defined from the ball of radius $\varepsilon^{5 / 4}$ in $\mathcal{U} \times\left(\mathcal{C}^{2, \alpha}\left(S^{1}\right)\right)^{k}$.

Proposition 32. For some $\varepsilon_{0}>0$ and every $\varepsilon \in\left(0, \varepsilon_{0}\right)$, there exists a $\left(\mathcal{P}, \phi^{\prime \prime}\right) \in \mathcal{U} \times\left(\mathcal{C}^{1, \alpha}\left(S^{1}\right)\right)^{k}$ which solves $C_{\varepsilon}\left(\mathcal{P}, \phi^{\prime \prime}\right)=0$. 
This produces the desired CMC surface with $k$ Delaunay ends.

Proof. We define mappings

$$
\begin{aligned}
\mathcal{S}_{0}: \Pi^{\prime \prime}\left(\mathcal{C}^{2, \alpha}\left(S^{1}\right)\right)^{k} \ni \phi^{\prime \prime} & \longrightarrow \\
& {\left[\left(w_{1}, \ldots, w_{k}\right)_{\mid s=S_{\varepsilon_{\ell}} / 8},\left(\partial_{s} w_{1}, \ldots, \partial_{s} w_{k}\right)_{\mid s=S_{\varepsilon_{\ell}} / 8}\right] } \\
& \in\left(\mathcal{C}^{2, \alpha}\left(S^{1}\right)\right)^{k} \times\left(\mathcal{C}^{1, \alpha}\left(S^{1}\right)\right)^{k},
\end{aligned}
$$

where this time $w_{\ell}=P_{0}\left(\phi_{\ell}^{\prime \prime}\right)$ as in Corollary 4 and

$$
\begin{gathered}
\mathcal{T}_{0}: \mathcal{U} \times \Pi^{\prime \prime}\left(\mathcal{C}^{2, \alpha}\left(S^{1}\right)\right)^{k} \ni \phi^{\prime \prime} \longrightarrow \\
{\left[\left(\left.\left(\check{w}_{0}+\tilde{w}_{0}\right)\right|_{\varepsilon E_{1}}, \ldots,\left.\left(\check{w}_{0}+\tilde{w}_{0}\right)\right|_{\varepsilon E_{k}}\right)_{\mid s=S_{\varepsilon_{\ell} / 8}},\right.} \\
\left.\left(\left.\partial_{s}\left(\check{w}_{0}+\tilde{w}_{0}\right)\right|_{\varepsilon E_{1}}, \ldots,\left.\partial_{s}\left(\check{w}_{0}+\tilde{w}_{0}\right)\right|_{\varepsilon E_{k}}\right)_{\mid s=S_{\varepsilon_{\ell}} / 8}\right] \\
\quad \in\left(\mathcal{C}^{2, \alpha}\left(S^{1}\right)\right)^{k} \times\left(\mathcal{C}^{1, \alpha}\left(S^{1}\right)\right)^{k},
\end{gathered}
$$

where $\tilde{w}_{0}=\tilde{P}_{0}\left(\phi^{\prime \prime}\right)$ as in Corollary 7 and where we have set

$$
\begin{aligned}
\left.\check{w}_{0}\right|_{\varepsilon E_{\ell}}= & -\frac{1}{\cosh s}\left(t_{1}^{\ell} \cos \theta+t_{2}^{\ell} \sin \theta\right) \\
& -\left(r_{1}^{\ell} \cos \theta+r_{2}^{\ell} \sin \theta\right) \varepsilon_{\ell} \cosh s+d^{\ell}+\delta^{\ell}\left(s^{\prime}-1\right) .
\end{aligned}
$$

It is an easy exercise to check that $C_{0}$ is an isomorphism from $\mathcal{U} \times$ $\Pi^{\prime \prime}\left(\mathcal{C}^{2, \alpha}\left(S^{1}\right)\right)^{k}$ into $\left(\operatorname{Span}\left\{1, e^{i \theta}, e^{-i \theta}\right\}\right)^{k} \times\left(\mathcal{C}^{1, \alpha}\left(S^{1}\right)\right)^{k}$ (with the norm induced by the norm of $\left.\left(\mathcal{C}^{2, \alpha}\left(S^{1}\right)\right)^{k} \times\left(\mathcal{C}^{1, \alpha}\left(S^{1}\right)\right)^{k}\right)$, whose inverse is bounded independently of $\varepsilon$. In particular, the equation $C_{0}\left(\mathcal{P}, \phi^{\prime \prime}\right)=0$ has the unique solution $(0,0)$.

By Corollaries 5, 8 and Proposition 29, we may estimate

$$
\left\|\left(C_{\varepsilon}-C_{0}\right)\left(\mathcal{P}, \phi^{\prime \prime}\right)\right\|_{\left(\mathcal{C}^{2, \alpha}\left(S^{1}\right)\right)^{k} \times\left(\mathcal{C}^{1, \alpha}\left(S^{1}\right)\right)^{k}} \leq c \varepsilon^{(7-\mu) / 4} .
$$

In order to solve $C_{\varepsilon}\left(\mathcal{P}, \phi^{\prime \prime}\right)=0$, it is enough to find a fixed point for the nonlinear mapping

$$
F_{\varepsilon}\left(\mathcal{P}, \phi^{\prime \prime}\right):=C_{0}^{-1}\left(\left(C_{\varepsilon}-C_{0}\right)\left(\mathcal{P}, \phi^{\prime \prime}\right)\right) .
$$

In order to prove that such a fixed point exists we would like to use LeraySchauder degree theory in the ball of radius $\varepsilon^{5 / 4}$ in $\mathcal{U} \times \Pi^{\prime \prime}\left(\mathcal{C}^{2, \alpha}\left(S^{1}\right)\right)^{k}$. Continuity of $F_{\varepsilon}$ follows readily from the construction of $\mathcal{S}_{\varepsilon}$ and $\mathcal{T}_{\varepsilon}$. However, 
$F_{\varepsilon}$ fails to be compact. To overcome this last difficulty, we define a family of smoothing mappings

$$
\mathcal{D}_{q}: \sum_{n \in \mathbb{Z}} a_{n} e^{i n \theta} \longrightarrow \sum_{n \in \mathbb{Z}}\left(1+n^{2}\right)^{q / 2} a_{n} e^{i n \theta} .
$$

When $q<0$, the mapping $\mathcal{D}_{q}$ is bounded on $\mathcal{C}^{2, \alpha}\left(S^{1}\right)$, with norm uniformly bounded as $q \rightarrow 0$. We extend this mapping to $\mathcal{U} \times \Pi^{\prime \prime}\left(\mathcal{C}^{2, \alpha}\left(S^{1}\right)\right)^{k}$ in the obvious way and for all $q<0$, we look for a solution of

$$
\left(\mathcal{P}_{q}, \phi_{q}^{\prime \prime}\right)=\mathcal{D}_{q} C_{0}^{-1}\left(\left(C_{\varepsilon}-C_{0}\right)\left(\mathcal{P}_{q}, \phi_{q}^{\prime \prime}\right)\right) .
$$

This operator is compact and for $\varepsilon$ suficiently small, maps the ball of radius $\varepsilon^{5 / 4}$ to itself, because all the terms in $F_{\varepsilon}$ decay faster (in $\varepsilon$ ) than $\varepsilon^{5 / 4}$. Hence it has a fixed point when $\varepsilon$ is small enough. Finally, the fixed points are bounded uniformly in $q$, so for any fixed $\alpha^{\prime}<\alpha$ we may extract a sequence $q_{j} \rightarrow 0$ such that $\left(\mathcal{A}_{q_{j}}, \phi_{q_{j}}^{\prime \prime}\right)$ converges in $\mathcal{U} \times \Pi^{\prime \prime}\left(\mathcal{C}^{2, \alpha^{\prime}}\left(S_{1}\right)\right)^{k}$. $\mathbf{C}_{\varepsilon}$ vanishes at the limit of this sequence. This completes the proof.

\section{The nondegeneracy of the solutions.}

We now show that for $\varepsilon$ sufficiently small, the solutions we have constructed above are nondegenerate in the sense defined in [9]. This condition ensures the smoothness of the moduli spaces $\mathcal{M}_{g, k}$ near $\Sigma_{\varepsilon}$. We begin by recalling this notion of nondegeneracy.

Definition 3. The constant mean curvature surface $\Sigma_{\varepsilon} \in \mathcal{M}_{g, k}$ is nondegenerate if the linearization of the mean curvature operator about $\Sigma_{\varepsilon}$ is injective on the function space $\mathcal{C}_{\delta}^{2, \alpha}\left(\Sigma_{\varepsilon}\right)$ for all $\delta<0$.

Here for $r \in \mathbb{N}, \alpha \in[0,1)$ and $\delta \in \mathbb{R}, \mathcal{C}_{\delta}^{r, \alpha}\left(\Sigma_{\varepsilon}\right)$ is defined to be the space of functions $\phi \in \mathcal{C}^{r, \alpha}\left(\Sigma_{\varepsilon}\right)$ which can be written on each end of $\Sigma_{\varepsilon}$ as $e^{\delta s}$ times a function $\psi$ with $\psi \in \mathcal{C}^{r, \alpha}\left(\mathbb{R}_{s}^{+} \times S_{\theta}^{1}\right)$.

First notice that it is sufficient to prove that, for $\varepsilon$ small enough, the Jacobi operator $L$ is injective on $\mathcal{C}_{\delta}^{2, \alpha}\left(\Sigma_{\varepsilon}\right)$ for some fixed $\delta \in(-2,-1)$. This is because any decaying solution of $L u=0$ must decay exponentially near the $i$ th end of $\Sigma_{\varepsilon}$ at least like $e^{-\gamma_{2}\left(\varepsilon_{i}\right) s}$, and by Proposition 20, when $\varepsilon$ is sufficiently small, $2-\gamma_{2}\left(\varepsilon_{i}\right)$ is as small as desired, so that $u \in \mathcal{C}_{\delta}^{2, \alpha}\left(\Sigma_{\varepsilon}\right)$.

The proof is by contradiction. Fix $\delta \in(-2,-1)$ and assume that for some sequence of $\varepsilon_{k}$ tending to 0 , the Jacobi operator

$$
\mathcal{L}_{k}=\Delta_{\Sigma_{\varepsilon_{k}}}+\left|\mathbf{A}_{\Sigma_{\varepsilon_{k}}}\right|^{2}
$$


on $\Sigma_{\varepsilon_{k}}$ is not injective on $\mathcal{C}_{\delta}^{2, \alpha}\left(\Sigma_{\varepsilon_{k}}\right)$. Then there exists some $w_{k} \in \mathcal{C}_{\delta}^{2, \alpha}\left(\Sigma_{\varepsilon_{k}}\right)$ such that $\mathcal{L}_{k} w_{k}=0$ and $w_{k} \neq 0$.

First normalize $w_{k}$, multiplying it by a suitable constant, so that $\left\|w_{k}\right\|_{0,0, \delta}=1$. Choose a point $y_{k} \in \Sigma_{\varepsilon_{k}}$ where the above norm is achieved. Suppose first that some subsequence of the $y_{k} / \varepsilon_{k}$ converges to a point $y_{0} \in \Sigma_{0}$. Then we can extract a subsequence of the $w_{k}$ which converge on every compact of $\Sigma_{0}$ to a limiting function $w$ globally defined on $\Sigma_{0} ; w$ must be nontrivial since we also have $\|w\|_{0,0, \delta}=1$. Furthermore, $L_{\Sigma_{0}} w=0$. Since we have asssumed that $\Sigma_{0}$ is nondegenerate, we have obtained a contradiction.

If, on the other hand, some subsequence of the $y_{k}$ satisfies

$$
\lim _{k \rightarrow+\infty}\left|y_{k} / \varepsilon_{k}\right|=+\infty
$$

then, this implies that, at least for a subsequence, the points $y_{k}$ are always in the same end, say the $i$ th. Therefore, we may write,

$$
y_{k}=\mathbf{x}_{\varepsilon_{k}, i}\left(s_{k}+s_{\varepsilon_{k}, i}, \theta_{k}\right),
$$

with $s_{k}$ tending to $+\infty$. By translating back by $s_{k}+s_{\varepsilon_{k}, i}$ and multiplying by a suitable constant, we find yet another sequence of solutions, which we again call $w_{k}$, attaining their maximum at $s=0$, and which solve the translated equation, which we again write as $\mathcal{L}_{k} w_{k}=0$. Here $\mathcal{L}_{k}$ is the linearized mean curvature operator relative to the parameterization given above near the ends. It is straightforward to see that the $w_{k}$ converge to a nontrivial solution $w$ of one of the following two limiting equations

$$
\partial_{s s}^{2} w+\partial_{\theta \theta}^{2} w=0
$$

or

$$
\partial_{s s}^{2} w+\partial_{\theta \theta}^{2} w+\frac{2}{\cosh ^{2}(s+\bar{s})} w=0, \quad \text { for some } \quad \bar{s} \in \mathbb{R}
$$

on $\mathbb{R} \times S^{1}$. In addition, $w$ is bounded by $e^{\delta s}$. By decomposing into eigenfrequencies we then see that necessarily $w=0$ which is the desired contradiction.

This covers all cases, so we have showed that the linearization is injective on the appropriate weighted Hölder spaces. 


\section{References.}

[1] C. Cosin and A. Ros, A Plateau problem at infinity for properly immersed minimal surfaces with finite total curvature, Preprint, 1998.

[2] C. Delaunay, Sur la surface de revolution dont la courbure moyenne est constante, J. de Mathématiques, 6 (1841), 309-320.

[3] K. Grosse-Brauckmann, New surfaces of constant mean curvature, Math. Z. 214 (1993), 527-565.

[4] K. Grosse-Brauckmann, R. Kusner and J. Sullivan. Constant mean curvature surfaces with three ends, To appear, Proc. Acad. Nat. Sci.

[5] N. Kapouleas, Complete constant mean cuvature surfaces in Euclidean three space, Ann. of Math. (2) 131 (1990), 239-330.

[6] N. Kapouleas, Complete embedded minimal surfaces of finite total curvature, J. Diff. Geom. 47 (1997), 95-169.

[7] N. Kapouleas, Constant mean curvature surfaces constructed by fusing Wente tori, Invent. Math. 119 (1995), 443-518.

[8] N. Korevaar, R. Kusner and B. Solomon, The structure of complete embedded surfaces with constant mean curvature, J. of Diff. Geometry, 30 (1989), 465503.

[9] R. Kusner, R. Mazzeo and D. Pollack, The moduli space of complete embedded constant mean curvature surfaces, Geom. Funct. Anal. 6 (1996), 120-137.

[10] R. Mazzeo and F. Pacard, Constant scalar curvature metrics with isolated singularities, Duke Math. J. 99 No. 3 (1999), 353-418.

[11] R. Mazzeo, F. Pacard and D. Pollack, Connected sums of constant mean curvature surfaces in Euclidean 3 space. To appear, J. Reine Ang. Math.

[12] R. Mazzeo and D. Pollack, Gluing and moduli for some noncompact geometric problems, in Geometric Theory of Singular Phenomena in Partial Differential Equations, Symposia Mathematica Vol XXXVIII, Cambridge Univ. Press (1998), 17-51.

[13] R. Mazzeo, D. Pollack and K. Uhlenbeck Moduli spaces of singular Yamabe metrics, J. Amer. Math. Soc. 9 (1996), 303-344.

[14] W. Meeks III, The topology and geometry of embedded surfaces of constant mean curvature, J. Differential Geometry 27 (1988), 539-552.

[15] S. Nayatani, Morse index of complete minimal surfaces, in The problem of Plateau, ed. Th. M. Rassias, World Scientific Pub. Co. (1992), 181-189. 
[16] R. Osserman, A survey of minimal surfaces Dover Publications, New York, 2nd Edition (1986).

[17] J. Perez and A. Ros, The space of properly embedded minimal surfaces with finite total curvature, Indiana Univ. Math. J. 45 (1996), 177-204.

[18] R. Schoen, The existence of weak solutions with prescribed singular behavior for a conformally invariant scalar equation Comm. Pure and Appl. Math. XLI (1988), 317-392.

[19] R. Schoen, Uniqueness, symmetry and embeddedness of minimal surfaces, J. of Diff. Geometry, 18 (1983), 791-809.

[20] H. Wente, Complete immersions of constant mean curvature, Proc. Sympos. Pure Math. 54 Amer. Math. Soc. (1993).

[21] S. D. Yang, A connected sum construction for complete minimal surfaces with finite total curvature, Preprint.

Received March 3, 1999.

STANFORD UNIVERSITY

AND

UNIVERSITÉ PARIS XII 